\title{
NUCLEARITY RELATED PROPERTIES IN OPERATOR SYSTEMS
}

\author{
ALI S. KAVRUK
}

\begin{abstract}
Some recent research on the tensor products of operator systems and ensuing nuclearity properties in this setting raised many stability problems. In this paper we examine the preservation of these nuclearity properties including exactness, local liftability and the double commutant expectation property under basic algebraic operations such as quotient, duality, coproducts and tensorial products. We show that, in the finite dimensional case, exactness and the lifting property are dual pairs, that is, an operator system $\mathcal{S}$ is exact if and only if the dual operator system $\mathcal{S}^{d}$ has the lifting property. Moreover, the lifting property is preserved under quotients by null subspaces.

Again in the finite dimensional case we prove that every operator system has the $k$-lifting property in the sense that whenever $\varphi: \mathcal{S} \rightarrow \mathcal{A} / I$ is a unital and completely positive map, where $\mathcal{A}$ is a $\mathrm{C}^{*}$-algebra and $I$ is an ideal, then $\varphi$ possess a unital $k$-positive lift on $\mathcal{A}$, for every $k$. This property provides a novel proof of a classical result of Smith and Ward on the preservation of matricial numerical ranges of an operator.

The Kirchberg conjecture naturally falls into this context. We show that the Kirchberg conjecture is equivalent to the statement that the five dimensional universal operator system generated by two contraction $\left(\mathcal{S}_{2}\right)$ has the double commutant expectation property. In addition to this we give several equivalent statements to this conjecture regarding the preservation of various nuclearity properties under basic algebraic operations.

We show that the Smith Ward problem is equivalent to the statement that every three dimensional operator system has the lifting property (or exactness). If we suppose that both the Kirchberg conjecture and the Smith Ward problem have an affirmative answer then this implies that every three dimensional operator system is $\mathrm{C}^{*}$-nuclear. We see that this property, even under most favorable conditions, seems to be hard to verify.
\end{abstract}

The study of tensor products and therefore the behavior of objects under the tensorial operations is fundamental in operator theory. Exactness, local liftability, approximation property and weak expectation are some structural properties of $\mathrm{C}^{*}$-algebras which are known to be deeply connected with the tensor product. The operator space versions and nonselfadjoint analogues of these properties have been worked out in the last decade (see 41, Sec. 15,16,17] and [3]). After being abstractly characterized by Choi and Effros, operator systems played an important role in the understanding of tensor products of $\mathrm{C}^{*}$-algebras, nuclearity, injectivity, etc. (see [28, [7], 6], e.g.). Some special tensor products of two operator systems are also used in quantum mechanics ([38, e.g.). However a systematic study of tensor products on this category along with the characterization of nuclearity properties waited till [22] and 21] (see also [17]). This series of papers raised several questions; namely, the stability of these properties under certain operations which is the main subject of the present paper. More precisely we try to illuminate the behavior of the nuclearity properties under basic algebraic constructions such as quotients, coproducts, duality, tensors etc.

We start with a brief introduction to operator systems together with their abstract characterization. We also include some special $\mathrm{C}^{*}$-covers generated by an operator system and we continue with the basic duality results in this category. In Section 2 we recall basic facts

Date: July 13, 2011

... 
on the quotient theory of operator systems. This especially allows us to utilize exactness in this category.

Section 3 includes a brief overview on the tensor products of operator systems. After giving the axiomatic definition we recall basic facts on the minimal ( $\min )$, the maximal $(\max )$, the (maximal) commuting (c), enveloping left (el) and enveloping right (er) tensor products. The set of tensor products admits a natural partial order and the primary tensor products we have considered exhibit the following relations:

$$
\min \leq e l, \text { er } \leq c \leq \max .
$$

Nuclearity forms the integral part of Section 4. Given two operator system tensor products $\alpha \leq \beta$, an operator system $\mathcal{S}$ is said to be $(\alpha, \beta)$-nuclear if $\mathcal{S} \otimes_{\alpha} \mathcal{T}=\mathcal{S} \otimes_{\beta} \mathcal{T}$ for every operator system $\mathcal{T}$. One of the main goals of [21] (see also [17]) is to characterize the nuclearity properties among the primary tensor products above which forms the following equivalences:

$(\min , \max )$-nuclearity $=$ completely positive factorization property $(\mathrm{CPFP})$,

$($ min,el)-nuclearity $=$ exactness,

(min,er)-nuclearity $=$ (operator system) local lifting property (osLLP),

$(\mathrm{el}, \mathrm{c})$-nuclearity $=$ double commutant expectation property $(\mathrm{DCEP})$,

$(\mathrm{el}, \max )$-nuclearity $=$ weak expectation property $(\mathrm{WEP})$.

We remark that WEP and DCEP coincides for $\mathrm{C}^{*}$-algebras. Also, again for $\mathrm{C}^{*}$-algebras, Kirchberg's local lifting property (LLP) and osLLP coincides. For finite dimensional operator systems we simply use the term "lifting property".

We consider Sections 1,2,3 and 4 as the basic part of the paper. Since many of the constructions in later sections are applicable to the Kirchberg conjecture we put the related discussion in Section 5. Recall that the Kirchberg conjecture is equivalent to an outstanding problem in von Neumann algebra theory, namely Connes' embedding problem, and it states that every $\mathrm{C}^{*}$-algebra that has LLP has WEP. Since these properties extend to general operator systems it is natural to approach this conjecture from an operator system perspective. In 21] it was shown that the Kirchberg conjecture has an affirmative answer if and only if every finite dimensional operator system with the lifting property has DCEP. One of our main goals in Section 5 is to obtain an even simpler form of this. Let $C^{*}\left(\mathbb{F}_{n}\right)$ represent the full $\mathrm{C}^{*}$-algebra of the free group $\mathbb{F}_{n}$ on $n$ generators (equipped with the discrete topology). We define

$$
\mathcal{S}_{n}=\operatorname{span}\left\{g_{1}, \ldots, g_{n}, e, g_{1}^{*}, \ldots, g_{n}^{*}\right\} \subset C^{*}\left(\mathbb{F}_{n}\right)
$$

where the $g_{i}$ 's are the unitary generators of $C^{*}\left(\mathbb{F}_{n}\right)$. One can consider $\mathcal{S}_{n}$ as the universal operator system generated by $n$ contractions as it is the unique operator system with the following property: Whenever $y_{1}, \ldots, y_{n}$ are contractive elements of an operator system $\mathcal{T}$ then there is a unique unital and completely positive (ucp) map $\varphi: \mathcal{S}_{n} \rightarrow \mathcal{T}$ satisfying $\varphi\left(g_{i}\right)=y_{i}$ for $i=1, \ldots, n$. As pointed out in [21], $\mathcal{S}_{n}$ has the lifting property for every $n$. One of our main results in Section 5 is the operator system analogue of Kirchberg's WEP characterization ([25], see also [41, Thm. 15.5]): A unital $\mathrm{C}^{*}$-algebra $\mathcal{A}$ has WEP is and only if $\mathcal{A} \otimes_{\min } \mathcal{S}_{2}=\mathcal{A} \otimes_{\max } \mathcal{S}_{2}$. Turning back to the Kirchberg conjecture we obtain the following five dimensional operator system variant.

Theorem 0.1. The following are equivalent:

(1) The Kirchberg conjecture has an affirmative answer.

(2) $\mathcal{S}_{2}$ has DCEP.

(3) $\mathcal{S}_{2} \otimes_{\min } \mathcal{S}_{2}=\mathcal{S}_{2} \otimes_{c} \mathcal{S}_{2}$. 
When $E$ and $F$ are Banach spaces then the natural algebraic inclusion of the minimal Banach space tensor product $E \hat{\otimes} F$ into $B\left(E^{*}, F\right)$ is an isometry. Moreover, when $E$ is finite dimensional this inclusion is bijective. A similar embedding and bijectivity are also true in the non-commutative setting, that is, the same inclusion is a complete isometry if one uses the minimal operator space tensor product and considers completely bounded maps. Since the dual of a finite dimensional operator system is again an operator system we have a similar representation of the minimal operator system tensor product. In Section 6 we give several applications of this result. In particular we show that exactness and the lifting property are dual pairs. We also show that the lifting property of a finite dimensional operator system is preserved under quotient by a null subspace, in contrast to $\mathrm{C}^{*}$-algebra ideal quotients.

In Section 7 we adapt some of the results of Ozawa and Pisier in the operator space setting to operator systems. We primarily show that $\mathbb{B}=B(H)$ and $\mathbb{K}=K(H)$, the ideal of compact operators, where $H=l^{2}$, are universal objects for the verification of exactness and the lifting property. More precisely we prove that an operator system $\mathcal{S}$ is exact if and only if the induced map

$$
\left(\mathcal{S} \hat{\otimes}_{\min } \mathbb{B}\right) /(\mathcal{S} \bar{\otimes} \mathbb{K})=\mathcal{S} \hat{\otimes}_{\min }(\mathbb{B} / \mathbb{K})
$$

is a complete order isomorphism. (Here $\hat{\otimes}_{\min }$ represents the completed minimal tensor product and $\bar{\otimes}$ is the closure of the algebraic tensor product.) Likewise a finite dimensional operator system $\mathcal{S}$ has the lifting property if and only if every ucp map $\varphi: \mathcal{S} \rightarrow \mathbb{B} / \mathbb{K}$ has a ucp lift on $\mathbb{B}$.

The amalgamated sum of two operator systems over the unit introduced in [23] (or coproduct of two operator systems in the language of [13]) seem to be another natural structure to seek the stability of several nuclearity properties. In Section 8 we first describe the coproduct of two operator systems in terms of operator system quotients and then we show that the lifting property is preserved under this operation. The stability of the double commutant expectation property, with some additional assumptions, seems to be a hard problem. We show that an affirmative answer to such a question is directly related to the Kirchberg Conjecture. More precisely if $\mathcal{S}=\operatorname{span}\left\{1, z, z^{*}\right\} \subset C(\mathbb{T})$, where $z$ is the coordinate function on the unit circle $\mathbb{T}$, then the Kirchberg conjecture is equivalent to the statement that the five dimensional operator system $\mathcal{S} \oplus_{1} \mathcal{S}$, the coproduct of $\mathcal{S}$ with itself, has the double commutant expectation property. (Note: Here $\mathcal{S}$ coincides with $\mathcal{S}_{1}$ and $\mathcal{S} \oplus_{1} \mathcal{S}$ coincides with $\mathcal{S}_{2}$.)

In [44, Xhabli introduces the $k$-minimal and $k$-maximal structure on an operator system $\mathcal{S}$. After recalling the universal properties of these constructions we studied the nuclearity within this context. In particular, we show that if an operator system is equipped with the $k$-minimal structure it has exactness and, in the finite dimensional case, the $k$-maximal structure automatically implies the lifting property. This allow us to show that every finite dimensional operator system has the $k$-lifting property, that is, if $\varphi: \mathcal{S} \rightarrow \mathcal{A} / I$ is a ucp map, where $\mathcal{A}$ is a $\mathrm{C}^{*}$-algebra and $I$ is an ideal in $\mathcal{A}$, then $\varphi$ has a unital $k$-positive lifting on $\mathcal{A}$ $($ for every $k$ ).

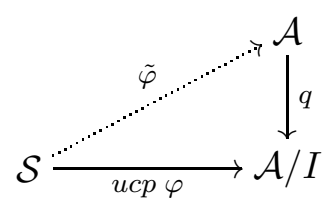


From the nuclearity point of view matrix algebras are the best understood objects: In addition to being nuclear, for an arbitrary $\mathrm{C}^{*}$-algebra, completely positive factorization property through matrix algebras is equivalent to nuclearity (see [7 e.g.). However, the quotients of the matrix algebras by some special kernels, or certain operator subsystems of the these algebras under duality raise several difficult problems. In Section 10 we first recall these quotient and duality results given in [10. We simplify some of the proofs and discuss the Kirchberg conjecture in this setting. In fact we see that this conjecture is a quotient and duality problem in the category of operator systems. We also look at the triple Kirchberg conjecture (Conjecture 10.18). The property $\mathbb{S}_{2}$, which coincides with Lance's weak expectation for $\mathrm{C}^{*}$-algebras, appear to be at the center of understanding of these conjectures.

The Smith Ward problem (SWP), which is a question regarding the preservation of matricial numerical range of an operator under compact perturbation, goes back to 1980. In Section 11 we abstractly characterize this problem. More precisely, we see that SWP is a general three dimensional operator system problem rather than a proper compact perturbation of an operator in the Calkin algebra. The following is our main result in Section 11:

Theorem 0.2. The following are equivalent:

(1) SWP has an affirmative answer.

(2) Every three dimensional operator system has the lifting property.

(3) Every three dimensional operator system is exact.

This version allows us to combine this problem with the Kirchberg conjecture (KC). In fact, if we assume both SWP and KC then this would imply that every three dimensional operator system is $\mathrm{C}^{*}$-nuclear. On the other hand the latter condition implies SWP. This lower dimensional operator system problem seems to be very hard. Even for an operator system of the form $\mathcal{S}=\operatorname{span}\left\{1, z, z^{*}\right\} \subset C(X)$, where $X$ is a compact subset of the unit disk $\{z:|z| \leq 1\}$ and $z$ is the coordinate function, we don't know whether $\mathcal{S}$ is $\mathrm{C}^{*}$-nuclear.

\section{Preliminaries}

In this section we establish the terminology and state the definitions and basic results that shall be used throughout the paper. By a *-vector space we mean a complex vector space $V$ together with a map $*: V \rightarrow V$ that is involutive (i.e. $\left(v^{*}\right)^{*}=v$ for all $v$ in $V$ ) and conjugate linear (i.e. $(\alpha v+w)^{*}=\bar{\alpha} v^{*}+w^{*}$ for all scalar $\alpha$ and $\left.v, w \in V\right)$. An element $v \in V$ is called hermitian (or selfadjoint) if $v=v^{*}$. We let $V_{h}$ denote the set of all hermitian elements of $V$. By $M_{n, k}(V)$ we mean $n \times k$ matrices whose entries are elements of $V$, that is, $M_{n, k}(V)=\left\{\left(v_{i j}\right)_{i, j}: v_{i j} \in V\right.$ for $i=1, \ldots, n$ and $\left.j=1, \ldots, m\right\}$ and we use the notation $M_{n}(V)$ for $M_{n, n}(V)$. Note that $M_{n}(V)$ is again a $*$-vector space with $\left(v_{i j}\right)^{*}=\left(v_{j i}^{*}\right)$. We let $M_{n, k}$ denote the $n \times k$ matrices with complex entries and set $M_{n}=M_{n, n}$. If $A=\left(a_{i j}\right)$ is in $M_{m, n}$ and $X=\left(v_{i j}\right)$ is in $M_{n, k}(V)$ then the multiplication $A X$ is an element of $M_{m, k}(V)$ whose $i j^{\text {th }}$ entry is equal $\Sigma_{r} a_{i r} v_{r j}$ for $i=1, \ldots, m$ and $j=1, \ldots, k$. We define a right multiplication with appropriate size of matrices in a similar way.

If $V$ is a $*$-vector space then by a matrix ordering (or a matricial order) on $V$ we mean a collection $\left\{C_{n}\right\}_{n=1}^{\infty}$ where each $C_{n}$ is a cone in $M_{n}(V)_{h}$ and the following axioms are satisfied:

(1) $C_{n}$ is strict, that is, $C_{n} \cap\left(-C_{n}\right)=\{0\}$ for every $n$.

(2) $\left\{C_{n}\right\}$ is compatible, that is, $A^{*} C_{n} A \subseteq C_{m}$ for every $A$ in $M_{n, m}$ and for every $n, m$. 
The $*$-vector space $V$ together with the matricial order structure $\left\{C_{n}\right\}$ is called a matrix ordered *-vector space. An element in $C_{n}$ is called a positive element of $M_{n}(V)$. There is a natural (partial) order structure on $M_{n}(V)_{h}$ given by $A \leq B$ if $B-A$ is in $C_{n}$. We finally remark that we often use the notation $M_{n}(V)^{+}$for $C_{n}$. Perhaps the most important examples of these spaces are $*$-closed subspaces of a $B(H)$, bounded linear operators on a Hilbert space $H$, together with the induced matricial positive cone structure. More precisely, if $V$ is such a subspace then $M_{n}(V)$ is again a $*$-closed subspace of $M_{n}(B(H))$ which can be identified with $B(H \oplus \cdots \oplus H)$, bounded operators on direct sum of $n$ copies of $H$. By using this identification we will set $C_{n}=M_{n}(V) \cap M_{n}(B(H))^{+}$, where $M_{n}(B(H))^{+}$denotes the positive elements of $M_{n}(B(H))$. It is elementary to verify that the collection $\left\{C_{n}\right\}$ forms a matrix ordering on the $*$-vector space $V$.

An element $e$ of a matrix ordered $*$-vector space $V$ is called an order unit if for every selfadjoint element $v$ of $V$ there is a positive real number $\alpha$ such that $\alpha e+v \geq 0$. Note that $e$ must be a positive element. We say that $e$ is matrix order unit if the corresponding $n \times n$ matrix given by

$$
e_{n}=\left(\begin{array}{ccc}
e & & 0 \\
& \ddots & \\
0 & & e
\end{array}\right)
$$

is an order unit in $M_{n}(V)$ for every $n$. We say that $e$ is Archimedean matrix order unit if it is a matrix order unit and satisfies the following: For any $v$ in $V$ if $\epsilon e+v$ is positive for every $\epsilon>0$ then $v$ is positive. A matrix ordered $*$-vector space $V$ (with cone structure $\left\{C_{n}\right\}$ ) and Archimedean matrix order unit $e$ is called an (abstract) operator system. We often drop the term "Archimedean matrix order" and simply use "unit" for $e$. We sometimes use the notation $\left(V,\left\{C_{n}\right\}, e\right)$ for an operator system however to avoid excessive syntax we simply prefer to use $\mathcal{S}$ (or $\mathcal{T}, \mathcal{R}$ ). The positive elements of $\mathcal{S}$, i.e. $C_{1}$, is denoted by $\mathcal{S}^{+}$and for the upper levels we use $M_{n}(\mathcal{S})^{+}$rather than $C_{n}$. Sometimes we use $e_{\mathcal{S}}$ for the unit. A subspace $V$ of $B(H)$ (or in general a unital $\mathrm{C}^{*}$-algebra $\mathcal{A}$ ) that contains the unit $I$ and is closed under $*$ (i.e. a unital selfadjoint subspace) is called a concrete operator system. Note that $V$ together with the induced matrix order structure, i.e. $C_{n}=M_{n}(V) \cap M_{n}(B(H))^{+}$for every $n$, and $I$ forms an (abstract) operator system. In the next paragraph we work on the morphisms of operator systems and see that abstract and concrete operator systems are "essentially" same.

Let $\mathcal{S}$ and $\mathcal{T}$ be two operator systems and $\varphi: \mathcal{S} \rightarrow \mathcal{T}$ be a linear map. We say that $\varphi$ is unital if $\varphi\left(e_{\mathcal{S}}\right)=e_{\mathcal{T}} \cdot \varphi$ is called positive if it maps positive elements of $\mathcal{S}$ to positive elements of $\mathcal{T}$, that is, $\varphi\left(\mathcal{S}^{+}\right) \subset \mathcal{T}^{+}$, and completely positive if its $n^{\text {th }}$-amplification $\varphi^{n}: M_{n}(\mathcal{S}) \rightarrow$ $M_{n}(\mathcal{T})$ given by $\left(s_{i j}\right) \mapsto\left(\varphi\left(s_{i j}\right)\right)$ is positive for every $n$, in other words, $\varphi^{n}\left(M_{n}(\mathcal{S})^{+}\right) \subset$ $M_{n}(\mathcal{T})^{+}$for all $n$. The term unital and completely positive will abbreviated as ucp. $\varphi$ will be called a complete order embedding if it is injective ucp map such that whenever $\left(\varphi\left(s_{i j}\right)\right)$ is positive in $M_{n}(\mathcal{T})$ then $\left(s_{i j}\right)$ is positive in $M_{n}(\mathcal{S})$. Two operator system $\mathcal{S}$ and $\mathcal{T}$ are called unitally completely order isomorphic if there is a bijective map $\varphi: \mathcal{S} \rightarrow \mathcal{T}$ that is unital and a complete order isomorphism. A subspace $\mathcal{S}_{0}$ of operator system $\mathcal{S}$ which is unital and selfadjoint is again an operator system together with the induced matrix order structure. In this case we say that $\mathcal{S}_{0}$ is an operator subsystem of $\mathcal{S}$. Note that the inclusion $\mathcal{S}_{0} \hookrightarrow \mathcal{S}$ is a unital complete order embedding. $\mathcal{O}$ stands for the category whose objects are the operator systems and morphisms are the ucp maps. We are now ready to state the celebrated theorem of Choi and Effros ([6]). 
Theorem 1.1. Up to a unital complete order isomorphism all the abstract and concrete operator systems coincide. That is, if $\mathcal{S}$ is an operator system then there is a Hilbert space $H$ and a unital $*$-linear map $\varphi: \mathcal{S} \rightarrow B(H)$ which is a complete order embedding.

Of course, in the above theorem $B(H)$ can be replaced with a unital $\mathrm{C}^{*}$-algebra. A subspace $X$ of a $\mathrm{C}^{*}$-algebra $\mathcal{A}$ together with the induced matrix norm structure is called a concrete operator space. We refer the reader to [33] for an introductory exposition of these objects along with their abstract characterization due to Ruan. If $\mathcal{S}$ is an operator system then a concrete representation of $\mathcal{S}$ into a $B(H)$ endows $\mathcal{S}$ with an operator space structure. It follows that this structure is independent of the particular representation and, moreover, it can be intrinsically given as

$$
\left\|\left(s_{i j}\right)\right\|_{n}=\inf \left\{\alpha>0:\left(\begin{array}{cc}
\alpha e_{n} & \left(s_{i j}\right) \\
\left(s_{j i}^{*}\right) & \alpha e_{n}
\end{array}\right) \text { is in } M_{2 n}(\mathcal{S})^{+}\right\} .
$$

This is known as the canonical operator space structure of $\mathcal{S}$. We also assume some familiarity with the injectivity in the category of operator systems. We refer to [33, Chp. 15] for an excellent survey, however for an immediate use in the sequel we remark that every injective operator system is completely order isomorphic to a $\mathrm{C}^{*}$-algebra [33, Thm 15.2]. We also need the fact that if $\mathcal{S}$ is an operator system then its injective envelope $I(\mathcal{S})$ is "rigid" in the sense that the only ucp map $\varphi: I(\mathcal{S}) \rightarrow I(\mathcal{S})$ with the property that $\varphi(s)=s$ for every $s$ in $\mathcal{S}$ is the identity [33, Thm 15.7].

\subsection{Some Special C*-covers.}

A $\mathrm{C}^{*}$-cover $(\mathcal{A}, i)$ of an operator system $\mathcal{S}$ is a $\mathrm{C}^{*}$-algebra $\mathcal{A}$ with a unital complete order embedding $i: \mathcal{S} \hookrightarrow \mathcal{A}$ such that $i(\mathcal{S})$ generates $\mathcal{A}$ as a $\mathrm{C}^{*}$-algebra, that is, $\mathcal{A}$ is the smallest $\mathrm{C}^{*}$ algebra containing $i(\mathcal{S})$. We occasionally identify $\mathcal{S}$ with $i(\mathcal{S})$ and consider $\mathcal{S}$ as an operator subsystem of $\mathcal{A}$. Every operator system $\mathcal{S}$ attains two special $\mathrm{C}^{*}$-covers namely the universal and the enveloping $\mathrm{C}^{*}$-algebras denoted by $C_{u}^{*}(\mathcal{S})$ and $C_{e}^{*}(\mathcal{S})$, respectively. The universal $\mathrm{C}^{*}$ algebra satisfies the following universal "maximality" property: Every ucp map $\varphi: \mathcal{S} \rightarrow \mathcal{A}$, where $\mathcal{A}$ is a $\mathrm{C}^{*}$-algebra extends uniquely to a unital $*$-homomorphism $\pi: C_{u}^{*}(\mathcal{S}) \rightarrow \mathcal{A}$. If $\varphi: \mathcal{S} \rightarrow \mathcal{T}$ is a ucp map then the unital $*$-homomorphism $\pi: C_{u}^{*}(\mathcal{S}) \rightarrow C_{u}^{*}(\mathcal{T})$ associated with $\varphi$, of course, constructed by enlarging the range space by $C_{u}^{*}(\mathcal{T})$ first. We also remark that if $\mathcal{S} \subset \mathcal{T}$ then $C_{u}^{*}(\mathcal{S}) \subset C_{u}^{*}(\mathcal{T})$, in other words, the $\mathrm{C}^{*}$-algebra generated by $\mathcal{S}$ in $C_{u}^{*}(\mathcal{T})$ coincides with the universal $\mathrm{C}^{*}$-algebra of $\mathcal{S}$. This special $\mathrm{C}^{*}$-cover is used extensively in [22, 21] and [27]. As it connects operator systems to $\mathrm{C}^{*}$-algebras it has fundamental role in the tensor theory of operator systems and, in particular, in the present paper.

The enveloping $\mathrm{C}^{*}$-algebra $C_{e}^{*}(\mathcal{S})$ of $\mathcal{S}$ is defined as the $\mathrm{C}^{*}$-algebra generated by $\mathcal{S}$ in its injective envelope $I(\mathcal{S})$. It has the following universal "minimality" property: For any $\mathrm{C}^{*}$-cover $i: \mathcal{S} \hookrightarrow \mathcal{A}$ there is a unique unital $*$-homomorphism $\pi: \mathcal{A} \rightarrow C_{e}^{*}(\mathcal{S})$ such that $\pi(i(s))=s$ for every $s$ in $\mathcal{S}$ (we assume $\mathcal{S} \subset C_{e}^{*}(\mathcal{S})$ ). The enveloping $\mathrm{C}^{*}$-algebra of an operator system is rigid in the sense that if $\varphi: C_{e}^{*}(\mathcal{S}) \rightarrow \mathcal{T}$ is a ucp map such that $\left.\varphi\right|_{\mathcal{S}}$ is a complete order embedding then $\varphi$ is a complete order embedding. We refer to [15] for the proof of these results and further properties of enveloping $\mathrm{C}^{*}$-algebras.

\subsection{Duality.}

Duality, especially on the finite dimensional operator systems, is a strong tool in the study of the stability of various nuclearity properties and in this subsection we review basic properties on this topic. If $\mathcal{S}$ is an operator system then the Banach dual $\mathcal{S}^{d}$ has a natural matrix 
ordered $*$-vector space structure. For $f$ in $\mathcal{S}^{d}$, the involution is given by $f^{*}(s)=\overline{f\left(s^{*}\right)}$. The matricial order structure is described as follows:

$$
\left(f_{i j}\right) \in M_{n}\left(\mathcal{S}^{d}\right) \text { is positive if the map } \mathcal{S} \ni s \mapsto\left(f_{i j}(s)\right) \in M_{n} \text { is completely positive. }
$$

Throughout the paper $\mathcal{S}^{d}$ will always represent this matrix ordered vector space. The bidual Banach space $\mathcal{S}^{d d}$ has also a natural matricial order structure arising from the fact that it is the dual of $\mathcal{S}^{d}$. The following is perhaps well known, see [22], e.g.:

Theorem 1.2. $\mathcal{S}^{d d}$ is an operator system with unit $\hat{e}$, the canonical image of $e$ in $\mathcal{S}^{d d}$. Moreover, the canonical embedding of $\mathcal{S}$ into $\mathcal{S}^{\text {dd }}$ is a complete order embedding.

A state $f$ on $\mathcal{S}$ is said to be faithful if $s \geq 0$ and $f(s)=0$ implies that $s=0$, in other words, $f$ maps non-zero positive elements to positive scalars. When $\mathcal{S}$ is a finite dimensional operator system then it possesses a faithful state which is an Archimedean matrix order unit for the dual structure [6, Sec. 4]:

Theorem 1.3 (Choi-Effros). Suppose $\mathcal{S}$ is a finite dimensional operator system. Then there are faithful states on $\mathcal{S}$ and each faithful state is an Archimedean order unit for the matrix ordered space $\mathcal{S}^{d}$.

Consequently, the dual of a finite dimensional operator system is again an operator system when we fix a faithful state. Also note that when we pass to the second dual, $\hat{e} \in \mathcal{S}^{d d}$ is a faithful state on $\mathcal{S}^{d}$. The following will be useful in later sections:

Lemma 1.4. Let $\mathcal{S}$ and $\mathcal{T}$ be two operator systems and $\varphi: \mathcal{S} \rightarrow \mathcal{T}$ be a linear map. Then $\varphi$ is $k$-positive if and only if $\varphi^{d}: \mathcal{T}^{d} \rightarrow \mathcal{S}^{d}$ is $k$-positive.

Proof. First suppose that $\varphi$ is $k$-positive. Let $\left(g_{i j}\right)$ be in $M_{k}\left(\mathcal{T}^{d}\right)^{+}$. We need to show that $\left(\varphi^{d}\left(g_{i j}\right)\right)$ is in $M_{k}\left(\mathcal{S}^{d}\right)^{+}$, that is, the map

$$
\mathcal{S} \ni s \mapsto\left(\varphi^{d}\left(g_{i j}(s)\right)\right)=\left(g_{i j}(\varphi(s))\right) \in M_{k}
$$

is completely positive. By using a result of Choi, see [33, Thm. 6.1] e.g., it is enough to show that this map is $k$-positive. So let $\left(s_{l m}\right)$ be positive in $M_{k}(\mathcal{S})$. Since $\varphi$ is $k$-positive we have that $\left(\varphi\left(s_{l m}\right)\right)$ is positive in $M_{n}(\mathcal{T})$. Now using the definition of positivity of $\left(g_{i j}\right)$ we have that $\left(g_{i j}\left(\varphi\left(s_{l m}\right)\right)\right)$ is positive in $M_{k}\left(M_{k}\right)$. Conversely, suppose that $\varphi^{d}$ is $k$-positive. By using the above argument, we have that $\varphi^{d d}: \mathcal{S}^{d d} \rightarrow \mathcal{T}^{d d}$ is $k$-positive. Since $\mathcal{S} \subset \mathcal{S}^{d d}$ and $\mathcal{T} \subset \mathcal{T}^{d d}$ completely order isomorphically we have that $\varphi=\left.\varphi^{d d}\right|_{\mathcal{S}}$ is $k$-positive.

\section{Operator System Quotients}

In this section we recall some basic results about operator system quotients introduced in [21, Sec. 3, 4]. This quotient theory is also studied and used extensively in [10] and some of them are included in the sequel. We exhibit some relations between the quotient theory and duality for finite dimensional operator systems. We establish some universal objects, namely the coproducts of operator systems, by using the quotient theory in a later section.

A subspace $J$ of an operator system $\mathcal{S}$ is called a kernel if it is the kernel of some ucp map defined from $\mathcal{S}$ into an operator system $\mathcal{T}$. Note that a kernel $J$ has to be a $*$-closed, non-unital subspace of $\mathcal{S}$, however, these properties, in general, do not characterize a kernel. The following is Proposition 3.2 of [21].

Proposition 2.1. Let $J$ be a subspace of $\mathcal{S}$. Then the following are equivalent: 
(1) $J$ is a kernel,

(2) $J$ is the kernel of a cp map defined from $\mathcal{S}$ into an operator system $\mathcal{T}$,

(3) $J$ is the kernel of a positive map defined from $\mathcal{S}$ into an operator system $\mathcal{T}$,

(4) there is a collection of states $f_{\alpha}$ such that $J=\cap_{\alpha} k e r\left(f_{\alpha}\right)$.

The algebraic quotient $\mathcal{S} / J$ has a natural involution given by $(s+J)^{*}=s^{*}+J$. To define the matricial order structure we first consider the following cones:

$$
D_{n}=\left\{\left(s_{i j}+J\right)_{i, j=1}^{n}: \quad\left(s_{i j}\right) \in M_{n}(\mathcal{S})^{+}\right\} .
$$

It is elementary to show that $\left\{D_{n}\right\}_{n=1}^{\infty}$ forms a strict, compatible order structure. Moreover, $e+J$ is a matrix order unit. However, it fails to be Archimedean, that is, if $(s+J)+\epsilon(e+J)$ is in $D_{1}$ for every $\epsilon>0$, then $s+J$ may not be in $D_{1}$. To solve this problem we use the Archimedeanization process introduced in [37. More precisely, we enlarge the cones in such a way that they still form a strict compatible matricial order structure and $e+J$ is an Archimedean matrix order unit. Consider

$$
C_{n}=\left\{\left(s_{i j}+J\right)_{i, j=1}^{n}:\left(s_{i j}\right)+\epsilon(e+J)_{n} \in D_{n} \text { for every } \epsilon>0\right\} .
$$

The *-vector space $\mathcal{S} / J$ together with the matricial order structure $\left\{C_{n}\right\}_{n=1}^{\infty}$ and unit $e+J$ form an operator system. We refer to [21, Sec. 3] for the proof of this result. The operator system $\mathcal{S} / J$ is called the quotient operator system. A kernel $J$ is called proximinal if $D_{1}=C_{1}$ and completely proximinal if $D_{n}=C_{n}$ for every $n$. We remark that the proximinality in this context is different than the norm-proximinality in the Banach or operator space quotients.

One of the fundamental properties of an operator system quotient $\mathcal{S} / J$ is its relation with morphisms. If $\varphi: \mathcal{S} \rightarrow \mathcal{T}$ is a ucp map with $J \subseteq \operatorname{ker}(\varphi)$ then the associated map $\bar{\varphi}: \mathcal{S} / J \rightarrow T$ is again a ucp map. Conversely, if $\psi: \mathcal{S} / J \rightarrow \mathcal{T}$ is a ucp map then there exists a unique ucp map $\phi: \mathcal{S} \rightarrow \mathcal{T}$ with, necessarily, $J \subseteq \operatorname{ker}(\phi)$ such that $\phi=q \circ \psi$ where $q$ is the quotient map from $\mathcal{S}$ onto $\mathcal{S} / J$. We also remark that if one considers completely positive maps and drop the condition on the unitality then both of these universal properties still hold.

Remark: If one starts with a $*$-closed, non-unital subspace $J$ of an operator system $\mathcal{S}$ then, on the algebraic quotient $\mathcal{S} / J$ the involution is still well-defined. We can still define $D_{n}$ in similar fashion and it is elementary to show that $\left\{D_{n}\right\}$ is a compatible matricial cone structure. It is possible that $\left\{D_{n}\right\}$ is strict as well. However, in order to obtain the Archimedeanization property of $e+J$ we again need to enlarge the cones and define $\left\{C_{n}\right\}$ in a similar way. Now it can be shown that $C_{1}$ is strict, that is, $C_{1} \cap\left(-C_{1}\right)=\{0\}$, if and only if $J$ is a kernel. Consequently starting with a kernel is essential in the operator system quotient. (See [21, Sec. 3] for an extended discussion on this topic).

Remark 2.2. Let $\mathcal{A}$ be a unital $\mathrm{C}^{*}$-algebra and $I$ be an ideal in $\mathcal{A}$. (It is easy to see that $I$ is a kernel, in fact it is the kernel of the quotient map $\mathcal{A} \rightarrow \mathcal{A} / I$ ). Then the $\mathrm{C}^{*}$-algebraic quotient of $\mathcal{A}$ by $I$ is unitally completely order isomorphic to the operator system quotient $\mathcal{A} / I$. Moreover, $I$ is proximinal.

Proximinality is a useful tool and we want to consider some special cases in which the kernels are automatically proximinal. The first part of the following is essentially [21, Lemma 4.3.].

Lemma 2.3. Let y be a selfadjoint element of an operator system $\mathcal{S}$ which is neither positive nor negative. Then $\operatorname{span}\{y\}$ is a kernel in $\mathcal{S}$. Moreover, span $\{y\}$ is proximinal. 
Proof. The first part of the proof can be found in [21, Lemma 4.3]. To prove the second part we first consider the case where $y$ is such an element in a unital $\mathrm{C}^{*}$-algebra $\mathcal{A}$. Let $J=\operatorname{span}\{y\}$ and let $x+J \geq 0$ in $\mathcal{A} / J$. Clearly we may assume that $x=x^{*}$. We have that for each $\epsilon>0$ there is an element in $J$, say $\alpha_{\epsilon} y$ such that $x+\alpha_{\epsilon} y+\epsilon e$ is positive in $\mathcal{A}$. Note that $\alpha_{\epsilon}$ must be a real number. Let $X_{\epsilon}=\left\{\alpha: x+\alpha y+\epsilon e \in \mathcal{A}^{+}\right\}$then $X_{\epsilon}$ is a non-empty subset of $\mathbb{R}$ such that for any $0<\epsilon_{1} \leq \epsilon_{2}$ we have $X_{\epsilon_{1}} \subseteq X_{\epsilon_{2}}$. Moreover since $\mathcal{A}^{+}$is closed in $\mathcal{A}$, each of $X_{\epsilon}$ is closed. We will show that $X_{1}$ is bounded. Let $y=y_{1}-y_{2}$ be the Jordan decomposition of $y$, that is, $y_{1}$ and $y_{2}$ are positives such that $y_{1} y_{2}=0$. Let $\alpha$ be in $X_{1}$. Now multiplying both side of $x+\alpha y_{1}-\alpha y_{2}+e \geq 0$ by $y_{2}$ from right and left we get $y_{2} x y_{2}+y_{2}^{2} \geq \alpha y_{2}^{3}$. Since $y_{2}$ is non-zero this inequality puts an upper bound on $\alpha$. Similarly multiplying both side by $y_{1}$ we obtain a lower bound for $\alpha$. Consequently $\left\{X_{\epsilon}\right\}_{0<\epsilon \leq 1}$ is a decreasing net of compact sets in $\mathbb{R}$ and hence have a non-empty intersection. Let $\alpha_{0}$ be an element belongs to the intersection. Since $x+\alpha_{0} y+\epsilon \geq 0$ for every $\epsilon>0$ we have that $x+\alpha_{0} y \geq 0$. This proves the particular case we assumed. Now suppose $y$ is such an element in $\mathcal{S}$. Let $\mathcal{A}$ be a $\mathrm{C}^{*}$-algebra containing $\mathcal{S}$ as an operator subsystem. We have that $J=\operatorname{span}\{y\}$ is a proximinal kernel in $\mathcal{A}$. Let $q$ be the quotient map from $\mathcal{A}$ onto $\mathcal{A} / J$ and let $q_{0}$ be the restriction of $q$ on $\mathcal{S}$. Clearly $q_{0}$ is ucp with kernel $J$. So $\overline{q_{0}}: \mathcal{S} / J \rightarrow \mathcal{A} / J$ is ucp. Now let $s+J$ be positive in $\mathcal{S} / J$. So it is positive in $\mathcal{A} / J$. By the above part there is an element $a$ in $\mathcal{A}^{+}$such that $a+J=s+J$. Since $J$ is contained $\mathcal{S}$ clearly $a$ must be an element of $\mathcal{S}$. So the proof is done.

A finite dimensional $*$-closed subspace $J$ of an operator system $\mathcal{S}$ which contains no positive other than 0 is called a null subspace. Supposing $y$ is a self-adjoint element of $\mathcal{S}$ which is neither positive nor negative then $\operatorname{span}\{y\}$ is a one dimensional null subspace, e.g. Another important example of null subspaces are kernels of faithful states on finite dimensional operator systems.

Proposition 2.4. Suppose $J$ is a null subspace of $\mathcal{S}$. Then $J$ is a completely proximinal kernel. If $\mathcal{S}$ is finite dimensional, say $\operatorname{dim}(\mathcal{S})=n$, then $J$ is contained in an $n-1$ dimensional null subspace.

Proof. We first show that $J$ is a proximinal kernel. We will argue by induction on the dimension of $J$. When $J$ is one dimensional Lemma 2.3 does the job. Suppose every $k$ dimensional null subspace of the operator system $\mathcal{S}$ is a proximinal kernel and let $J$ be an $k+1$ dimensional null subspace. It is elementary to see that $J=\operatorname{span}\left\{y_{1}, \ldots, y_{k}, y_{k+1}\right\}$ where each of $y_{i}$ is selfadjoint. Let $J_{0}=\operatorname{span}\left\{y_{1}, \ldots, y_{k}\right\}$ which is a null subspace and consequently a proximinal kernel by the induction assumption. We claim that $y_{k+1}+J_{0}$ is a selfadjoint element in $\mathcal{S} / J_{0}$ which is neither positive nor negative. Clearly it is selfadjoint. Suppose it is positive, so there is a positive element $x$ in $\mathcal{S}$ such that $x+J_{0}=y_{k+1}+J_{0}$. This clearly forces $x$ to be in $J$ so it is necessarily 0 and thus $y_{k+1}$ is in $J_{0}$ which is a contradiction. Similarly $y_{k+1}$ cannot be negative. Again by using Lemma $2.3 \operatorname{span}\left\{y_{k+1}+J_{0}\right\}$ is a proximinal kernel in $\mathcal{S} / J_{0}$. Now consider the sequence of the quotients maps

$$
\mathcal{S} \stackrel{q_{0}}{\longrightarrow} \mathcal{S} / J_{0} \stackrel{q_{1}}{\longrightarrow}\left(\mathcal{S} / J_{0}\right) / \operatorname{span}\left\{y_{k+1}+J_{0}\right\}
$$

Clearly the kernel of $q_{1} \circ q_{0}$ is $J$ and since the first and the second quotients are proximinal it is easy to show that that $J$ is proximinal. To see that $J$ is a completely proximinality we can simply consider the identification

$$
M_{n}(\mathcal{S} / J)=M_{n}(\mathcal{S}) / M_{n}(J) .
$$

Note that $M_{n}(J)$ is still a null subspace on $M_{n}(\mathcal{S})$. 
Now we will show that if $\operatorname{dim}(\mathcal{S})=n$ then $J$ is contained in an $n-1$ dimensional null subspace. Let $w$ be a faithful state on $\mathcal{S} / J$. Clearly kernel of $w$ is a null subspace and so proximinal by the above part. Now;

$$
\mathcal{S} \stackrel{q}{\longrightarrow} \mathcal{S} / J \stackrel{w}{\longrightarrow} \mathbb{C}
$$

is a sequence of ucp maps with $n-1$ dimensional kernel in $\mathcal{S}$ which contains $J$. It is null subspace since a non-zero positive will map a non-zero positive by $q$ first and a non-zero positive real number by $w$.

As we pointed out earlier, the kernel of a faithful state on a finite dimensional operator system is a null subspace. This led us to construct a very special basis for the operator system as well as its dual.

Lemma 2.5. Suppose $\mathcal{S}$ is an $n$ dimensional operator system and $\delta$ a faithful state on $\mathcal{S}$. Then the kernel of $\delta$, which is an $n-1$ dimensional null subspace, can be written as a linear combination of self-adjoint elements $\left\{s_{2}, \ldots, s_{n}\right\}$. Consequently we have

$$
\mathcal{S}=\operatorname{span}\left\{e=s_{1}, s_{2}, \ldots, s_{n}\right\} .
$$

Moreover if $\mathcal{S}^{d}=\operatorname{span}\left\{\delta=\delta_{1}, \delta_{2}, \ldots ., \delta_{n}\right\}$ written in the dual basis form (i.e. $\delta_{i}\left(s_{j}\right)=\delta_{i j}$ ) then $\delta_{2}, \ldots, \delta_{n}$ are self-adjoint elements of the dual operator system such that their span is a null subspace.

Proof. It is elementary to see that the kernel of $\delta$ can be written as a linear combination of selfadjoints. In fact we can start with a selfadjoint element $s_{2}$. If $s$ is an element in the kernel which is not in the span of $s_{2}$ then one of $s+s^{*}$ or $\left(s-s^{*}\right) i$ does not belong to span of $s_{2}$. So this way we obtain $s_{3}$. We can apply this procedure successively and form such a basis. Clearly if we set $s_{1}=e$ then we obtain a basis for $\mathcal{S}$. To see that $\delta_{i}$ is self-adjoint consider an element $\Sigma \alpha_{j} s_{j}$. Then

$$
\delta_{i}^{*}\left(\Sigma \alpha_{j} s_{j}\right)=\overline{\delta_{i}\left(\Sigma\left(\alpha_{j} s_{j}\right)^{*}\right)}=\overline{\delta_{i}\left(\sum \overline{\alpha_{j}} s_{j}\right)}=\alpha_{i}
$$

coincides with $\delta_{i}\left(\Sigma \alpha_{j} s_{j}\right)$. Finally since $\hat{e}$, the canonical image of $e$ in the bidual operator system, is a faithful state on the dual operator system $\mathcal{S}^{d}$ its kernel, namely the linear span of $\left\{\delta_{2}, \ldots, \delta_{n}\right\}$, is a null subspace. This finishes the proof.

Let $J_{n}$ be the subspace of $M_{n}$ containing all diagonal matrices with 0 trace. Then $J_{n}$ is an $n-1$ dimensional null subspace of $M_{n}$ and consequently a kernel. Note that it is contained in the subspace which includes all the matrices with 0 trace, an $n^{2}-1$ dimensional null subspace of $M_{n}$. In [10] it has been explicitly shown that $J_{n}$ is a kernel. We will turn back to this in later sections. Another interesting example is the following: Consider $J=\operatorname{span}\left\{g_{1}, \ldots, g_{n}, g_{1}^{*}, \ldots, g_{n}^{*}\right\} \subset C^{*}\left(\mathbb{F}_{n}\right)$. Then $J$ is a null subspace and hence a kernel in $C^{*}\left(\mathbb{F}_{n}\right)$.

A surjective completely positive map $\varphi: \mathcal{S} \rightarrow \mathcal{T}$ is called a quotient map if the induced map $\bar{\varphi}: \mathcal{S} / \operatorname{ker}(\varphi) \rightarrow \mathcal{T}$, which is bijective and completely positive, is a complete order isomorphism. Note that if $\varphi$ is unital the induced map is also unital. We also remark that compositions of quotient maps are again quotient maps. We frequently use the following property of a quotient map: If $\left(t_{i j}\right)$ is positive in $M_{k}(\mathcal{T})$ then for every $\epsilon>0$ there is a positive element $\left(s_{i j}^{\epsilon}\right)$ in $M_{k}(\mathcal{S})$ such that $\left(\varphi\left(s_{i j}^{\epsilon}\right)\right)=\left(t_{i j}\right)+\epsilon e_{n}$.

Proposition 2.6. Let $\varphi: \mathcal{S} \rightarrow \mathcal{T}$ be a quotient map. Then the dual map $\varphi^{d}: \mathcal{T}^{d} \rightarrow \mathcal{S}^{d}$ is a complete order embedding. 
Proof. We already have that the dual map is completely positive. Suppose $\left(g_{i j}\right)$ in $M_{n}\left(\mathcal{T}^{d}\right)$ such that $\left(\varphi^{d}\left(g_{i j}\right)\right)$ is positive in $M_{n}\left(\mathcal{S}^{d}\right)$. We will show that $\left(g_{i j}\right)$ is positive, that is, if $\left(t_{l m}\right)$ is positive in $M_{k}(\mathcal{T})$ then $\left(g_{i j}\left(t_{l m}\right)\right)$ is positive (in $\left.M_{k} \otimes M_{n}\right)$. Fix $\epsilon>0$ and let $\left(t_{l m}^{\epsilon}\right)=\left(t_{l m}\right)+\epsilon e_{k}$. We know that there is positive element $\left(s_{l m}^{\epsilon}\right)$ in $M_{k}(\mathcal{S})$ such that $\left(\varphi\left(s_{l m}^{\epsilon}\right)\right)=\left(t_{l m}^{\epsilon}\right)$. Note that $\left(g_{i j}\left(t_{l m}^{\epsilon}\right)\right)_{i, j, l, m}=\left(\varphi^{d}\left(g_{i j}\right)\left(s_{l m}^{\epsilon}\right)\right)$. Now using the fact that $\left(\varphi^{d}\left(g_{i j}\right)\right)$ is positive we get $\left(g_{i j}\left(t_{l m}^{\epsilon}\right)\right)_{i, j, l, m}$ is positive. Since $\epsilon$ is arbitrary and $\left(t_{l m}^{\epsilon}\right) \rightarrow\left(t_{l m}\right)$ as $\epsilon \rightarrow 0$ we have that $\left(g_{i j}\left(t_{l m}\right)\right)$ is positive. So the proof is done.

Proposition 2.7. Let $J$ be a null subspace of a finite dimensional operator system $\mathcal{S}$. Then $(\mathcal{S} / J)^{d}$ is an operator subsystem of $\mathcal{S}^{d}$ with a proper selection of faithful states. (More precisely if $\delta$ is a faithful state on $\mathcal{S}$ with $J \subset \operatorname{ker}(\delta)$ then the induced state $\bar{\delta}$ on $\mathcal{S} / J$ is faithful and satisfies $q^{d}(\bar{\delta})=\delta$ where $q$ is the quotient map from $\mathcal{S}$ onto $\left.\mathcal{S} / J\right)$.

Proof. Proposition 2.6 ensures that $q^{d}:(\mathcal{S} / J)^{d} \rightarrow \mathcal{S}^{d}$ is a complete order embedding. So we deal with the proper selection of the faithful states. In fact let $\delta_{0}$ be a faithful state on $\mathcal{S} / J$. Then we claim that $\delta_{0} \circ q$ is a faithful state on $\mathcal{S}$. Clearly it is a state and if $s$ is non-zero positive then $\varphi(s)$ is non-zero positive in $\mathcal{S} / J$ and $\delta_{0}(q(s))$ is a positive number. Finally declaring $\delta_{0} \circ q$ as the unit of $\mathcal{S}^{d}$, we obtain that $q^{d}$ is unital as $q^{d}\left(\delta_{0}\right)=\delta_{0} \circ q$.

We remark that in order to obtain "unitality" in the above proposition starting with a null subspace is important. In fact if $J$ is a kernel and $\delta_{1}$ and $\delta_{2}$ are faithful states on $\mathcal{S} / J$ and $\mathcal{S}$, respectively, then $q^{d}\left(\delta_{1}\right)=\delta_{2}$ requires that $J$ is in the kernel of $\delta_{2}$ and consequently it has to be a null subspace.

The converse of the above result is also true which is referred as the First Isomorphism Theorem in [10]. For completeness of the paper we include the proof.

Theorem 2.8 (Farenick, Paulsen). Let $\mathcal{S}$ be a finite dimensional operator system and $\mathcal{S}_{0}$ be an operator subsystem of $\mathcal{S}$. Then the adjoint $i^{d}: \mathcal{S}^{d} \rightarrow \mathcal{S}_{0}^{d}$ of the inclusion $\mathcal{S}_{0} \hookrightarrow \mathcal{S}$ is a quotient map. By proper selection of faithful states we may also assume that it is unital.

Proof. Since the inclusion is a cp map its adjoint is again a cp map. It is also elementary to see that $i^{d}$ is surjective. Thus, we will only prove that if $\left(i^{d}\left(f_{i j}\right)\right)$ is positive in $M_{n}\left(\mathcal{S}_{0}^{d}\right)$ then there is positive $\left(g_{i j}\right)$ in $M_{n}(\mathcal{S})$ such that $i^{d}\left(f_{i j}\right)=i^{d}\left(g_{i j}\right)$ for every $i, j$. Now, $\left(i^{d}\left(f_{i j}\right)\right)$ is positive in $M_{n}\left(\mathcal{S}_{0}^{d}\right)$ means that the linear map

$$
\mathcal{S}_{0} \ni s \mapsto\left(i^{d}\left(f_{i j}\right)(s)\right)=\left(f_{i j}(s)\right) \in M_{n}
$$

is a cp map. By Arveson's extension theorem ([1]), this map has a cp extension from $\mathcal{S}$ into $M_{n}$, which we identify with $\left(g_{i j}\right)$. Now, clearly $\left(g_{i j}\right)$ is positive in $M_{n}\left(\mathcal{S}^{d}\right)$ and $i^{d}\left(f_{i j}\right)=i^{d}\left(g_{i j}\right)$ for every $i, j$. We will continue with the unitality problem. In fact it is elementary to show that if $f$ is a faithful state on $\mathcal{S}$ then $f$ still has the same property when it is restricted to $\mathcal{S}_{0}$. Thus $i^{d}(f)$ is again a faithful state.

Remark 2.9. In the above theorem we see that adjoint of the inclusion map is a unital quotient map. The kernel of this map is a null subspace. In fact if $f$ is positive in $\mathcal{S}^{d}$ and $i^{d}(f)=0$ together imply that $f$ is a positive linear functional on $\mathcal{S}$ such that $\left.f\right|_{\mathcal{S}_{0}}$ is 0 . Since, we have that $\|f\|=\|f(e)\|$, necessarily $f=0$.

\section{Tensor Products of Operator Systems}

In this section we recall the axiomatic definition of tensor products in the category of operator systems and review properties of several tensor products established in 22]. Suppose 
$\mathcal{S}$ and $\mathcal{T}$ are two operator systems. A matricial cone structure $\tau=\left\{C_{n}\right\}$ on $\mathcal{S} \otimes \mathcal{T}$ where $C_{n} \subset M_{n}(\mathcal{S} \otimes \mathcal{T})_{h}$, is said to be an operator system structure if

(1) $\left(\mathcal{S} \otimes \mathcal{T},\left\{C_{n}\right\}, e_{\mathcal{S}} \otimes e_{\mathcal{T}}\right)$ is an operator system,

(2) for any $\left(s_{i j}\right) \in M_{n}(\mathcal{S})^{+}$and $\left(t_{r s}\right) \in M_{k}(\mathcal{T})^{+},\left(s_{i j} \otimes t_{r s}\right)$ is in $C_{n k}$ for all $n, k$,

(3) if $\phi: \mathcal{S} \rightarrow M_{n}$ and $\psi: \mathcal{T} \rightarrow M_{k}$ are ucp maps then $\phi \otimes \psi: \mathcal{S} \otimes \mathcal{T} \rightarrow M_{n k}$ is a ucp map for every $n$ and $k$.

The resulting operator system is denoted by $\mathcal{S} \otimes_{\tau} \mathcal{T}$. A mapping $\tau: \mathcal{O} \times \mathcal{O} \rightarrow \mathcal{O}$ is said to be an operator system tensor product (or simply a tensor product) provided $\tau$ maps each pair $(\mathcal{S}, \mathcal{T}$ ) to an operator system structure on $\mathcal{S} \otimes \mathcal{T}$, denoted by $\mathcal{S} \otimes_{\tau} \mathcal{T}$. A tensor product $\tau$ is said to be functorial if for every operator systems $\mathcal{S}_{1}, \mathcal{S}_{2}, \mathcal{T}_{1}$ and $\mathcal{T}_{2}$ and every ucp maps $\phi: \mathcal{S}_{1} \rightarrow \mathcal{S}_{2}$ and $\psi: \mathcal{T}_{1} \rightarrow \mathcal{T}_{2}$ the associated map $\phi \otimes \psi: \mathcal{S}_{1} \otimes_{\tau} \mathcal{T}_{1} \rightarrow \mathcal{S}_{2} \otimes_{\tau} \mathcal{T}_{2}$ is ucp. A tensor product $\tau$ is called symmetric if $\mathcal{S} \otimes_{\tau} \mathcal{T}=\mathcal{T} \otimes_{\tau} \mathcal{S}$ and associative if $\left(\mathcal{S} \otimes_{\tau} \mathcal{T}\right) \otimes_{\tau} \mathcal{R}=\mathcal{S} \otimes_{\tau}\left(\mathcal{T} \otimes_{\tau} \mathcal{R}\right)$ for every $\mathcal{S}, \mathcal{T}$ and $\mathcal{R}$.

There is a natural partial order on the operator system tensor products: If $\tau_{1}$ and $\tau_{2}$ are two tensor products then we say that $\tau_{1} \leq \tau_{2}$ if for every operator systems $\mathcal{S}$ and $\mathcal{T}$ the identity $i d: \mathcal{S} \otimes_{\tau_{2}} \mathcal{T} \rightarrow \mathcal{S} \otimes_{\tau_{1}} \mathcal{T}$ is completely positive. In other words $\tau_{1}$ is smaller with respect to $\tau_{2}$ if the cones it generates are larger. (Recall that larger matricial cones generate smaller canonical operator space structure.) The partial order on operator system tensor products forms a lattice as pointed out in [22, Sec. 7] and raises fundamental nuclearity properties as we shall discuss in the next section.

In the remaining of this section we discuss several important tensor products, namely the minimal (min), maximal (max), maximal commuting (c), enveloping left (el) and enveloping right (er) tensor products. With respect to the partial order relation given in the previous paragraph we have the following schema [22] :

$$
\min \leq e l, \text { er } \leq c \leq \max .
$$

\subsection{Minimal Tensor Product.}

Let $\mathcal{S}$ and $\mathcal{T}$ be two operator systems. We define the matricial cone structure on the tensor product $\mathcal{S} \otimes \mathcal{T}$ as follows:

$$
\begin{aligned}
C_{n}^{\text {min }}(\mathcal{S}, \mathcal{T})=\left\{\left(u_{i j}\right)\right. & \in M_{n}(\mathcal{S} \otimes \mathcal{T}):\left((\phi \otimes \psi)\left(u_{i j}\right)\right)_{i j} \in M_{n k m}^{+} \\
& \text {for every ucp maps } \left.\phi: \mathcal{S} \rightarrow M_{k} \text { and } \psi: \mathcal{T} \rightarrow M_{m} \text { for all } k, m .\right\} .
\end{aligned}
$$

The resulting cone structure $\left\{C_{n}^{\text {min }}\right\}$ satisfies the axioms (1), (2) and (3) and the resulting operator system is denoted by $\mathcal{S} \otimes_{\min } \mathcal{T}$. If $\tau$ is another operator system structure on $\mathcal{S} \otimes \mathcal{T}$ then we have that $\min \leq \tau$. In other words $\left\{C_{n}^{\text {min }}\right\}$ forms the largest cone structure. The minimal tensor product, of course when considered as a map min $: \mathcal{O} \times \mathcal{O} \rightarrow \mathcal{O}$, is symmetric and associative. It is functorial and injective in the sense that if $\mathcal{S}_{1} \subset \mathcal{S}_{2}$ and $\mathcal{T}_{1} \subset \mathcal{T}_{2}$ then $\mathcal{S}_{1} \otimes_{\min } \mathcal{T}_{1} \subset \mathcal{S}_{2} \otimes_{\min } \mathcal{T}_{2}$ completely order isomorphically. It coincides with the the $\mathrm{C}^{*}$ algebraic minimal tensor products when restricted to $\mathrm{C}^{*}$-algebras (except for completion). It is also spatial in the sense that if $\mathcal{S} \subset B(H)$ and $\mathcal{T} \subset B(K)$ then the concrete operator system structure on $\mathcal{S} \otimes \mathcal{T}$ arising from the inclusion $B(H \otimes K)$ coincides with their minimal tensor product. All of these result can be directly found in [22, Sec. 4].

\subsection{Maximal Tensor Product.}

The construction of the maximal tensor product of two operator systems $\mathcal{S}$ and $\mathcal{T}$ involves two steps. We first define

$$
D_{n}^{\max }(\mathcal{S}, \mathcal{T})=\left\{A^{*}(P \otimes Q) A: P \in M_{k}(\mathcal{S})^{+}, Q \in M_{m}(\mathcal{T})^{+}, A \in M_{k m, n}, k, m \in \mathbb{N}\right\}
$$


The matricial order structure $\left\{D_{n}^{\max }\right\}$ is strict and compatible (for the definitions see [33, Chp. 13] e.g.), moreover, $e_{\mathcal{S}} \otimes e_{\mathcal{T}}$ is a matrix order unit. However it fails to be an Archimedean order unit. So the construction requires another step, namely the completion of the cones which is known as the Archimedeanization process (see [37] e.g) as follows:

$$
C_{n}^{\max }(\mathcal{S}, \mathcal{T})=\left\{P \in M_{n}(\mathcal{S} \otimes \mathcal{T}): r\left(e_{1} \otimes e_{2}\right)_{n}+P \in D_{n}^{\max }(\mathcal{S}, \mathcal{T}) \forall r>0\right\} .
$$

Now the matrix order structure $\left\{C_{n}^{\max }\right\}$ satisfies all the axioms and the resulting operator system is denoted by $\mathcal{S} \otimes_{\max } \mathcal{T}$. If $\tau$ is another operator system structure on $\mathcal{S} \otimes \mathcal{T}$ then we have that $\tau \leq \max$, that is, $\left\{C_{n}^{\max }\right\}$ is the smallest cone structure. max, as min, has all properties symmetry, associativity and functoriality. It coincides with the $\mathrm{C}^{*}$-algebraic maximal tensor product when restricted to unital $\mathrm{C}^{*}$-algebras (again, except for completion). As it is well known from $\mathrm{C}^{*}$-algebras, it does not have the injectivity property that min possesses. However it is projective as discussed in [16]. Another important aspect of the maximal tensor product is the following duality property given by Lance in [29]: A linear map $f: \mathcal{S} \otimes_{\max } \mathcal{T} \rightarrow \mathbb{C}$ is positive if and only if the corresponding map $\varphi_{f}: \mathcal{S} \rightarrow \mathcal{T}^{d}$ is completely positive. Here $\varphi_{f}(s)$ is the linear functional on $T$ given by $\varphi_{f}(s)(t)=f(s \otimes t)$. (See also [22, Lem. 5.7 and Thm. 5.8].) Consequently we obtain the following representation of the maximal tensor product:

$$
(\mathcal{S} \otimes \max \mathcal{T})^{d,+}=C P\left(\mathcal{S}, T^{d}\right) .
$$

The following property of the maximal tensor product will be useful:

Proposition 3.1. Let $\mathcal{S}_{i}$ and $\mathcal{T}_{i}$ be operator systems and $\varphi_{i}: \mathcal{S}_{i} \rightarrow \mathcal{T}_{i}$ be completely positive maps for $i=1,2$. Then the associated map $\varphi_{1} \otimes \varphi_{2}: \mathcal{S}_{1} \otimes_{\max } \mathcal{S}_{2} \rightarrow \mathcal{T}_{1} \otimes_{\max } \mathcal{T}_{2}$ is cp.

Proof. It is elementary to show that $\left(\varphi_{1} \otimes \varphi_{2}\right)^{n}\left(D_{n}^{\max }\left(\mathcal{S}_{1}, \mathcal{S}_{2}\right)\right) \subset D_{n}^{\max }\left(\mathcal{T}_{1}, \mathcal{T}_{2}\right)$. So suppose $u$ is in $C_{n}^{\max }\left(\mathcal{S}_{1}, \mathcal{S}_{2}\right)$. For any $r>0, r\left(e_{1} \otimes e_{2}\right)_{n}+u \in D_{n}^{\max }\left(\mathcal{S}_{1}, \mathcal{S}_{2}\right)$. This means that, for every $r>0, r\left(\varphi_{1}\left(e_{1}\right) \otimes \varphi_{2}\left(e_{2}\right)\right)_{n}+\left(\varphi_{1} \otimes \varphi_{2}\right)^{n}(u)$ is in $D_{n}^{\max }\left(\mathcal{T}_{1}, \mathcal{T}_{2}\right)$. Now, we can complete the positive elements $\varphi_{1}\left(e_{1}\right)$ and $\varphi_{2}\left(e_{2}\right)$ to a multiple of the units, that is, we can find positive elements $x \in \mathcal{S}_{2}$ and $y \in \mathcal{T}_{2}$ such that $\varphi_{1}\left(e_{1}\right)+x$ and $\varphi_{2}\left(e_{2}\right)+y$ are multiple of the units. Since $r(x \otimes y)_{n}$ belongs to $D_{n}^{\max }\left(\mathcal{T}_{1}, \mathcal{T}_{2}\right)$ we have that sum of these terms

$$
r(x \otimes y)_{n}+r\left(\varphi_{1}\left(e_{1}\right) \otimes \varphi_{2}\left(e_{2}\right)\right)_{n}+\left(\varphi_{1} \otimes \varphi_{2}\right)^{n}(u)=r k\left(e_{1} \otimes e_{2}\right)_{n}+\left(\varphi_{1} \otimes \varphi_{2}\right)^{n}(u)
$$

is in $D_{n}^{\max }\left(\mathcal{T}_{1}, \mathcal{T}_{2}\right)$ for every $r>0$. Thus, $\left(\varphi_{1} \otimes \varphi_{2}\right)^{n}(u) \in C_{n}^{\max }\left(\mathcal{T}_{1}, \mathcal{T}_{2}\right)$.

\subsection{Maximal Commuting Tensor Product.}

Another important tensor product we want to discuss is the maximal commuting (or commuting) tensor product which is denoted by c. It agrees with the $\mathrm{C}^{*}$-algebraic maximal tensor products on the category of unital $\mathrm{C}^{*}$-algebras however it is different then max for general operator systems. The matrix order structure is defined by using the ucp maps with commuting ranges. More precisely, if $\mathcal{S}$ and $\mathcal{T}$ are two operator systems then $C_{n}^{\text {com }}$ consist of all $\left(u_{i j}\right) \in M_{n}(\mathcal{S} \otimes \mathcal{T})$ with the property that for any Hilbert space $H$, any ucp $\phi: \mathcal{S} \rightarrow B(H)$ and $\psi: \mathcal{T} \rightarrow B(H)$ with commuting ranges

$$
(\phi \cdot \psi)^{(n)}\left(u_{i j}\right) \geq 0
$$

where $\phi \cdot \psi: \mathcal{S} \otimes \mathcal{T} \rightarrow B(H)$ is the map defined by $\phi \cdot \psi(s \otimes t)=\phi(s) \psi(t)$. The matricial cone structure $\left\{C_{n}^{c o m}\right\}$ satisfies the axioms (1), (2) and (3), and the resulting operator system is denoted by $\mathcal{S} \otimes_{c} \mathcal{T}$. The commuting tensor product $c$ is functorial and symmetric however we don't know whether is it associative or not. Before listing the main results concerning the tensor product $\mathrm{c}$ we underline the following fact: If $\tau$ is an operator system structure on $\mathcal{S} \otimes \mathcal{T}$ such that $\mathcal{S} \otimes_{\mathcal{\tau}} \mathcal{T}$ attains a representation in a $B(H)$ with " $\mathcal{S}$ " and " $\mathcal{T}$ " portions are 
commuting then $\tau \leq c$. This directly follows from the definition of $\mathrm{c}$ and justifies the name "maximal commuting". The following are Theorems 6.4 and 6.7 from [22].

Theorem 3.2. If $\mathcal{A}$ is a unital $C^{*}$-algebra and $\mathcal{S}$ is an operator system, then

$$
\mathcal{A} \otimes_{c} \mathcal{S}=\mathcal{A} \otimes_{\max } \mathcal{S}
$$

Theorem 3.3. Let $\mathcal{S}$ and $\mathcal{T}$ are operator systems. Then $\mathcal{S} \otimes_{c} \mathcal{T} \subset C_{u}^{*}(\mathcal{S}) \otimes_{\max } C_{u}^{*}(\mathcal{T})$.

In fact the following improvement of this theorem will be more useful in later sections.

Proposition 3.4. Let $\mathcal{S}$ and $\mathcal{T}$ be operator systems. Then $\mathcal{S} \otimes_{c} \mathcal{T} \subset C_{u}^{*}(\mathcal{S}) \otimes_{\max } \mathcal{T}$.

Proof. By using the functoriality of $\mathrm{c}$ we have that the following maps

$$
\mathcal{S} \otimes_{c} \mathcal{T} \stackrel{i \otimes i d}{\longrightarrow} C_{u}^{*}(\mathcal{S}) \otimes_{\max } \mathcal{T} \stackrel{i d \otimes i}{\longrightarrow} C_{u}^{*}(\mathcal{S}) \otimes_{\max } C_{u}^{*}(\mathcal{T}),
$$

where $i d$ is the identity and $i$ is the inclusion, are ucp. Theorem 3.3 ensures that the composition is a complete order embedding so the first map, which is unital, has the same property. (Here we use the fact that if the composition of two ucp maps is a complete order embedding then the first map has the same property.)

Following result is direct consequence of ([21, Cor. 6.5]) which characterizes the ucp map defined by the commuting tensor product of two operator systems:

Proposition 3.5. Let $\mathcal{S}$ and $\mathcal{T}$ be two operator systems and let $\varphi: \mathcal{S} \otimes_{c} \mathcal{T} \rightarrow B(H)$ be a ucp map. Then there is Hilbert space $K$ containing $H$ as a Hilbert subspace and ucp maps $\phi: \mathcal{S} \rightarrow B(K)$ and $\psi: \mathcal{T} \rightarrow B(K)$ with commuting ranges such that $\varphi=\left.P_{H} \phi \cdot \psi\right|_{H}$. Conversely, every such map is ucp.

\subsection{Some Asymmetric Tensor Products.}

In this subsection we discuss the enveloping left (el) and enveloping right (er) tensor products. Given operator systems $\mathcal{S}$ and $\mathcal{T}$ we define

$$
\mathcal{S} \otimes_{e l} \mathcal{T}: \subseteq I(\mathcal{S}) \otimes_{\max } \mathcal{T} \text { and } \mathcal{S} \otimes_{e r} \mathcal{T}: \subseteq \mathcal{S} \otimes_{\max } I(\mathcal{T})
$$

where $I(\cdot)$ is the injective envelope of an operator system. Both el and er are functorial tensor products. We don't know whether these tensor products are associative. They are not symmetric but asymmetric in the sense that

$$
\mathcal{S} \otimes_{e l} \mathcal{T}=\mathcal{T} \otimes_{e r} \mathcal{S} \quad \text { via the map } \quad s \otimes t \mapsto t \otimes s .
$$

el and er have the following one sided injectivity property [22, Thm. 7.5]

Theorem 3.6. The tensor product el is the maximal left injective functorial tensor product, that is, for any $\mathcal{S} \subset \mathcal{S}_{1}$ and $\mathcal{T}$ we have

$$
\mathcal{S} \otimes_{e l} \mathcal{T} \subseteq \mathcal{S}_{1} \otimes_{e l} \mathcal{T}
$$

and it is the maximal functorial tensor product with this property.

Likewise, er is the maximal right injective tensor product. It directly follows from the definition that if $\mathcal{S}$ is an injective operator system then $\mathcal{S} \otimes_{e l} \mathcal{T}=\mathcal{S} \otimes_{\max } \mathcal{T}$ for every operator system $\mathcal{T}$. Now for an arbitrary operator system $\mathcal{S}$ this allows us to conclude that the tensor product el is independent of the the injective object that we represent $\mathcal{S}$, that is, if $\mathcal{S} \hookrightarrow \mathcal{S}_{1}$ where $\mathcal{S}_{1}$ is injective then for any operator system $\mathcal{T}$, the tensor product on $\mathcal{S} \otimes \mathcal{T}$ arising from the inclusion $\mathcal{S}_{1} \otimes_{\max } \mathcal{T}$ coincides with el. To see this we only need to use the left injectivity of el:

$$
\mathcal{S} \otimes_{e l} \mathcal{T} \hookrightarrow \mathcal{S}_{1} \otimes_{e l} \mathcal{T}=\mathcal{S}_{1} \otimes_{\max } \mathcal{T}
$$


A similar property for the tensor product er holds. el and er, in general, are not comparable however they both lie between min and c.

\section{Characterization of Various Nuclearities}

In the previous section we have reviewed the tensor products in the category of operator systems. In this section we will overview the behavior of the operator systems under tensor products. More precisely, we will see several characterizations of the operator systems that fix a pair of tensor products.

Given two tensor products $\tau_{1} \leq \tau_{2}$, an operator systems $\mathcal{S}$ is said to be $\left(\tau_{1}, \tau_{2}\right)$-nuclear provided $\mathcal{S} \otimes_{\tau_{1}} \mathcal{T}=\mathcal{S} \otimes_{\tau_{2}} \mathcal{T}$ for every operator system $\mathcal{T}$. We remark that the place of the operator system $\mathcal{S}$ is important as not all the tensor products are symmetric.

\subsection{Completely Positive Factorization Property (CPFP).}

We want to start with a discussion on the characterization of (min,max)-nuclearity given in [17. An operator system $\mathcal{S}$ is said to have $C P F P$ if there is net of ucp maps

$$
\phi_{\alpha}: \mathcal{S} \rightarrow M_{n_{\alpha}} \text { and } \psi_{\alpha}: M_{n_{\alpha}} \rightarrow \mathcal{S}
$$

such that the identity $i d: \mathcal{S} \rightarrow \mathcal{S}$ approximated by $\psi_{\alpha} \circ \phi_{\alpha}$ in point-norm topology, that is, for any $s \in \mathcal{S}, \psi_{\alpha} \circ \phi_{\alpha}(s) \rightarrow s$. The following is Corollary 3.2 of [17].

Theorem 4.1. The following are equivalent for an operator system $\mathcal{S}$ :

(1) $\mathcal{S}$ is (min,max)-nuclear, that is, $\mathcal{S} \otimes_{\min } \mathcal{T}=\mathcal{S} \otimes_{\max } \mathcal{T}$ for all $\mathcal{T}$.

(2) $\mathcal{S}$ has $C P F P$.

The characterization in this theorem extends the characterization of nuclear unital $\mathrm{C}^{*}$ algebras. Recall that a unital $\mathrm{C}^{*}$-algebra $\mathcal{A}$ is said to be nuclear if $\mathcal{A} \otimes_{\min } \mathcal{B}=\mathcal{A} \otimes_{\max } \mathcal{B}$ for every $\mathrm{C}^{*}$-algebra $\mathcal{B}$. By using Proposition 3.4, it is elementary to show that $\mathcal{A}$ is nuclear if and only if it is (min,max)-nuclear operator system. Consequently the above result extends a well known result of Choi and Effros [7]. We also remark that in [24] and [27] an operator system is defined as nuclear if it satisfies CPFP. Consequently the classical term "nuclearity" coincides with the (min,max)-nuclearity.

\subsection{Operator System Local Lifting Property (osLLP).}

Another aspect we want to discuss is the operator system local lifting property (osLLP) and we will see that it is equivalent to (min,er)-nuclearity. An operator system $\mathcal{S}$ is said to have osLLP if for every unital $\mathrm{C}^{*}$-algebra $\mathcal{A}$ and ideal $I$ in $\mathcal{A}$ and for every ucp map $\varphi: \mathcal{S} \rightarrow \mathcal{A} / \mathcal{I}$ the following holds: For every finite dimensional operator subsystem $\mathcal{S}_{0}$ of $\mathcal{S}$, the restriction of $\varphi$ on $\mathcal{S}_{0}$, say $\varphi_{0}$, lifts to a completely positive map on $\mathcal{A}$ so that the following diagram commutes.

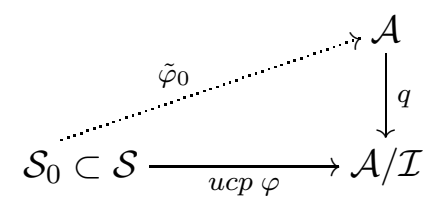

Of course, $\mathcal{S}$ may possess osLLP without a global lifting. We also remark that the completely positive local liftings can also be chosen to be ucp in the definition of osLLP (see the discussion in [21, Sec. 8]). The LLP definition for a $\mathrm{C}^{*}$-algebra given in [25] is the same. So it follows that a unital $\mathrm{C}^{*}$-algebra has LLP (in the sense of Kirchberg) if and only if it has osLLP. The following result is from [25]. 
Theorem 4.2 (Kirchberg). The following are equivalent for a $C^{*}$-algebra $\mathcal{A}$ :

(1) $\mathcal{A}$ has $L L P$

(2) $\mathcal{A} \otimes_{\min } B(H)=\mathcal{A} \otimes_{\max } B(H)$ for every Hilbert space $H$.

Here is the operator system variant given in [21]:

Theorem 4.3. The following are equivalent for an operator system $\mathcal{S}$ :

(1) $\mathcal{S}$ has osLLP.

(2) $\mathcal{S} \otimes_{\min } B(H)=\mathcal{S} \otimes_{\max } B(H)$ for every Hilbert space $H$.

(3) $\mathcal{S}$ is (min,er)-nuclear, that is, $\mathcal{S} \otimes_{\min } \mathcal{T}=\mathcal{S} \otimes_{\text {er }} \mathcal{T}$ for every $\mathcal{T}$.

It is not hard to show that in the above theorem "every Hilbert space" can be replaced by $l^{2}(\mathbb{N})$. If we denote $\mathbb{B}=B\left(l^{2}(\mathbb{N})\right)$, the above equivalent conditions, in some similar context, is also called $\mathbb{B}$-nuclearity. (See [3], e.g.) Consequently for operator systems osLLP, $\mathbb{B}$-nuclearity and (min,er)-nuclearity are all equivalent.

Remark 4.4. The definition of LLP of a $\mathrm{C}^{*}$-algebra in [41, Chp. 16 ] is different, it requires completely contractive liftings from finite dimensional operator subspaces. However, as it can be seen in [41, Thm. 16.2], all the approaches coincide for $\mathrm{C}^{*}$-algebras.

Note: When we work with the finite dimensional operator systems we remove the extra word "local", we even remove "os" and simply say "lifting property".

It seems to be important to remark that in the definition of osLLP one can can replace ucp maps by cp maps.

Remark 4.5. The following are equivalent for an operator system $\mathcal{S}$ :

(1) $\mathcal{S}$ has osLLP.

(2) For every unital $\mathrm{C}^{*}$-algebra $\mathcal{A}$ and ideal $I$ and for every cp map $\varphi: \mathcal{S} \rightarrow \mathcal{A} / I$, the restriction of $\varphi$ on any finite dimensional operator subsystem $\mathcal{S}$ has a cp lift on $\mathcal{A}$.

Proof. (2) implies (1) is clear. Conversely suppose (1) holds. This implies that $\mathcal{S} \otimes_{\min } B(H)=$ $\mathcal{S} \otimes_{\max } B(H)$. Let $\varphi: \mathcal{S} \rightarrow \mathcal{A} / I$ be a cp map and $\mathcal{S}_{0}$ is finite dimensional operator subsystem of $\mathcal{S}$. Now if we represent $\mathcal{S}_{0}^{d}$ in to a $B(H)$ (and set $\mathbb{B}=B(H)$ ) we have that

$$
\mathcal{S}_{0}^{d} \otimes_{\min } \mathcal{S} \subset \mathbb{B} \otimes_{\min } \mathcal{S}=\mathbb{B} \otimes_{\max } \mathcal{S} \stackrel{i d \otimes \varphi}{\longrightarrow} \mathbb{B} \otimes_{\max } \mathcal{A} / I
$$

is cp map where we use the injectivity of minimal tensor product and Proposition 3.1. By using first remark in Chp. 17 [41] and [21, Cor. 5.16], we have that

$$
\mathbb{B} \otimes_{\max } \mathcal{A} / I=\frac{\mathbb{B} \otimes_{\max } \mathcal{A}}{\mathbb{B} \otimes_{\max } I} \rightarrow \frac{\mathbb{B} \otimes_{\min } \mathcal{A}}{\mathbb{B} \otimes_{\min } I} \supset \frac{\mathcal{S}_{0}^{d} \otimes_{\min } \mathcal{A}}{\mathcal{S}_{0}^{d} \otimes I} .
$$

Since the inclusion $i: \mathcal{S}_{0} \rightarrow \mathcal{S}$ is cp, this corresponds to a positive element $u_{i}$ in $\mathcal{S}_{0}^{d} \otimes_{\min } \mathcal{S}$. (See [21, Lem. 8.4].) Thus, under the composition of the above maps, the image $v$ of $u_{i}$ is still positive in $\left(\mathcal{S}_{0}^{d} \otimes_{\min } \mathcal{A}\right) /\left(\mathcal{S}_{0}^{d} \otimes I\right)$. Since this quotient is proximinal (see [21, Cor. 5.15]), there is a positive element $w$ in $\mathcal{S}_{0}^{d} \otimes_{\min } \mathcal{A}$ giving $v$ under the quotient map. Now, again by using [21, Lem. 8.4.], $w$ corresponds to a cp map $\tilde{\varphi}: \mathcal{S}_{0} \rightarrow \mathcal{A}$. It is easy to verify that $\tilde{\varphi}$ is a lift of $\varphi$ when restricted to $\mathcal{S}_{0}$.

\subsection{Weak Expectation Property (WEP).}

If $\mathcal{A}$ is a unital $\mathrm{C}^{*}$-algebra then the bidual $\mathrm{C}^{*}$-algebra $\mathcal{A}^{* *}$ is unitally completely order isomorphic to the bidual operator system $\mathcal{A}^{d d}$. This allows one to extend the notion of WEP, which is introduced and shown to be a fundamental nuclearity property by Lance in [28], 
to the category of operator systems. We say that an operator system $\mathcal{S}$ has $W E P$ if the canonical inclusion $i: \mathcal{S} \hookrightarrow \mathcal{S}^{d d}$ extends to a ucp map on the injective envelope $I(\mathcal{S})$.

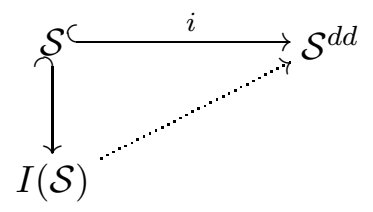

In 21] it was shown that WEP implies (el,max)-nuclearity and the difficult converse is shown in [16]. Consequently we have that

Theorem 4.6. An operator system has WEP if and only if it is (el,max)-nuclear.

\subsection{Double Commutant Expectation Property (DCEP).}

Another nuclearity property we want to discuss is DCEP which coincides with WEP for unital $\mathrm{C}^{*}$-algebras however is different than WEP for general operator systems. An operator system $\mathcal{S}$ is said to have $D C E P$ if every representation $i: \mathcal{S} \hookrightarrow B(H)$ extends to a ucp map from $I(\mathcal{S})$ into $\mathcal{S}^{\prime \prime}$, the double commutant of $\mathcal{S}$ in $B(H)$.

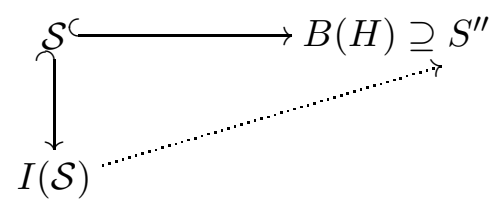

In fact, by using Arveson's commutant lifting theorem [1] (or [33, Thm. 12.7]), it can be directly shown that a unital $\mathrm{C}^{*}$-algebra has WEP if and only if it has DCEP. Many fundamental results and conjectures concerning WEP in $\mathrm{C}^{*}$-algebras reduces to DCEP in operator systems. The following is a direct consequence of Theorem 7.1 and 7.6 in [21]:

Theorem 4.7. The following are equivalent for an operator system $\mathcal{S}$ :

(1) $\mathcal{S}$ is (el,c)-nuclear, that is, $\mathcal{S} \otimes_{e l} \mathcal{T}=\mathcal{S} \otimes_{c} \mathcal{T}$ for every $\mathcal{T}$.

(2) $\mathcal{S}$ has DCEP.

(3) $\mathcal{S} \otimes_{\min } C^{*}\left(\mathbb{F}_{\infty}\right)=\mathcal{S} \otimes_{\max } C^{*}\left(\mathbb{F}_{\infty}\right)$.

(4) For any $\mathcal{S} \subset \mathcal{A}$ and $\mathcal{B}$, where $\mathcal{A}$ and $\mathcal{B}$ are unital $C^{*}$-algebras, the inclusion $\mathcal{S} \otimes_{\text {max }}$ $\mathcal{B} \hookrightarrow \mathcal{A} \otimes_{\max } \mathcal{B}$ is a complete order embedding.

Here $C^{*}\left(\mathbb{F}_{\infty}\right)$ is the full $\mathrm{C}^{*}$-algebra of the free group on countably infinite generators $\mathbb{F}_{\infty}$. Note that (3) is Kirchberg's WEP characterization in [25] and (4) is Lance's seminuclearity in [28] for unital $\mathrm{C}^{*}$-algebras.

\subsection{Exactness.}

The importance of exactness and its connection to the tensor theory of $\mathrm{C}^{*}$-algebras ensued by Kirchberg [24], 25]. Exactness is really a categorical term and requires a correct notion of quotient theory. The operator system quotients established in 21, which we reviewed in Section 2, is used to extend the exactness to operator systems. Before starting the definition we recall a couple of results from [21]: Let $\mathcal{S}$ be an operator system, $\mathcal{A}$ be a unital $\mathrm{C}^{*}$ algebra and $I$ be an ideal in $\mathcal{A}$. Then $\mathcal{S} \bar{\otimes} I$ is a kernel in $\mathcal{S} \hat{\otimes}_{\text {min }} \mathcal{A}$ where $\hat{\otimes}_{\text {min }}$ represents the completed minimal tensor product and $\bar{\otimes}$ denotes the closure of the algebraic tensor product in the larger space. By using the functoriality of the minimal tensor product it is easy to see that the map

$$
\mathcal{S} \hat{\otimes}_{\min } \mathcal{A} \stackrel{i d \otimes q}{\longrightarrow} \mathcal{S} \hat{\otimes}_{\min }(\mathcal{A} / I)
$$


where $i d$ is the identity on $\mathcal{S}$ and $q$ is the quotient map from $\mathcal{A}$ onto $\mathcal{A} / I$, is ucp and its kernel contains $\mathcal{S} \bar{\otimes} I$. Consequently the induced map

$$
\left(\mathcal{S} \hat{\otimes}_{\min } \mathcal{A}\right) /(\mathcal{S} \bar{\otimes} I) \longrightarrow \mathcal{S} \hat{\otimes}_{\min }(\mathcal{A} / I)
$$

is still unital and completely positive. An operator system is said to be exact if this induced map is a bijective and a complete order isomorphism for every $\mathrm{C}^{*}$-algebra $\mathcal{A}$ and ideal $I$ in $\mathcal{A}$. In other words we have the equality

$$
\left(\mathcal{S} \hat{\otimes}_{\min } \mathcal{A}\right) /(\mathcal{S} \bar{\otimes} I)=\mathcal{S} \hat{\otimes}_{\min }(\mathcal{A} / I)
$$

We remark that the induced map may fail to be surjective or injective, moreover even if it has these properties it may fail to be a complete order isomorphism.

Remark 4.8. If $\mathcal{S}$ is finite dimensional then we have that $\mathcal{S} \otimes_{\min } \mathcal{A}=\mathcal{S} \hat{\otimes}_{\text {min }} \mathcal{A}$ and $\mathcal{S} \bar{\otimes} I=$ $\mathcal{S} \otimes I$. Moreover the induced map

$$
\left(\mathcal{S} \otimes_{\min } \mathcal{A}\right) /(\mathcal{S} \otimes I) \longrightarrow \mathcal{S} \otimes_{\min }(\mathcal{A} / I)
$$

is always bijective. Thus, for this case, exactness is equivalent to the statement that the induced map is a complete order isomorphism.

Proof. Let $\mathcal{S}=\operatorname{span}\left\{s_{1}, \ldots, s_{k}\right\}$. Suppose that $\left\{u_{n}\right\}$ is a Cauchy sequence in the algebraic tensor product $\mathcal{S} \otimes_{\min } \mathcal{A}$ with limit $u$ in $\mathcal{S} \hat{\otimes}_{\min } \mathcal{A}$. We will show that $u$ belongs to $\mathcal{S} \otimes_{\text {min }} \mathcal{A}$. Clearly we can write $u_{n}=s_{1} \otimes a_{1}^{n}+\cdots s_{k} \otimes a_{k}^{n}$. We will prove that $\left\{a_{i}^{n}\right\}_{n}$ is Cauchy in $\mathcal{A}$ for every $i=1, \ldots, k$. Let $\delta_{i}: \mathcal{S} \rightarrow \mathbb{C}$ be the linear map defined by $\delta_{i}\left(s_{j}\right)=\delta_{i j}$. Since each of $\delta_{i}$ is completely bounded we have that $\delta \otimes i d: \mathcal{S} \otimes_{\min } \mathcal{A} \rightarrow \mathcal{A}$ given by $s \otimes a \mapsto \delta(s) a$ is a completely bounded map, in particular it is continuous. (Here we use the fact that minimal tensor product of two operator system is same as the operator space minimal tensor product. This is easy to see as both of them are spatial. We also use the fact that every linear map defined from a finite dimensional operator space is completely bounded.) Clearly $\left\{a_{i}^{n}\right\}_{n}$ is the image of $\left\{u_{n}\right\}$ under this map and consequently it is Cauchy. Let $a_{i}$ be the limit of these sequences in $\mathcal{A}$ for $i=1, \ldots, k$. Now it is elementary to show that $u=s_{1} \otimes a_{1}+\cdots s_{k} \otimes a_{k}$. This directly follows from the triangle inequality and the cross norm property of the minimal tensor product, i.e., $\|s \otimes a\|=\|s\|\|a\|)$.

The proof of the fact that $\mathcal{S} \bar{\otimes} I=\mathcal{S} \otimes I$ is similar to this so we skip it. It is elementary to see that the image of the induced map

$$
\left(\mathcal{S} \otimes_{\min } \mathcal{A}\right) /(\mathcal{S} \otimes I) \longrightarrow \mathcal{S} \otimes_{\min }(\mathcal{A} / I)
$$

covers the algebraic quotient which is same as its completion for this case. Thus, it is onto. Finally we need to show that it is injective. More precisely, we need to show that the map $\mathcal{S} \otimes_{\min } \mathcal{A} \rightarrow \mathcal{S} \otimes \mathcal{A} / I$ has kernel $\mathcal{S} \otimes I$. Suppose the image of $\Sigma s_{i} \otimes a_{i}$ is 0 , that is, $\Sigma s_{i} \otimes \dot{a}_{i}$ is 0 in $\mathcal{S} \otimes \mathcal{A} / I$. Since $\left\{s_{1}, \ldots, s_{n}\right\}$ is a linearly independent set we have that each of $\dot{a_{1}}, \ldots, \dot{a_{k}}$ is 0 . Thus $a_{1}, \ldots, a_{k}$ belongs to $I$. This finishes the proof.

Note: The term exactness in this paper coincides with 1-exactness in [21].

A unital $\mathrm{C}^{*}$-algebra is exact (in the sense of Kirchberg) if and only if it is an exact operator system which follows from the fact that the unital $\mathrm{C}^{*}$-algebra ideal quotient coincides with the operator system kernel quotient. The following is Theorem 5.7 of [21]:

Theorem 4.9. An operator system is exact if and only if it is (min,el)-nuclear.

In Theorem 6.6 we will see that exactness and the lifting property are dual pairs. We want to finish this subsection with the following stability property: 
Proposition 4.10. Exactness passes to operator subsystems. That is, if $\mathcal{S}$ is exact then every operator subsystem of $\mathcal{S}$ is exact. Conversely, if every finite dimensional operator subsystem of $\mathcal{S}$ is exact then $\mathcal{S}$ is exact.

Proof. We will use the nuclearity characterization of exactness, i.e., (min,el)-nuclearity. First suppose $\mathcal{S}$ is exact and $\mathcal{S}_{0}$ is an operator subsystem of $\mathcal{S}$. By using the injectivity of min and left injectivity of el we have that

$$
\mathcal{S}_{0} \otimes_{\text {min }} \mathcal{T} \subseteq \mathcal{S} \otimes_{\text {min }} \mathcal{T} \text { and } \mathcal{S}_{0} \otimes_{e l} \mathcal{T} \subseteq \mathcal{S} \otimes_{e l} \mathcal{T}
$$

for every operator system $\mathcal{T}$. Since the tensors on the right hand side coincide it follows that $\mathcal{S}_{0}$ is (min,el)-nuclear, equivalently it is exact.

To prove the second part suppose that $\mathcal{S}$ is not exact. This means that there is an operator system $\mathcal{T}$ such that the identity

$$
\mathcal{S} \otimes_{\min } \mathcal{T} \rightarrow \mathcal{S} \otimes_{e l} \mathcal{T}
$$

is not a cp map, that is, there is an positive element $U$ in $M_{n}\left(\mathcal{S} \otimes_{\min } \mathcal{T}\right)$ which is not positive in $M_{n}\left(\mathcal{S} \otimes_{e l} \mathcal{T}\right)$. Clearly $\mathcal{S}$ has a finite dimensional operator subsystem $\mathcal{S}_{0}$ such that $U$ belongs to $M_{n}\left(\mathcal{S}_{0} \otimes \mathcal{T}\right)$. Now again using the fact that

$$
\mathcal{S}_{0} \otimes_{\min } \mathcal{T} \subseteq \mathcal{S} \otimes_{\min } \mathcal{T} \text { and } \mathcal{S}_{0} \otimes_{e l} \mathcal{T} \subseteq \mathcal{S} \otimes_{e l} \mathcal{T}
$$

we see that $U$ is positive in $M_{n}\left(\mathcal{S}_{0} \otimes_{\min } \mathcal{T}\right)$ but not positive in $M_{n}\left(\mathcal{S}_{0} \otimes_{e l} \mathcal{T}\right)$. This means that $\mathcal{S}_{0}$ is not exact. This finishes the proof.

\subsection{Final Remarks on Nuclearity.}

Unlike $\mathrm{C}^{*}$-algebras a finite dimensional operator system may not posses a certain type of nuclearity. For example $M_{2} \oplus M_{2}$ has a five dimensional operator subsystem which does not have the lifting property (See Corollary 10.14, e.g.). Exactness and the local lifting property of three dimensional operator systems are directly related to the Smith Ward problem which is currently still open (Sec. 11). Similarly we will see that the Kirchberg Conjecture is a problem about nuclearity properties of five dimensional operator systems.

The following schema summarizes the nuclearity characterizations that we have discussed in this section:

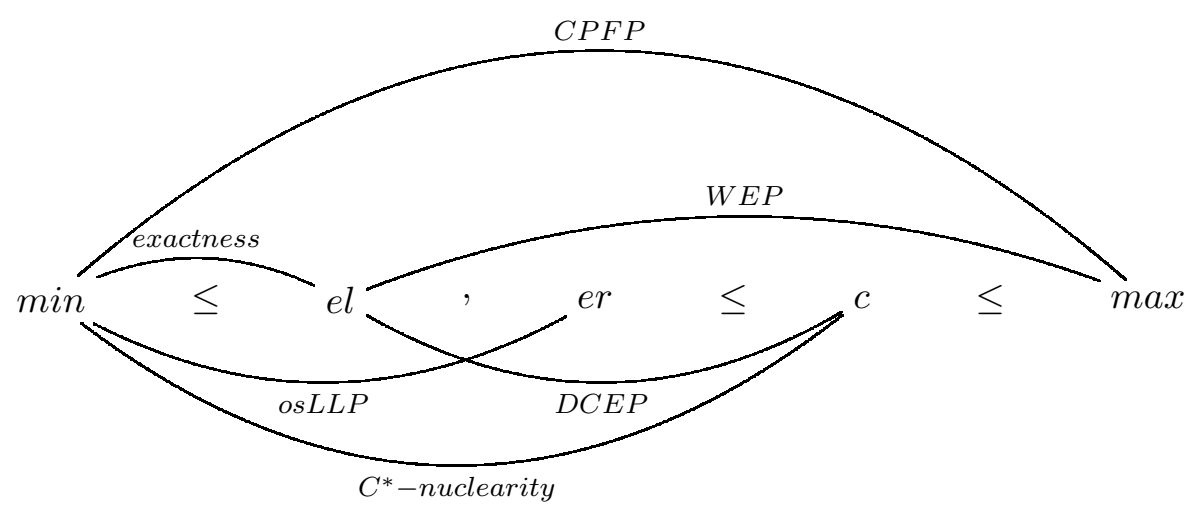

Proposition 4.11. The following are equivalent for an operator system $\mathcal{S}$ :

(1) $\mathcal{S}$ is (min,c)-nuclear, that is, $\mathcal{S} \otimes_{\min } \mathcal{T}=\mathcal{S} \otimes_{c} \mathcal{T}$ for every operator system $\mathcal{T}$.

(2) $\mathcal{S}$ is $C^{*}$-nuclear, that is, $\mathcal{S} \otimes_{\min } \mathcal{A}=\mathcal{S} \otimes_{\max } \mathcal{A}$ for every unital $C^{*}$-algebra $\mathcal{A}$. 
Proof. Suppose (1). By using Theorem 3.2 we have that $\mathcal{S} \otimes_{\min } \mathcal{A}=\mathcal{S} \otimes_{c} \mathcal{A}=\mathcal{S} \otimes_{\max } \mathcal{A}$. Hence we obtain (2). Conversely suppose (2). By the injectivity of the minimal tensor product and by Proposition 3.4 we have the inclusions

$$
\mathcal{S} \otimes_{\min } \mathcal{T} \subseteq \mathcal{S} \otimes_{\min } C_{u}^{*}(\mathcal{T}) \text { and } \mathcal{S} \otimes_{c} \mathcal{T} \subseteq \mathcal{S} \otimes_{\max } C_{u}^{*}(\mathcal{T})
$$

Since the tensor products on the right hand side coincides (1) follows.

\section{Remarks:}

(1) We use the term $\mathrm{C}^{*}$-nuclearity rather than (min,c)-nuclearity.

(2) The above table for unital $\mathrm{C}^{*}$-algebras summarizes the classical discussion for $\mathrm{C}^{*}$ algebras. Recall that in this case $c$ and max coincides and consequently WEP and DCEP are the same properties. Also osLLP and LLP are the same. It is also important to remark that if we start with a unital $\mathrm{C}^{*}$-algebra $\mathcal{A}$ then (min,el)-nuclearity, for example, can be verified with unital $\mathrm{C}^{*}$-algebras. That is, $\mathcal{A} \otimes_{\min } \mathcal{T}=\mathcal{A} \otimes_{e l} \mathcal{T}$ for every operator system $\mathcal{T}$ if and only if $\mathcal{A} \otimes_{\text {min }} \mathcal{B}=\mathcal{A} \otimes_{\text {el }} \mathcal{B}$ for every unital $\mathrm{C}^{*}$-algebra $\mathcal{B}$. We left the verification of this to the reader. In addition to this, as we pointed out before, $\mathcal{A}$ is exact (in the sense of Kirchberg) if and only if it is an exact operator system. Similar properties hold for other nuclearity properties WEP, CPFP and LLP. Thus, we obtain the following schema:

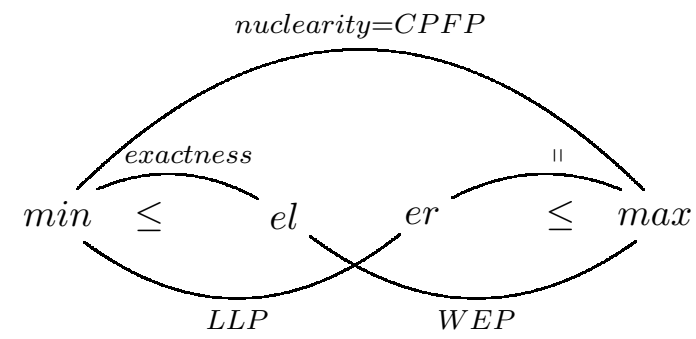

For this case (er,max)-nuclearity of a $\mathrm{C}^{*}$-algebra coincides with the nuclearity by Lance [28], (see also [22, Prop. 7.7]). By this simple schema it is rather easy to see that nuclearity is equivalent to exactness and WEP, e.g. Also suppose that $\mathcal{A}$ and $\mathcal{B}$ are unital $\mathrm{C}^{*}$-algebras such that $\mathcal{A}$ has WEP and $\mathcal{B}$ has LLP. Now by using the fact that LLP is equivalent to (min,er)-nuclearity we have that $\mathcal{A} \otimes_{\min } \mathcal{B}=\mathcal{A} \otimes_{e l} \mathcal{B}$. (Note: $\mathcal{B}$ is on the right hand side.) Again by using the fact that WEP is same as (el,c=max)-nuclearity we have $\mathcal{A} \otimes_{e l} \mathcal{B}=\mathcal{A} \otimes_{\max } \mathcal{B}$. Thus we obtain a well known result of Kirchberg: $\mathcal{A} \otimes_{\min } \mathcal{B}=\mathcal{A} \otimes_{\max } \mathcal{B}$.

We close this section with the following observation about finite dimensional operator systems. Roughly speaking it states that the finite dimensional operator systems, except a small portion, namely the $\mathrm{C}^{*}$-algebras, are never (c,max)-nuclear. So in this case, (min,c)nuclearity (i.e. $\mathrm{C}^{*}$-nuclearity) is the highest nuclearity that that one should expect. (Of course, among the tensor products $\min \leq \mathrm{el}$, er $\leq \mathrm{c} \leq \max$.)

Proposition 4.12. The following are equivalent for a finite dimensional operator system $\mathcal{S}$ :

(1) $\mathcal{S}$ is (c,max)-nuclear.

(2) $\mathcal{S}$ is unitally completely order isomorphic to a $C^{*}$-algebra.

(3) $\mathcal{S} \otimes_{c} \mathcal{S}^{d}=\mathcal{S} \otimes_{\max } \mathcal{S}^{d}$.

Proof. Since $c$ and $\max$ coincides when one of the tensorants is a $\mathrm{C}^{*}$-algebra, (2) implies (1). Clearly (1) implies (3). We will show that (3) implies (2). Consider $i d: \mathcal{S} \rightarrow \mathcal{S}$. This corresponds to a positive linear functional $f_{i d}: \mathcal{S} \otimes_{\max } \mathcal{S}^{d} \rightarrow \mathbb{C}$. Since max and $c$ coincide 
by the assumption and $\mathcal{S} \otimes_{c} \mathcal{S}^{d} \subset C_{u}^{*}(\mathcal{S}) \otimes_{\max } \mathcal{S}^{d}, f_{i d}$ extends to a positive linear functional $\tilde{f_{i d}}: C_{u}^{*}(\mathcal{S}) \otimes_{\max } \mathcal{S}^{d} \rightarrow \mathbb{C}$ by Arveson's extension theorem. Let $\varphi: C_{u}^{*}(\mathcal{S}) \rightarrow\left(S^{d}\right)^{d}=\mathcal{S}$ be the corresponding cp map. Clearly $\varphi$ extends the identity on $\mathcal{S}$. Now by using a slight modification of [33, Theorem 15.2] we have that $\mathcal{S}$ has a structure of a $\mathrm{C}^{*}$-algebra.

\section{WEP AND KirchBerg's CONJECTURE}

In this section we improve Kirchberg's WEP characterization for unital $\mathrm{C}^{*}$-algebras and we express Kirchberg's Conjecture in terms of a five dimensional operator system system problem. The last schema in the previous section still includes many question marks. There is no known example of a non-nuclear $\mathrm{C}^{*}$-algebra which has WEP and LLP. One another major open question is whether LLP implies WEP, which is known as the Kirchberg Conjecture. More precisely, in his astonishing paper [25] he proves that:

Theorem 5.1 (Kirchberg). The following are equivalent:

(1) Every separable $I I_{1}$-factor is a von Neumann subfactor of the ultrapower $R_{\omega}$ of the hyperfinite $I I_{1}$-factor $R$ for some ultrafilter $\omega \in \beta \mathbb{N} \backslash \mathbb{N}$.

(2) For a unital $C^{*}$-algebra LLP implies WEP.

(3) Every unital $C^{*}$-algebra is a quotient of a $C^{*}$-algebra that has WEP (i.e. QWEP).

(4) $C^{*}\left(\mathbb{F}_{\infty}\right) \otimes_{\min } C^{*}\left(\mathbb{F}_{\infty}\right)=C^{*}\left(\mathbb{F}_{\infty}\right) \otimes_{\max } C^{*}\left(\mathbb{F}_{\infty}\right)$.

(5) $C^{*}\left(\mathbb{F}_{\infty}\right)$ has WEP.

The equivalent conditions in this theorem are still unknown. The first one is the Connes' Embedding Problem. We refer to [9] for related definitions on this subject. The remaining equivalent arguments are known as the Kirchberg Conjecture (or Kirchberg's QWEP Conjecture). As we pointed out before $C^{*}\left(\mathbb{F}_{\infty}\right)$ (resp., $\left.C^{*}\left(\mathbb{F}_{n}\right)\right)$ stands for the full $\mathrm{C}^{*}$-algebra of the free group with a countably infinite number of (resp., with $n$ ) generators. As shown in [25], in the above theorem $C^{*}\left(\mathbb{F}_{\infty}\right)$ can be replaced by $C^{*}\left(\mathbb{F}_{2}\right)$. In fact, since there is an injective group homomorphism $\rho: \mathbb{F}_{\infty} \rightarrow \mathbb{F}_{2}$, by using Proposition 8.8. in [41], we have that $C^{*}\left(\mathbb{F}_{\infty}\right)$ can be represented as a $\mathrm{C}^{*}$-subalgebra of $C^{*}\left(\mathbb{F}_{2}\right)$ and, again by using the same theorem, there is ucp inverse of this representation. Consequently the identity on $C^{*}\left(\mathbb{F}_{\infty}\right)$ factors via ucp maps through $C^{*}\left(\mathbb{F}_{2}\right)$. Conversely, the identity on $C^{*}\left(\mathbb{F}_{2}\right)$ factors via ucp maps through $C^{*}\left(\mathbb{F}_{\infty}\right)$ in a trivial way.

Lemma 5.2. Let $\mathcal{S}$ and $\mathcal{T}$ be two operator systems. If the identity on $\mathcal{S}$ factors via ucp maps through $\mathcal{T}$ then any nuclearity property of $\mathcal{T}$ passes to $\mathcal{S}$. That is if $\mathcal{T}$ is $\left(\tau_{1}, \tau_{2}\right)$-nuclear, where $\tau_{1}$ and $\tau_{2}$ are functorial tensor products with $\tau_{1} \leq \tau_{2}$, then $\mathcal{S}$ has the same property.

Proof. Let $\phi: \mathcal{S} \rightarrow \mathcal{T}$ and $\psi: \mathcal{T} \rightarrow \mathcal{S}$ be the ucp maps such that $\psi \circ \phi(s)=s$ for every $s$ in $\mathcal{S}$. Let $\mathcal{R}$ be any operator system. Then, by using the functoriality we have that

$$
\mathcal{S} \otimes_{\tau_{1}} \mathcal{R} \stackrel{\phi \otimes i d}{\longrightarrow} \mathcal{T} \otimes_{\tau_{1}} \mathcal{R}=\mathcal{T} \otimes_{\tau_{2}} \mathcal{R} \stackrel{\psi \otimes i d}{\longrightarrow} \mathcal{S} \otimes_{\tau_{2}} \mathcal{R}
$$

is a sequence of ucp maps such that the composition is the identity. Since $\tau_{1} \leq \tau_{2}$ we have that $\mathcal{S} \otimes_{\tau_{1}} \mathcal{R}=\mathcal{S} \otimes_{\tau_{2}} \mathcal{R}$. Thus, $\mathcal{S}$ is $\left(\tau_{1}, \tau_{2}\right)$-nuclear.

Since WEP, equivalently DCEP for $\mathrm{C}^{*}$-algebras, coincides with (el,max)-nuclearity, it follows that $C^{*}\left(\mathbb{F}_{\infty}\right)$ has WEP if and only if $C^{*}\left(\mathbb{F}_{2}\right)$ has WEP. By a similar argument the above conditions are equivalent to the statement $C^{*}\left(\mathbb{F}_{2}\right) \otimes_{\min } C^{*}\left(\mathbb{F}_{2}\right)=C^{*}\left(\mathbb{F}_{2}\right) \otimes_{\max } C^{*}\left(\mathbb{F}_{2}\right)$. We also remark that Kirchberg's WEP characterization can be given as follows, which will be useful when we express WEP in terms of a tensor product with a lower dimensional operator system: 
Theorem 5.3. The following are equivalent for a unital $C^{*}$-algebra $\mathcal{A}$ :

(1) $\mathcal{A}$ has WEP.

(2) $\mathcal{A} \otimes_{\min } C^{*}\left(\mathbb{F}_{\infty}\right)=\mathcal{A} \otimes_{\max } C^{*}\left(\mathbb{F}_{\infty}\right)$.

(3) $\mathcal{A} \otimes_{\min } C^{*}\left(\mathbb{F}_{2}\right)=\mathcal{A} \otimes_{\max } C^{*}\left(\mathbb{F}_{2}\right)$.

Proof. Equivalence of (1) and (2) is the Kirchberg's WEP characterization. To see that (3) implies (2) we again use the fact that the identity on $C^{*}\left(\mathbb{F}_{\infty}\right)$ factors through ucp maps on $C^{*}\left(\mathbb{F}_{\infty}\right)$. So let $\phi: C^{*}\left(\mathbb{F}_{\infty}\right) \rightarrow C^{*}\left(\mathbb{F}_{2}\right)$ and $\psi: C^{*}\left(\mathbb{F}_{2}\right) \rightarrow C^{*}\left(\mathbb{F}_{\infty}\right)$ be the ucp maps whose composition is the identity on $C^{*}\left(\mathbb{F}_{\infty}\right)$. Now, suppose (3) holds. By using the functoriality of min and max we have that

$$
\mathcal{A} \otimes_{\min } C^{*}\left(\mathbb{F}_{\infty}\right) \stackrel{i d \otimes \phi}{\longrightarrow} \mathcal{A} \otimes_{\min } C^{*}\left(\mathbb{F}_{2}\right)=\mathcal{A} \otimes_{\max } C^{*}\left(\mathbb{F}_{2}\right) \stackrel{i d \otimes \psi}{\longrightarrow} \mathcal{A} \otimes_{\max } C^{*}\left(\mathbb{F}_{\infty}\right) .
$$

is a sequence of ucp maps such that the composition is the identity. Thus (2) holds. (2) implies (3) is similar.

Since WEP and LLP has natural extensions to general operator systems it is natural to approach Kirchberg's Conjecture from this perspective. We define $\mathcal{S}_{n}$ as the operator system in $C^{*}\left(\mathbb{F}_{n}\right)$ generated by the unitary generators, that is,

$$
\mathcal{S}_{n}=\operatorname{span}\left\{g_{1}, \ldots, g_{n}, e, g_{1}^{*}, \ldots, g_{n}^{*}\right\} \subset C^{*}\left(F_{n}\right) .
$$

$\mathcal{S}_{n}$ can also be considered as the universal operator system generated by $n$ contractions as it satisfies the following universal property: Every function $f:\left\{g_{i}\right\}_{i=1}^{n} \rightarrow \mathcal{T}$ with $\left\|f\left(g_{i}\right)\right\| \leq 1$ extends uniquely to a ucp map $\varphi_{f}: S_{n} \rightarrow \mathcal{T}$ (in an obvious way).

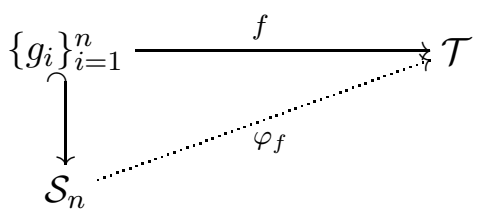

The proof this property relies on the unitary dilation of a contraction and the reader may refer to the discussion in [21, Sec. 9]. From this one can easily deduce that $\mathcal{S}_{n}$ has the lifting property. Indeed, let $\varphi: \mathcal{S}_{n} \rightarrow \mathcal{A} / I$ is a ucp map where $I \subset \mathcal{A}$ is an ideal, unital $\mathrm{C}^{*}$-algebra couple. Let $\varphi\left(g_{i}\right)=a_{i}+I$ for $i=1, \ldots, n$. Since $\mathrm{C}^{*}$-algebra ideal quotients are proximinal (see [1, Lem. 2.4.6.] e.g.) there exists $b_{i}$ in $\mathcal{A}$ such that $b_{i}+I=a_{i}+I$ with $\left\|b_{i}\right\|=\left\|a_{i}+I\right\|$. Since a ucp map is contractive we have that $\left\|a_{i}+I\right\| \leq 1$ and so $\left\|b_{i}\right\| \leq 1$. Therefore the function $g_{i} \mapsto b_{i}$ extends uniquely to a ucp map. It is elementary to show that this map is a lift of $\varphi$.

An operator subsystem $\mathcal{S}$ of a $\mathrm{C}^{*}$-algebra $\mathcal{A}$ is said to contain enough unitaries if there is a collection of unitaries in $\mathcal{S}$ which generates $\mathcal{A}$ as a $\mathrm{C}^{*}$-algebra, that is, $\mathcal{A}$ is the smallest $\mathrm{C}^{*}$-algebra that contains these unitaries. This notion is inspired by a work of Pisier (see Chp. 13 of [41]) and in [21] it was shown that several nuclearity properties of $\mathcal{A}$ can be deduced from $\mathcal{S}$ (see [21, Cor. 9.6]).

Lemma 5.4. Let $\mathcal{A}$ and $\mathcal{B}$ be unital $C^{*}$-algebras and $\left\{u_{\alpha}\right\}$ be a collection of unitaries in $\mathcal{A}$ which generates $\mathcal{A}$ as a $C^{*}$-algebra. If $\varphi: \mathcal{A} \rightarrow \mathcal{B}$ is a ucp map such that $\varphi\left(u_{\alpha}\right)$ is a unitary in $\mathcal{B}$ for every $\alpha$ then $\varphi$ is a $*$-homomorphism.

Proof. This is an application of Choi's work on the multiplicative domains in [5]. Since $e=\varphi\left(u_{\alpha} u_{\alpha}^{*}\right)=\varphi\left(u_{\alpha}\right) \varphi\left(u_{\alpha}\right)^{*}=\varphi\left(u_{\alpha}^{*} u_{\alpha}\right)=\varphi\left(u_{\alpha}\right)^{*} \varphi\left(u_{\alpha}\right)$, each $u_{\alpha}$ belongs to multiplicative domain of $\varphi$. These elements generates $\mathcal{A}$, thus, $\varphi$ is a $*$-homomorphism. 
Lemma 5.5. Let $\mathcal{S} \subset \mathcal{A}$ contain enough unitaries and let $\mathcal{B}$ be a unital $C^{*}$-algebra. Let $\left\{u_{\alpha}\right\}$ be the collection of unitaries in $\mathcal{S}$ which generates $\mathcal{A}$. Suppose $\varphi: \mathcal{S} \rightarrow \mathcal{B}$ is a ucp map such that $\varphi\left(u_{\alpha}\right)$ is a unitary in $\mathcal{B}$ for every $\alpha$. Then $\varphi$ extends uniquely to a ucp map on $\mathcal{A}$ which is necessarily a *-homomorphism.

Proof. Lemma 4.16 in [21] ensures that $\varphi$ extends to a $*$-homomorphism. So there exists a ucp extension of $\varphi$ on $\mathcal{A}$. Also the above lemma implies that any ucp extension has to be a *-homomorphism. Since $\left\{u_{\alpha}\right\}$ generates $\mathcal{A}$ and every extension coincides on $\left\{u_{\alpha}\right\}$ it follows that extension is unique.

Proposition 5.6. Suppose $\mathcal{S} \subset \mathcal{A}$ contains enough unitaries. Then $\mathcal{A}$ coincides with the enveloping $C^{*}$-algebra of $\mathcal{S}$, that is, the unique unital $*$-homomorphism $\pi: \mathcal{A} \rightarrow C_{e}^{*}(\mathcal{S})$ which extends the inclusion of $\mathcal{S}$ in $C_{e}^{*}(\mathcal{S})$ is bijective.

Proof. Let $\left\{u_{\alpha}\right\}$ be the collection of unitaries in $\mathcal{S}$ which generates $\mathcal{A}$ as a $\mathrm{C}^{*}$-algebra. Let $i$ be the inclusion of $\mathcal{S}$ in $C_{e}^{*}(\mathcal{S})$. Note that the image $\left\{\pi\left(u_{\alpha}\right)=i\left(u_{\alpha}\right)\right\}$ of the unitary collection $\left\{u_{\alpha}\right\}$ form a set of unitaries and it generates the image of $\pi$ which coincides with $C_{e}^{*}(\mathcal{S})$. We can represent $\mathcal{A}$ into a $B(H)$ as a $\mathrm{C}^{*}$-subalgebra. Now, by Arveson's extension theorem, the inclusion of $\mathcal{S}$ in $\mathcal{A} \subset B(H)$ extends to ucp map $\varphi$ on $C_{e}^{*}(\mathcal{S})$. Note that $\varphi\left(i\left(u_{\alpha}\right)\right)=u_{\alpha}$, that is, $\varphi$ maps a collection of unitaries, which generates $C_{e}^{*}(\mathcal{S})$, to a collection of unitaries in $B(H)$. Now by using the above lemma $\varphi$ must be a unital $*$-homomorphism. Moreover, since the image of $\left\{i\left(u_{\alpha}\right)\right\}$ stays in $\mathcal{A}$ and generates $\mathcal{A}$, the image of $\varphi$ is precisely $\mathcal{A}$. The rigidity of the enveloping $\mathrm{C}^{*}$-algebra ensures that $\varphi$ is one to one too. Note that $\varphi^{-1}$ is again a unital $*$-homomorphism such that $\varphi^{-1}(s)=i(s)$ for every $s$ in $\mathcal{S}$. Now the universal property of the enveloping $\mathrm{C}^{*}$-algebras ensure that $\pi=\varphi^{-1}$, thus $\pi$ is bijective.

Despite this result we still prefer to use the term "contains enough unitaries". Our very first example is, of course, $\mathcal{S}_{n} \subset C^{*}\left(\mathbb{F}_{n}\right)$. This also means that $C_{e}^{*}\left(\mathcal{S}_{n}\right)=C^{*}\left(\mathbb{F}_{n}\right)$. It is also important to remark that not every operator system contains enough unitaries in its enveloping $\mathrm{C}^{*}$-algebra. The following is an improvement of Proposition 9.5 of [21]:

Proposition 5.7. Suppose $\mathcal{S} \subset \mathcal{A}$ and $\mathcal{T} \subset \mathcal{B}$ contains enough unitaries. Then

$$
\mathcal{S} \otimes_{\min } \mathcal{T} \subset \mathcal{A} \otimes_{\max } \mathcal{B} \Longrightarrow \mathcal{A} \otimes_{\min } \mathcal{B}=\mathcal{A} \otimes_{\max } \mathcal{B}
$$

Proof. Let $\left\{u_{\alpha}\right\}$ and $\left\{v_{\beta}\right\}$ be unitaries in $\mathcal{S}$ and $\mathcal{T}$ that generates $\mathcal{A}$ and $\mathcal{B}$, respectively. By using the injectivity of the minimal tensor product we have the inclusion $\mathcal{S} \otimes_{\text {min }} \mathcal{T} \subset \mathcal{A} \otimes_{\text {min }} \mathcal{B}$. It is not hard to see that the unitaries $\left\{u_{\alpha} \otimes v_{\beta}\right\}$, which belongs to $\mathcal{S} \otimes_{\min } \mathcal{T}$, generates $\mathcal{A} \otimes_{\min } \mathcal{B}$. It is also clear that the inclusion $\mathcal{S} \otimes_{\min } \mathcal{T} \hookrightarrow \mathcal{A} \otimes_{\max } \mathcal{B}$ maps these unitaries to unitaries again. Thus, by Lemma [5.5, this inclusion extends uniquely to a $*$-homomorphism which is necessarily the identity. So we conclude that $\mathcal{A} \otimes_{\min } \mathcal{B}=\mathcal{A} \otimes_{\max } \mathcal{B}$.

Corollary 5.8. Suppose $\mathcal{S} \subset \mathcal{A}$ and $\mathcal{T} \subset \mathcal{B}$ contains enough unitaries. Then

$$
\mathcal{S} \otimes_{\min } \mathcal{T}=\mathcal{S} \otimes_{c} \mathcal{T} \Longrightarrow \mathcal{A} \otimes_{\min } \mathcal{B}=\mathcal{A} \otimes_{\max } \mathcal{B} .
$$

Proof. Let $\mathcal{S} \otimes_{\tau} \mathcal{T}$ be the operator system tensor product arising from the inclusion $\mathcal{A} \otimes_{\max } \mathcal{B}$. Clearly min $\leq \tau \leq$ c. (Note: $\mathrm{c}$ is the maximal commuting tensor product.) Since min and c coincides on $\mathcal{S} \otimes \mathcal{T}$ we have that $\mathcal{S} \otimes_{\min } \mathcal{T} \subset \mathcal{A} \otimes_{\max } \mathcal{B}$. Thus, by Proposition 5.7, the result follows.

Theorem 5.9. The following are equivalent for a unital $C^{*}$-algebra $\mathcal{A}$ :

(1) $\mathcal{A}$ has WEP (equivalently DCEP).

(2) $\mathcal{A} \otimes_{\min } \mathcal{S}_{2}=\mathcal{A} \otimes_{\max } \mathcal{S}_{2}$. 
Proof. We already know that WEP and DCEP are equivalent for $\mathrm{C}^{*}$-algebras. Now suppose (1). Since WEP coincides with (el,max)-nuclearity and $\mathcal{S}_{2}$ has the lifting property (equivalently (min,er)-nuclearity) (also keeping in mind that it is written on the right hand side) we have

$$
\mathcal{A} \otimes_{\min } \mathcal{S}_{2}=\mathcal{A} \otimes_{\text {el }} \mathcal{S}_{2}=\mathcal{A} \otimes_{\max } \mathcal{S}_{2} .
$$

Conversely suppose (2) holds. Since $\mathcal{S}_{2}$ contains enough unitaries in $C^{*}\left(\mathbb{F}_{2}\right)$ (and $\mathcal{A}$ contains enough unitaries in itself), by the above corollary, we obtain that $\mathcal{A} \otimes_{\min } C^{*}\left(\mathbb{F}_{2}\right)=\mathcal{A} \otimes_{\max }$ $C^{*}\left(\mathbb{F}_{2}\right)$. Thus $\mathcal{A}$ has WEP.

In the following theorem the equivalence of (1)-(4) is Theorem 9.1. and 9.4 of [21]. So we will only prove that these are equivalent to (5) and (6), which express $\mathrm{KC}$ in terms of a five dimensional operator system problem.

Theorem 5.10. The following are equivalent:

(1) Kirchberg Conjecture has an affirmative answer.

(2) $\mathcal{S}_{n}$ has DCEP for every $n$.

(3) $\mathcal{S}_{n} \otimes_{\min } \mathcal{S}_{n}=\mathcal{S}_{n} \otimes_{c} \mathcal{S}_{n}$ for every $n$.

(4) Every finite dimensional operator system with the lifting property has DCEP.

(5) $\mathcal{S}_{2}$ has DCEP.

(6) $\mathcal{S}_{2} \otimes_{\min } \mathcal{S}_{2}=\mathcal{S}_{2} \otimes_{c} \mathcal{S}_{2}$

Proof. The equivalence of (1),(2),(3) and (4) follows from Theorem 9.1 and 9.4 of [21]. These conditions clearly imply (5) and (6). Moreover (5) implies (6). In fact, we know that $\mathcal{S}_{2}$ has the lifting property (equivalently (min,er)-nuclearity). If we assume that it has DCEP (equivalently (el,c)-nuclearity) then (also keeping in mind that one of the $\mathcal{S}_{2}$ is written on the right hand side) it follows that

$$
\mathcal{S}_{2} \otimes_{\min } \mathcal{S}_{2}=\mathcal{S}_{2} \otimes_{e r} \mathcal{S}_{2}=\mathcal{S}_{2} \otimes_{c} \mathcal{S}_{2}
$$

Conversely suppose that (6) holds. Since $\mathcal{S}_{2}$ contains enough unitaries in $C^{*}\left(\mathbb{F}_{2}\right)$, by Corollary 5.8 , it follows that $C^{*}\left(\mathbb{F}_{2}\right) \otimes_{\min } C^{*}\left(\mathbb{F}_{2}\right)=C^{*}\left(\mathbb{F}_{2}\right) \otimes_{\max } C^{*}\left(\mathbb{F}_{2}\right)$, that is, the Kirchberg Conjecture has an affirmative answer.

\section{The Representation of the Minimal Tensor Product}

Suppose $V$ and $W$ are vector spaces with $\operatorname{dim}(V)<\infty$, then it is well known that there is a bijective correspondence between $V \otimes W \cong L\left(V^{*}, W\right)$ where $L\left(V^{*}, W\right)$ is the vector space of linear maps from $V^{*}$ into $W$. The bijective linear map is given by

$$
\Sigma v_{i} \otimes w_{i} \mapsto \widehat{\Sigma v_{i} \otimes} w_{i} \quad \text { where } \quad \widehat{\Sigma v_{i} \otimes w_{i}}(f)=\Sigma f\left(v_{i}\right) w_{i} .
$$

This identification plays an important role in the characterization of minimal tensor products both in Banach space and operator space theory (see 4] e.g.) . (Note that every linear map defined from a finite dimensional operator space is completely bounded which can be seen in 34.) The following is the operator system variant of this well known correspondence. In this section we will study various application of this equivalence. The first part is [22, Lem. 8.4].

Proposition 6.1. Let $\mathcal{S}$ and $\mathcal{T}$ be operator systems where $\operatorname{dim}(\mathcal{S})$ is finite. Then there is a bijective correspondence between

$$
\left(\mathcal{S} \otimes_{\min } \mathcal{T}\right)^{+} \longleftrightarrow C P\left(\mathcal{S}^{d}, \mathcal{T}\right)
$$


That is, a finite sum $\Sigma s_{i} \otimes t_{i}$ is positive if and only if the corresponding map $\widehat{\Sigma s_{i} \otimes t_{i}}$ is completely positive from $\mathcal{S}^{d}$ into $\mathcal{T}$. In particular every linear map from $\mathcal{S}^{\text {d }}$ into $\mathcal{T}$ can be written as a linear combination of completely positive maps.

Proof. The bijective correspondence is already shown in [22]. Now let $\mathcal{S}=\operatorname{span}\{e=$ $\left.s_{1}, s_{2}, \ldots, s_{n}\right\}$ written in the special basis form as in Lemma 2.5 and let $\mathcal{S}^{d}=\operatorname{span}\left\{\delta_{1}, \delta_{2}, \ldots, \delta_{n}\right\}$ written as the corresponding dual basis form. Consider a linear map $\varphi: \mathcal{S}^{d} \rightarrow \mathcal{T}$ where $\varphi\left(\delta_{i}\right)=t_{i}$. Now $\Sigma\left(s_{i} \otimes t_{i}\right)$ can be written as linear combination of positives in $\mathcal{S} \otimes_{\min } \mathcal{T}$, say $\Sigma\left(s_{i} \otimes t_{i}\right)=x_{1}-x_{2}+i x_{3}-i x_{4}$ where each $x_{i}$ is positive. By the first part, the corresponding maps $\hat{x_{i}}$ are completely positive from $\mathcal{S}^{d}$ into $\mathcal{T}$ and clearly $\varphi=\hat{x_{1}}-\hat{x_{2}}+i \hat{x_{3}}-i \hat{x_{4}}$. This finishes the proof.

Corollary 6.2. If $\mathcal{S}$ and $\mathcal{T}$ are operator systems with $\operatorname{dim}(\mathcal{S})<\infty$ then every linear map from $\mathcal{S}$ to $\mathcal{T}$ can be written as a linear combination of completely positive maps.

Aside: Supposing $\mathcal{S}$ and $\mathcal{T}$ are operator systems with $\operatorname{dim}(\mathcal{S})<\infty$ then $C B(\mathcal{S}, \mathcal{T})$ has a structure of an operator system: The involution is given by $\varphi^{*}(s)=\varphi\left(s^{*}\right)^{*}$ and the positive cones structures can be describe as

$$
\left(\varphi_{i j}\right) \in M_{n}(C B(\mathcal{S}, \mathcal{T})) \text { is positive if the map } \mathcal{S} \ni s \mapsto\left(\varphi_{i j}(s)\right) \in M_{n}(\mathcal{T}) \text { is cp. }
$$

The non-canonical Archimedean order unit can be chosen to be $\tilde{\delta}=\delta(\cdot) e_{\mathcal{T}}$ where $\delta$ is a faithful state on $\mathcal{S}$. Moreover we obtain the following identity

$$
\mathcal{S}^{d} \otimes_{\min } \mathcal{T}=C B(\mathcal{S}, \mathcal{T})
$$

unitally and completely order isomorphicaly. Of course, this also means that $\mathcal{S} \otimes_{\min } \mathcal{T}=$ $C B\left(\mathcal{S}^{d}, \mathcal{T}\right)$ where the identity of $C B\left(\mathcal{S}^{d}, \mathcal{T}\right)$ is chosen to be $\hat{e}(\cdot) e_{\mathcal{T}}$.

Proposition 6.1 has several important consequences. We want to start with the following duality property between the minimal and the maximal tensor products given in [10]. We also include the proof as it relies on the representation of the tensor products.

Theorem 6.3 (Farenick, Paulsen). For finite dimensional operator systems $\mathcal{S}$ and $\mathcal{T}$ we have the following unital complete order isomorphisms:

$$
\left(\mathcal{S} \otimes_{\max } \mathcal{T}\right)^{d}=\mathcal{S}^{d} \otimes_{\min } \mathcal{T}^{d} \quad \text { and } \quad\left(\mathcal{S} \otimes_{\min } \mathcal{T}\right)^{d}=\mathcal{S}^{d} \otimes_{\max } \mathcal{T}^{d} .
$$

More precisely, if $\delta_{\mathcal{S}}$ and $\delta_{\mathcal{T}}$ are faithful states on $\mathcal{S}$ and $\mathcal{T}$, resp., which we set as Archimedean order units, then $\delta_{\mathcal{S}} \otimes \delta_{\mathcal{T}}$ is again a faithful state on $\mathcal{S} \otimes_{\min } \mathcal{T}$ and $\mathcal{S} \otimes_{\max } \mathcal{T}$ when considered as a linear functional.

Proof. We first show that $\mathcal{S} \otimes_{\min } \mathcal{T}$ and $\left(\mathcal{S}^{d} \otimes_{\max } \mathcal{T}^{d}\right)^{d}$ are completely order isomorphic. Note that

$$
\left(\mathcal{S} \otimes_{\min } \mathcal{T}\right)^{+}=C P\left(\mathcal{S}^{d}, \mathcal{T}\right)=\left(\mathcal{S}^{d} \otimes_{\max } \mathcal{T}^{d}\right)^{d,+}
$$

Here the second equation follows from the representation of the maximal tensor product that we discussed in Subsection 3.2. Therefore, we obtain that a positive linear functional on $\mathcal{S}^{d} \otimes_{\max } \mathcal{T}^{d}$ corresponds to a positive element in $\mathcal{S} \otimes_{\min } \mathcal{T}$. This shows that the bijective linear map

$$
\mathcal{S} \otimes_{\min } \mathcal{T} \rightarrow\left(\mathcal{S}^{d} \otimes_{\text {max }} \mathcal{T}^{d}\right)^{d} \quad s \otimes t \mapsto s \dot{\otimes} t \text { where } s \dot{\otimes} t\left(\Sigma f_{i} \otimes g_{i}\right)=\Sigma f_{i}(s) g_{i}(t)
$$

is an order isomorphism. To see that it is an complete order isomorphism we can reduce the matricial levels to a ground level as follows. First note that

$$
M_{n}(\mathcal{S}) \otimes_{\min } \mathcal{T} \text { and }\left(M_{n}(\mathcal{S})^{d} \otimes_{\max } \mathcal{T}^{d}\right)^{d}
$$


are order isomorphic. The left hand side can be identified with $M_{n}\left(\mathcal{S} \otimes_{\min } \mathcal{T}\right)$. On the other hand, for any operator system $\mathcal{R}$ we have the identification $M_{n}\left(\mathcal{R}^{d}\right)=\left(M_{n}(\mathcal{R})\right)^{d}$ given by $\left(f_{i j}\right) \mapsto F$ where $F\left(r_{i j}\right)=\Sigma f_{i j}\left(r_{i j}\right)$. In fact, we first identify $M_{n}\left(\mathcal{R}^{d}\right)$ with linear operators from $\mathcal{R}$ into $M_{n}$ (where we use the definition of positivity) and these linear operators are identified with linear functionals on $M_{n}(\mathcal{R})$ (see [33, Thm. 6.1.], e.g.). By the associativity of the maximal tensor product we have that the right hand side can be identified with

$$
\left(M_{n}\left(\mathcal{S}^{d}\right) \otimes_{\max } \mathcal{T}^{d}\right)^{d}=\left(M_{n}\left(\mathcal{S}^{d} \otimes_{\max } \mathcal{T}^{d}\right)\right)^{d}=M_{n}\left(\left(\mathcal{S}^{d} \otimes_{\max } \mathcal{T}^{d}\right)^{d}\right)
$$

Thus the above map is completely order isomorphic. We may suppose that these operator systems have the same unit by simply declaring $e_{\mathcal{S}} \dot{\otimes} e_{\mathcal{T}}$ as the Archimedean order unit on $\left(\mathcal{S}^{d} \otimes_{\max } \mathcal{T}^{d}\right)^{d}$. (Since both of these matrix ordered spaces are completely order isomorphic, clearly, $e_{\mathcal{S}} \dot{\otimes} e_{\mathcal{T}}$ plays the same role on $\left(\mathcal{S}^{d} \otimes_{\max } \mathcal{T}^{d}\right)^{d}$.) Finally by taking appropriate duals, we obtain both first and second desired identifications.

This duality correspondence allows us to recover the following special case about the projectivity of the maximal tensor product given in [16].

Theorem 6.4. Let $\mathcal{S}$ and $\mathcal{T}$ be finite dimensional operator systems and $J \subset \mathcal{S}$ be a null subspace. Then $J \otimes \mathcal{T} \subset \mathcal{S} \otimes_{\max } \mathcal{T}$ is a null subspace and we have that

$$
\left(\mathcal{S} \otimes_{\max } \mathcal{T}\right) /(J \otimes \mathcal{T})=(\mathcal{S} / J) \otimes_{\max } \mathcal{T}
$$

In other words, the induced map $\mathcal{S} \otimes_{\max } \mathcal{T} \longrightarrow(\mathcal{S} / J) \otimes_{\max } \mathcal{T}$ is a unital quotient map.

Proof. Proposition 2.7 ensures that $(\mathcal{S} / J)^{d}$ is an operator subsystem of $\mathcal{S}^{d}$. Thus, by using the injectivity of the minimal tensor product, we have that

$$
(\mathcal{S} / J)^{d} \otimes_{\min } \mathcal{T}^{d} \subset \mathcal{S}^{d} \otimes_{\min } \mathcal{T}^{d}
$$

Now, Theorem 2.8 (and the remark thereafter) ensure that the adjoint of this map is a quotient map whose kernel is a null subspace. Thus, by using the above result, the adjoint of this inclusion, i.e., the natural map below

$$
\mathcal{S} \otimes_{\max } \mathcal{T} \longrightarrow(\mathcal{S} / J) \otimes_{\max } \mathcal{T}
$$

is a quotient map. By a dimension count argument its kernel is $J \otimes \mathcal{T}$ which is a null subspace.

With the following lemma we resolve some technical issues. Its proof is again based on the representation of the minimal tensor product.

Lemma 6.5. Let $\mathcal{S}$ be a finite dimensional operator system, $\mathcal{A}$ be a $C^{*}$-algebra and $I$ be an ideal in $\mathcal{A}$. Then the following are equivalent:

(1) For all $n$, every ucp map $\varphi: \mathcal{S} \rightarrow M_{n}(\mathcal{A}) / M_{n}(I)$ has cp lift on $M_{n}(\mathcal{A})$.

(2) For all $n$, every cp map $\varphi: \mathcal{S} \rightarrow M_{n}(\mathcal{A}) / M_{n}(I)$ has cp lift on $M_{n}(\mathcal{A})$.

(3) We have the unital complete order isomorphism

$$
\left(\mathcal{S}^{d} \otimes_{\min } \mathcal{A}\right) /\left(\mathcal{S}^{d} \otimes I\right)=\mathcal{S}^{d} \otimes_{\min }(\mathcal{A} / I) .
$$

Proof. We first remark that if we replace "for all $n$ " with "for $n=1$ " in (1) and (2) and remove "complete" in (3) and prove the lemma this way then the the original arguments automatically satisfied. In fact this follows from the identifications

$M_{n}\left(\left(\mathcal{S}^{d} \otimes_{\min } \mathcal{A}\right) /\left(\mathcal{S}^{d} \otimes I\right)\right)=M_{n}\left(\mathcal{S}^{d} \otimes_{\min } \mathcal{A}\right) / M_{n}\left(\mathcal{S}^{d} \otimes I\right)=\left(\mathcal{S}^{d} \otimes_{\min } M_{n}(\mathcal{A})\right) /\left(\mathcal{S}^{d} \otimes M_{n}(I)\right)$ and $M_{n}\left(\mathcal{S}^{d} \otimes_{\min }(\mathcal{A} / I)\right)=\mathcal{S}^{d} \otimes_{\min } M_{n}(\mathcal{A} / I)$. So we will prove the equivalences only for the ground level. Clearly (2) implies (1). (3) implies (2) is also easy. The cp map $\varphi: \mathcal{S} \rightarrow \mathcal{A} / I$ 
corresponds to a positive element in $\mathcal{S}^{d} \otimes_{\min }(\mathcal{A} / I)$. Since we assumed (3) and the first quotient in (3) is proximinal (see Cor. 5.15 of [21] e.g.) it follows that $u$ is quotient of a positive element $v$ in $\mathcal{S}^{d} \otimes_{\min } \mathcal{A}$. Now, again by using the representation of minimal tensor product, $v$ corresponds a cp map $\tilde{\varphi}: \mathcal{S} \rightarrow \mathcal{A}$. It is not hard to show that $\tilde{\varphi}$ is a lift of $\varphi$.

We finally show that (1) implies (3). First note that (1) implies the following: Whenever $\phi: \mathcal{S} \rightarrow \mathcal{A} / I$ is a cp map with $\phi(e)$ is invertible then $\phi$ has cp lift on $\mathcal{A}$. In fact if we set $\psi=\phi(e)^{-1 / 2} \phi(\cdot) \phi(e)^{-1 / 2}$ then $\psi$ is a ucp map and hence has a cp lift $\tilde{\psi}$ on $\mathcal{A}$. Now, if $a$ is in $\mathcal{A}^{+}$with $a+I=\phi(e)^{1 / 2}$ then it is easy to see that the cp map $a \tilde{\psi}(\cdot) a$ is a lift of $\phi$. Secondly, we remark that the induced map from $\left(\mathcal{S}^{d} \otimes_{\min } \mathcal{A}\right) /\left(\mathcal{S}^{d} \otimes I\right)$ to $\mathcal{S}^{d} \otimes_{\min }(\mathcal{A} / I)$ is already bijective and ucp (see Remark 4.8). Thus we need to show that its inverse is positive. So let $u$ be positive in $\mathcal{S}^{d} \otimes_{\min }(\mathcal{A} / I)$ and set $u_{\epsilon}=u+\epsilon 1$ for $\epsilon>0$, where 1 is the unit of $\mathcal{S}^{d} \otimes_{\min }(\mathcal{A} / I)$. (Note: $1=f \otimes \dot{e}_{\mathcal{A}}$ where $f$ is a faithful sate on $\mathcal{S}$.) Since $u$ and $u_{\epsilon}$ are positive elements they corresponds to cp maps $\varphi$ and $\varphi_{\epsilon}$ from $\mathcal{S}$ into $\mathcal{A} / I$, respectively. It is not hard to see that $\varphi_{\epsilon}\left(e_{\mathcal{S}}\right)=\varphi\left(e_{\mathcal{S}}\right)+\epsilon \dot{e}_{\mathcal{A}}$. This means that $\varphi_{\epsilon}\left(e_{\mathcal{S}}\right)$ is invertible and so it has a cp lift $\tilde{\varphi}_{\epsilon}$ from $\mathcal{S}$ into $\mathcal{A}$. This again corresponds to a positive element $U_{\epsilon}$ in $\mathcal{S}^{d} \otimes_{\min } \mathcal{A}$. Now it is not hard to see that the positive element $U_{\epsilon}+\mathcal{S}^{d} \otimes I$ is the inverse image of $u_{\epsilon}=u+\epsilon 1$ for every $\epsilon>0$. This is enough to conclude that two operator systems in (3) are order isomorphic.

Theorem 6.6. Let $\mathcal{S}$ be a finite dimensional operator system. Then $\mathcal{S}$ has the lifting property if and only if $\mathcal{S}^{d}$ is exact (and vice versa). In other words, $\mathcal{S}$ is (min,er)-nuclear if and only if $\mathcal{S}^{d}$ is (min,el)-nuclear.

Proof. The proof is based on Lemma 6.5. If $\mathcal{S}$ has the lifting property then (1) in the same lemma will be satisfied for every $\mathrm{C}^{*}$-algebra and ideal. Thus (3) implies that $\mathcal{S}^{d}$ is exact. The reverse direction similar. Since $\mathcal{S}^{d d}=\mathcal{S}$ we clearly have that $\mathcal{S}$ is exact if and only if $\mathcal{S}^{d}$ has the lifting property.

Theorem 6.7. If the Kirchberg conjecture has an affirmative answer then, in the finite dimensional case, $C^{*}$-nuclearity is preserved under duality, that is, if $\mathcal{S}$ is $C^{*}$-nuclear then $\mathcal{S}^{d}$ is again $C^{*}$-nuclear.

Proof. Let $\mathcal{S}$ be a finite dimensional $\mathrm{C}^{*}$-nuclear operator system. In particular $\mathcal{S}$ is exact and has the lifting property. By the above result $\mathcal{S}^{d}$ has both of these properties. Now if the Kirchberg conjecture is true then Theorem 5.10 implies that $\mathcal{S}^{d}$ has DCEP. It is easy to see that exactness and DCEP together imply $\mathrm{C}^{*}$-nuclearity. Thus, $\mathcal{S}^{d}$ is $\mathrm{C}^{*}$-nuclear.

The local lifting property of a $\mathrm{C}^{*}$-algebra, in general, does not pass to its quotients by ideals. In fact it is well known that every $\mathrm{C}^{*}$-algebra is the quotient of a full $\mathrm{C}^{*}$-algebra of a free group which has the local lifting property however there are $\mathrm{C}^{*}$-algebras without this property. On the finite dimensional operator systems this situation is different:

Theorem 6.8. Let $\mathcal{S}$ be a finite dimensional operator system and let $J$ be a null subspace of $\mathcal{S}$. If $\mathcal{S}$ has the lifting property then $\mathcal{S} / J$ has the same property.

Proof. Recall from Proposition 2.7 that $(\mathcal{S} / J)^{d}$ is an operator subsystem of $\mathcal{S}^{d}$. Since $\mathcal{S}$ has the lifting property then $\mathcal{S}^{d}$ is exact by Theorem 6.6. Proposition 4.10 states that exactness passes to operator subsystems so $(\mathcal{S} / J)^{d}$ is exact and consequently using Theorem 6.6 again it follows that $\mathcal{S} / J$ has the lifting property.

Example 6.9. We define $J_{n} \subset M_{n}$ as the subspace which includes all the diagonal operators with 0 trace. Clearly $J_{n}$ is a null subspace and consequently, by Proposition 2.4, it is a kernel. Since $M_{n}$ is a nuclear $\mathrm{C}^{*}$-algebra, it is a (min,max)-nuclear operator system. In particular, it is (min,er)-nuclear equivalently has the lifting property. Thus, by the above theorem $M_{n} / J_{n}$ has the lifting property. We will come back to this example in later sections. 
The lifting property is also stable when passing to universal $\mathrm{C}^{*}$-algebras. The following result is an unpublished work of Ivan Todorov which he informed me of during this research. The operator space analogue can be seen in [32].

Theorem 6.10. Let $\mathcal{S}$ be a finite dimensional operator system. Then $\mathcal{S}$ has lifting property if and only if $C_{u}^{*}(\mathcal{S})$ has $L L P$.

Proof. First suppose that $\mathcal{S}$ has the lifting property. Let $\pi: C_{u}^{*}(\mathcal{S}) \rightarrow \mathcal{A} / I$ be a unital *homomorphism. (Note: As pointed out in [41, Rem. 16.3 (ii)] it is enough to consider the the unital representations to verify the LLP of a $\mathrm{C}^{*}$-algebra.) Let $\pi_{0}$ be the restriction of $\pi$ on $\mathcal{S}$. By using the local lifting property of $\mathcal{S}$ we have a ucp map $\varphi$ from $\mathcal{S}$ to $\mathcal{A}$ which lifts $\pi_{0}$. Let $\rho: C_{u}^{*}(\mathcal{S}) \rightarrow \mathcal{A}$ be the unital $*$-homomorphism extending $\varphi$. It is elementary to show that $\rho$ is a lift of $\pi$. Conversely suppose that $C_{u}^{*}(\mathcal{S})$ has LLP. Let $\varphi: \mathcal{S} \rightarrow \mathcal{A} / I$ be a ucp map. Let $\pi: C_{u}^{*}(\mathcal{S}) \rightarrow \mathcal{A} / I$ be the associated $*$-homomorphism. Now since $\mathcal{S}$ is a finite dimensional operator subsystem of $C_{u}^{*}(\mathcal{S})$, the restriction of $\pi$ on $\mathcal{S}$, namely $\varphi$, lifts to a ucp map on $\mathcal{A}$. This completes the proof.

For some other applications the following result will be useful.

Proposition 6.11. Suppose $\mathcal{S}$ and $\mathcal{T}$ are two finite dimensional operator systems with the same dimensions. Then there is a surjective ucp map $\varphi: \mathcal{S} \rightarrow \mathcal{T}$.

Proof. Let $\mathcal{S}=\operatorname{span}\left\{e=s_{1}, s_{2}, \ldots, s_{n}\right\}$ and $\mathcal{T}=\operatorname{span}\left\{e=t_{1}, t_{2}, \ldots, t_{n}\right\}$ written in the special basis form as in Lemma 2.5. Let $\mathcal{S}^{d}=\operatorname{span}\left\{\delta_{1}, \delta_{2}, \ldots, \delta_{n}\right\}$ given in the corresponding dual basis form. Recall that $\delta_{1}$ is an Archimedean order unit for $\mathcal{S}^{d}$. Note that

$$
\delta_{2} \otimes t_{2}+\cdots \delta_{n} \otimes t_{n}
$$

is a self-adjoint element of $\mathcal{S}^{d} \otimes_{\min } \mathcal{T}$ and consequently there is a large $M$ such that

$$
\delta_{1} \otimes e+\left(\delta_{2} \otimes t_{2}+\cdots \delta_{n} \otimes t_{n}\right) / M
$$

is positive. Now by using Proposition 6.1 it is elementary to see that the corresponding completely positive map from $\mathcal{S}$ to $\mathcal{T}$ is unital and surjective.

Corollary 6.12. Suppose $\mathcal{S}$ and $\mathcal{T}$ are operator systems with $\operatorname{dim}(\mathcal{T})$ finite and $\operatorname{dim}(\mathcal{T}) \leq$ $\operatorname{dim}(\mathcal{S})$. Then there is a surjective ucp map from $\mathcal{S}$ to $\mathcal{T}$.

Proof. Suppose $\operatorname{dim}(\mathcal{T})=n$ and let $\mathcal{S}_{0}$ be an $n$-dimensional operator subsystem of $\mathcal{S}$. By using the above proposition there is a surjective ucp map from $\mathcal{S}_{0}$ onto $\mathbb{C}^{n}$. Since $\mathbb{C}^{n}$ is injective this map extends to a ucp map from $\mathcal{S}$ on $\mathbb{C}^{n}$. Now again by using the above proposition we have surjective ucp map from $\mathbb{C}^{n}$ onto $\mathcal{T}$. Composition of these two maps is surjective and ucp.

In [27] Kirchberg and Wasserman exemplify the behavior of universal $\mathrm{C}^{*}$-algebras of some low dimensional operator systems. More precisely they show that:

(1) $C_{u}^{*}\left(\mathbb{C}^{2}\right)$ is unitally $*$-isomorphic to $C[0,1]$, in particular, it is nuclear.

(2) $C_{u}^{*}\left(\mathbb{C}^{3}\right)$ is not exact.

By using Corollary 6.12 we obtain the following:

\section{Proposition 6.13.}

(1) If $\mathcal{S}$ is a two dimensional operator system then $C_{u}^{*}(\mathcal{S})$ is nuclear. In particular $\mathcal{S}$ is (min,c)-nuclear (equivalently $C^{*}$-nuclear).

(2) If $\mathcal{S}$ is an operator system with $\operatorname{dim}(\mathcal{S}) \geq 3$ then $C_{u}^{*}(\mathcal{S})$ is not exact. 
Proof. Both parts of the proof are based on Corollary 6.12, Suppose $\mathcal{S}$ is a two dimensional operator system. Let $\varphi: \mathbb{C}^{2} \rightarrow \mathcal{S}$ be a surjective ucp map and let $\pi: C_{u}^{*}\left(\mathbb{C}^{2}\right) \rightarrow C_{u}^{*}(\mathcal{S})$ be the corresponding unital $*$-homomorphism. Note that $\pi$ is surjective so $C_{u}^{*}\left(\mathbb{C}^{2}\right) / k e r(\pi)$ and $C_{u}^{*}(\mathcal{S})$ are $*$-isomorphic $\mathrm{C}^{*}$-algebras. This means that $C_{u}^{*}(\mathcal{S})$ is quotient of a nuclear $\mathrm{C}^{*}$-algebra and consequently it is nuclear (see [8] e.g.). To see that $\mathcal{S}$ is (min,c)-nuclear first fix an operator system $\mathcal{T}$. We have the inclusions

$$
\mathcal{S} \otimes_{\min } \mathcal{T} \subset C_{u}^{*}(\mathcal{S}) \otimes_{\min } \mathcal{T} \text { and } \mathcal{S} \otimes_{c} \mathcal{T} \subset C_{u}^{*}(\mathcal{S}) \otimes_{\max } \mathcal{T}
$$

Since the tensor products on the right coincide it follows that $\mathcal{S}$ is (min,c)-nuclear.

Now let $\mathcal{S}$ be an operator system with $\operatorname{dim}(\mathcal{S}) \geq 3$. Assume for a contradiction that $C_{u}^{*}(\mathcal{S})$ is exact. Let $\varphi: \mathcal{S} \rightarrow \mathbb{C}^{3}$ be a surjective ucp map and let $\pi: C_{u}^{*}(\mathcal{S}) \rightarrow C_{u}^{*}\left(\mathbb{C}^{3}\right)$ be the corresponding unital $*$-homomorphism which is surjective. This means that $C_{u}^{*}\left(\mathbb{C}^{3}\right)$ is a quotient of an exact $\mathrm{C}^{*}$-algebra. So another result of Kirchberg [24], which states that exactness passes to quotients by ideals, requires $C_{u}^{*}\left(\mathbb{C}^{3}\right)$ to be exact which is a contradiction.

For another application of Corollary 6.12 we need some preliminary results. If $X$ is an operator space then there is an, essentially unique, operator system $\mathcal{T}_{X}$ together with a completely isometric inclusion $i: X \hookrightarrow \mathcal{T}_{X}$ such that it satisfies the following universal property: For every completely contractive map $\phi: X \rightarrow \mathcal{S}$, where $\mathcal{S}$ is an operator system, there exists a unique ucp map $\varphi: \mathcal{T}_{X} \rightarrow \mathcal{S}$ such that $\varphi(i(x))=\phi(x)$ for every $x$ in $X$.

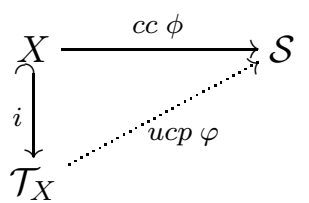

To see the existence of $\mathcal{T}_{X}$ one can first consider the universal unital $\mathrm{C}^{*}$-algebra $C_{u}^{*}\langle X\rangle$ of the operator space $X$. Recall that it has the following universal property: Every completely contractive map defined from $X$ into a unital $\mathrm{C}^{*}$-algebra $\mathcal{A}$ extends uniquely to a unital *-homomorphism. (See [41, Thm. 8.14] e.g.) Now let the span of $X, X^{*}$ and the unit $e$ be $\mathcal{T}_{X}$. (Also note that the image can be taken to an operator system.) If $X_{0}$ is an operator subspace of $X$ then we have a unital complete order embedding $\mathcal{T}_{X_{0}} \subset \mathcal{T}_{X}$. We leave the proof of this as an exercise. Also, the following identification is immediate:

$$
C_{u}^{*}\langle X\rangle=C_{u}^{*}\left(\mathcal{T}_{X}\right)
$$

Recall that an operator space $X$ is said to have the $\lambda$-operator space local lifting property $(\lambda$ OLLP) if the following holds for every unital $\mathrm{C}^{*}$-algebra $\mathcal{A}$ and ideal $I$ in $\mathcal{A}$. If $\phi: X \rightarrow \mathcal{A} / I$ is a completely contractive (cc) map and $X_{0}$ is a finite dimensional operator subspace of $X$ then $\left.\phi\right|_{X_{0}}$ has a lift $\tilde{\phi}_{0}$ on $\mathcal{A}$ with $\left\|\tilde{\phi}_{0}\right\|_{c b} \leq \lambda$. We claim that:

Proposition 6.14. Let $X$ be an operator space. Then $X$ has 1-OLLP if and only if $\mathcal{T}_{X}$ has osLLP.

Proof. Let $\mathcal{A}$ be a unital $\mathrm{C}^{*}$-algebra and $I$ be an ideal in $\mathcal{A}$. First suppose that $X$ has 1 OLLP. Let $\varphi: \mathcal{T}_{X} \rightarrow \mathcal{A} / I$ be a ucp map and let $\mathcal{T}_{0}$ be a finite dimensional operator subsystem of $\mathcal{T}_{X}$. Clearly we can find a finite dimensional subspace $X_{0}$ of $X$ such that the operator system generated by $X_{0}$, which is actually $\mathcal{T}_{X_{0}}$, contains $\mathcal{T}_{0}$. Note that $\left.\varphi\right|_{X}$ is cc and so its restriction on $X_{0}$ has a cc lift on $\mathcal{A}$. Now by using the universal property of $\mathcal{T}_{X_{0}}$ we obtain a ucp map from $\mathcal{T}_{X_{0}}$ on $\mathcal{A}$. Now the restriction of this map on $\mathcal{T}_{0}$ is a ucp lift on $\mathcal{A}$.

Conversely suppose $\mathcal{T}_{X}$ has osLLP and let $\phi: X \rightarrow \mathcal{A} / I$ be a cc map. This map has a ucp extension $\varphi$ on $\mathcal{T}_{X}$. Let $X_{0}$ be a finite dimensional operator subspace of $X$. Clearly $\mathcal{T}_{X_{0}}$ is a 
finite dimensional operator subsystem of $\mathcal{T}_{X}$ and consequently $\varphi$, when restricted to $\mathcal{T}_{X_{0}}$ has a ucp lift on $\mathcal{A}$. Finally restriction of this lift on $X_{0}$ is cc. This finishes the proof.

When $X=\mathbb{C}, \mathcal{T}_{X}$ is a three dimensional operator system. The following is from [25].

Proposition 6.15. The following are equivalent:

(1) The Kirchberg conjecture has an affirmative answer.

(2) $C_{u}^{*}\langle\mathbb{C}\rangle$ has WEP.

Depending heavily on this characterization we can obtain further equivalences. (The equivalence of (1) and (4) was pointed out by Vern Paulsen.)

Proposition 6.16. The following are equivalent:

(1) The Kirchberg conjecture has an affirmative answer.

(2) There exists a three dimensional operator system $\mathcal{S}$ such that $C_{u}^{*}(\mathcal{S})$ has WEP.

(3) There exists an operator system $\mathcal{S}$ with $\operatorname{dim}(\mathcal{S}) \geq 3$ such that $C_{u}^{*}(\mathcal{S})$ has WEP.

(4) $C_{u}^{*}\left(M_{2}\right)$ has WEP.

Proof. Clearly (4) implies (3). To see that (3) implies (2), let $\mathcal{S}$ be an operator system with $\operatorname{dim}(\mathcal{S}) \geq 3$ such that $C_{u}^{*}(\mathcal{S})$ has WEP. Let $\mathcal{T}$ be a three dimensional operator system with the lifting property. (For example $\mathbb{C}^{3}$ ). By using Corollary 6.12, we know that there is surjective ucp map $\varphi$ from $\mathcal{S}$ to $\mathcal{T}$. Note that this ucp map extends to surjective *homomorphism $\pi: C_{u}^{*}(\mathcal{S}) \rightarrow C_{u}^{*}(\mathcal{T})$. Since $C_{u}^{*}(\mathcal{S}) / \operatorname{ker}(\pi)$ and $C_{u}^{*}(\mathcal{T})$ are ${ }^{*}$-isomorphic $\mathrm{C}^{*}$ algebras we obtain that $C_{u}^{*}(\mathcal{T})$ is QWEP. Also by Theorem 6.10, $C_{u}^{*}(\mathcal{T})$ has LLP. A well known result of Kirchberg states that QWEP and LLP together imply WEP ([25]). Thus, (3) implies (2). Now we will show (2) implies (1). By using the above result of Kirchberg it is enough to prove that $C_{u}^{*}\langle\mathbb{C}\rangle$ has WEP. Recall that $C_{u}^{*}\langle\mathbb{C}\rangle=C_{u}^{*}\left(\mathcal{T}_{\mathbb{C}}\right)$. Since $\mathbb{C}$ has 1 OLLP it follows that $\mathcal{T}_{\mathbb{C}}$ has the lifting property. By Theorem 6.10 $C_{u}^{*}\left(\mathcal{T}_{\mathbb{C}}\right)$ has LLP. By using an argument that we used in the implication $(3) \Rightarrow(2)$ it is easy to see that existence of a three dimensional operator system with WEP implies $C_{u}^{*}\left(\mathcal{T}_{\mathbb{C}}\right)$ is QWEP. Consequently $C_{u}^{*}\left(\mathcal{T}_{\mathbb{C}}\right)=C_{u}^{*}\langle\mathbb{C}\rangle$ has WEP. Finally to see that (1) implies (4), note that $C_{u}^{*}\left(M_{2}\right)$ has LLP (since $M_{2}$ has the lifting property). So assuming KC it follows that $C_{u}^{*}\left(M_{2}\right)$ has WEP.

\section{Further Exactness and Lifting Properties}

We first want to review some instances where the operator space and the operator system quotients are completely isometric. Then by using a result of Ozawa [31, we obtain simpler exactness and local liftability conditions for operator systems. This follows the track of Pisier and Ozawa's approach for operator spaces (see Thm. 16.10 and Rem. 17.6 of [41], e.g.). Let $\mathcal{S}$ be operator system $\mathcal{A}$ be a unital $\mathrm{C}^{*}$-algebra and $I$ be an ideal in $\mathcal{A}$. As we pointed out in Subsection 4.5, $\mathcal{S} \bar{\otimes} I \subset \mathcal{S} \hat{\otimes}_{\min } \mathcal{A}$ is a kernel. $\left(\hat{\otimes}_{\text {min }}\right.$ denotes the completed minimal tensor product and $\bar{\otimes}$ is the closure of the algebraic tensor product in the larger space.) Moreover, the canonical operator spaces structure on the operator system quotient

$$
\left(\mathcal{S} \hat{\otimes}_{\min } \mathcal{A}\right) /(\mathcal{S} \bar{\otimes} I)
$$

coincides with the operator space quotient of $\mathcal{S} \hat{\otimes}_{\text {min }} \mathcal{A}$ by its closed subspace $\mathcal{S} \bar{\otimes} I$. (See [21, Thm. 5.1]). Also recall from Remark 4.8 that when $\mathcal{S}$ is finite dimensional then the minimal tensor of $\mathcal{S}$ with a $\mathrm{C}^{*}$-algebra is already a completed object so we will use $\otimes_{\min }$ instead of $\hat{\otimes}_{\text {min }}$. Similarly if $I$ is an ideal in a $\mathrm{C}^{*}$-algebra $\mathcal{A}$ then $\mathcal{S} \bar{\otimes} \mathcal{I}$ coincides with the algebraic tensor product $\mathcal{S} \otimes I$. So we omit the bar over the tensor product. 
Notation: For simplicity in the following results we let $\mathbb{B}$ denote $B\left(l^{2}\right)$ and $\mathbb{K}$ stands for the ideal of compact operators in $B\left(l^{2}\right)$.

Suppose $\mathcal{A}$ is a unital $\mathrm{C}^{*}$-algebra and $I$ is an ideal in $\mathcal{A}$. Let

$$
C=\{\phi: \mathcal{A} \rightarrow \mathbb{B}: \phi \text { is ucp and } \phi(I) \subseteq \mathbb{K}\} .
$$

For $\phi$ in $C$ we use the notation $\dot{\phi}$ for the induced map $\mathcal{A} / I \rightarrow \mathbb{B} / \mathbb{K}$. If $X$ is a finite dimensional operator space then $\bar{\phi}$ denotes the corresponding map

$$
(X \hat{\otimes} \mathcal{A}) /(X \otimes I) \rightarrow(X \hat{\otimes} \mathbb{B}) /(X \otimes \mathbb{K}) .
$$

where $\hat{\otimes}$ is the minimal operator space tensor product. We are ready to state:

Proposition 7.1 (Ozawa, [31]). , Let $X$ be a finite dimensional operator space. If $\mathcal{A}$ is a unital separable $C^{*}$-algebra and $\mathcal{I}$ is an ideal in $\mathcal{A}$ then for any $u$ in $X \otimes \mathcal{A} / I$ we have

$$
\|u\|_{X \hat{\otimes} \mathcal{A} / I}=\sup _{\phi \in C}\|(i d \otimes \dot{\phi})(u)\|_{X \hat{\otimes} \mathbb{B} / \mathbb{K}}
$$

and for any $v$ in $(X \hat{\otimes} \mathcal{A}) /(X \otimes I)$

$$
\|v\|_{(X \hat{\otimes} \mathcal{A}) /(X \otimes I)}=\sup _{\phi \in C}\|(i d \otimes \bar{\phi})(v)\|_{(X \hat{\otimes} \mathbb{B}) /(X \otimes \mathbb{K})} .
$$

Before stating the following result we want to emphasize that the the minimal operator system and the minimal operator space tensor products coincide. (In fact they are both spatial.) The exactness criteria in the next theorem is true for every operator system which we included as a corollary.

Theorem 7.2. Suppose $\mathcal{S}$ is a finite dimensional operator system. Then $\mathcal{S}$ is exact if and only if

$$
\left(\mathcal{S} \otimes_{\min } \mathbb{B}\right) /(\mathcal{S} \otimes \mathbb{K}) \cong \mathcal{S} \otimes_{\min } \mathbb{B} / \mathbb{K}
$$

Proof. One direction is clear. So suppose that $\left(\mathcal{S} \otimes_{\min } \mathbb{B}\right) /\left(\mathcal{S} \otimes_{\min } \mathbb{K}\right) \cong \mathcal{S} \otimes_{\min } \mathbb{B} / \mathbb{K}$. In particular this implies that the associated map is completely isometric. (Recall: The operator space quotient and operator system quotient has same operator space structure.) So using Ozawa's above result we have that for every separable unital $\mathrm{C}^{*}$-algebra $\mathcal{A}$ and ideal $I$ in $\mathcal{A}$ the associated map

$$
\left(\mathcal{S} \otimes_{\min } \mathcal{A}\right) /(\mathcal{S} \otimes I) \longrightarrow \mathcal{S} \otimes_{\min } \mathcal{A} / I
$$

is isometric. (Note: the minimal tensor product of operator systems coincides with the minimal operator space tensor product.) To see that it is complete isometry it is enough to consider the identification $M_{n}(\mathcal{A} / I)=M_{n}(\mathcal{A}) / M_{n}(I)$. Since a unital complete isometry is a complete order isomorphism we have that the exactness is satisfied for the separable case. Now suppose $\mathcal{A}$ is an arbitrary unital $\mathrm{C}^{*}$-algebra and $I$ is an ideal in $\mathcal{A}$. Assume for a contradiction that the associated map

$$
\left(\mathcal{S} \otimes_{\min } \mathcal{A}\right) /(\mathcal{S} \otimes I) \longrightarrow \mathcal{S} \otimes_{\min } \mathcal{A} / I
$$

is not a complete isometry. Again considering the identification $M_{n}(\mathcal{A} / I)=M_{n}(\mathcal{A}) / M_{n}(I)$ we may suppose that the map is not an isometry. This means that there is an element $u$ of $\mathcal{S} \otimes_{\text {min }} \mathcal{A}$ such that the norm of $u+\mathcal{S} \otimes I$ under this associated map is strictly smaller. Clearly $\mathcal{A}$ has a separable unital $\mathrm{C}^{*}$-subalgebra $\mathcal{A}_{0}$ such that $u$ belongs to $\mathcal{S} \otimes \mathcal{A}_{0}$. Let $I_{0}=\mathcal{A}_{0} \cap I$, which is an ideal in $\mathcal{A}_{0}$. Moreover we have $\mathcal{A}_{0} / I_{0} \subset \mathcal{A} / I$ so the injectivity of minimal tensor products ensures that

$$
\mathcal{S} \otimes_{\min } \mathcal{A}_{0} / I_{0} \subset \mathcal{S} \otimes_{\min } \mathcal{A} / I
$$


We also have the following sequence of ucp maps:

$$
\mathcal{S} \otimes_{\text {min }} \mathcal{A}_{0} \hookrightarrow \mathcal{S} \otimes_{\min } \mathcal{A} \rightarrow\left(\mathcal{S} \otimes_{\min } \mathcal{A}\right) /(\mathcal{S} \otimes I)
$$

which has the kernel $\mathcal{S} \otimes I_{0}$. So the associated map $\left(\mathcal{S} \otimes_{\min } \mathcal{A}_{0}\right) /\left(\mathcal{S} \otimes I_{0}\right) \rightarrow\left(\mathcal{S} \otimes_{\text {min }} \mathcal{A}\right) /(\mathcal{S} \otimes I)$ is ucp. Finally when we look at the following sequence of ucp maps

$$
\left(\mathcal{S} \otimes_{\min } \mathcal{A}_{0}\right) /\left(\mathcal{S} \otimes I_{0}\right) \rightarrow\left(\mathcal{S} \otimes_{\min } \mathcal{A}\right) /(\mathcal{S} \otimes I) \longrightarrow \mathcal{S} \otimes_{\min } \mathcal{A} / I \supset \mathcal{S} \otimes_{\min } \mathcal{A}_{0} / I_{0}
$$

the norm of the element $u+\mathcal{S} \otimes I_{0}$ is smaller. This is a contradiction as the exactness of $\mathcal{S}$ fails for a separable $\mathrm{C}^{*}$-algebra and ideal in it.

Corollary 7.3. Let $\mathcal{S}$ be an operator system. Then $\mathcal{S}$ is exact if and only if

$$
\left(\mathcal{S} \otimes_{\text {min }} \mathbb{B}\right) /(\mathcal{S} \bar{\otimes} \mathbb{K}) \cong \mathcal{S} \otimes_{\text {min }} \mathbb{B} / \mathbb{K} \text {. }
$$

Proof. One direction is trivial. So suppose exactness in $\mathbb{K} \subset \mathbb{B}$ satisfied. Let $\mathcal{S}_{0}$ be a finite dimensional operator subsystem. By using Corollary 5.6 of [21] we have that

$$
\frac{\mathcal{S}_{0} \otimes_{\min } \mathbb{B}}{\mathcal{S}_{0} \otimes \mathbb{K}} \subset \frac{\mathcal{S} \otimes_{\min } \mathbb{B}}{\mathcal{S} \bar{\otimes} \mathbb{K}}
$$

Similarly, by the injectivity of the minimal tensor product, we have $\mathcal{S}_{0} \otimes_{\text {min }} \mathbb{B} / \mathbb{K} \subset \mathcal{S} \otimes_{\text {min }}$ $\mathbb{B} / \mathbb{K}$. So for the operator system $\mathcal{S}_{0}$ the exactness condition for $\mathbb{K} \subset \mathbb{B}$ in Proposition 7.2 is satisfied and consequently it is exact. Since $\mathcal{S}_{0}$ is an arbitrary finite dimensional operator subsystem of $\mathcal{S}$, by Proposition 4.10, it follows that $\mathcal{S}$ is exact.

Recall from Theorem 6.6 that exactness and the lifting property are dual pairs. That is a finite dimensional operator system $\mathcal{S}$ has the lifting property if and only $\mathcal{S}^{d}$ is exact. This, together with Lemma 6.5, lead to the following simplification of the lifting property.

Proposition 7.4. A finite dimensional operator system $\mathcal{S}$ has the lifting property if and only if every ucp map defined from $\mathcal{S}$ into $\mathbb{B} / \mathbb{K}$ has a ucp lift on $\mathbb{B}$.

Proof. Recall from Section 4.2 that in the definition of osLLP the local cp lifts can be taken to be unital. This proves one direction. Conversely suppose that every ucp map defined from $\mathcal{S}$ into $\mathbb{B} / \mathbb{K}$ has a ucp lift on $\mathbb{B}$. This means that, for every $n$, every ucp map $\varphi: \mathcal{S} \rightarrow$ $M_{n}(\mathbb{B}) / M_{n}(\mathbb{K})$ has a ucp lift on $M_{n}(\mathbb{B})$. In fact this directly follows from the fact that $M_{n}(\mathbb{B})$ can be identified with $\mathbb{B}$ via a $\mathrm{C}^{*}$-algebraic isomorphism which preserves the compactness. Thus, $\mathcal{S}$ satisfies the property (1) in Lemma 6.5. So the dual system $\mathcal{S}^{d}$ has the property (3) in the same lemma. Now, Theorem 7.2 implies that $\mathcal{S}^{d}$ is exact. Finally, by Theorem 6.6, $\mathcal{S}$ has the lifting property.

\section{Coproducts of Operator Systems}

In this chapter we recall basic facts on the amalgamated direct sum of two operator systems over the unit introduced in [23] (or with the language of [13] coproduct of two operator systems) and we will show that it can be formed directly by using the operator system quotient theory. We show that the lifting property is preserved under coproducts. However the stability of the double commutant expectation property turns out to be related to Kirchberg Conjecture. Recall that if $\mathcal{S}$ and $\mathcal{T}$ are two operator systems then the coproduct $\mathcal{S} \oplus_{1} \mathcal{T}$ of $\mathcal{S}$ and $\mathcal{T}$ is an operator system together with unital complete order embeddings $i: \mathcal{S} \hookrightarrow \mathcal{S} \oplus_{1} \mathcal{T}$ and $j: \mathcal{T} \hookrightarrow \mathcal{S} \oplus_{1} \mathcal{T}$ which satisfies the following universal property: For every ucp map $\phi: \mathcal{S} \rightarrow \mathcal{R}$ and ucp map $\psi: \mathcal{T} \rightarrow \mathcal{R}$, where $\mathcal{R}$ is an operator system, there exists a unique 
ucp map $\varphi: \mathcal{S} \oplus_{1} \mathcal{T} \rightarrow R$ such that $\varphi(i(s))=\phi(s)$ and $\varphi(j(t))=\psi(t)$ for every $s$ in $\mathcal{S}$ and $t$ in $\mathcal{T}$.

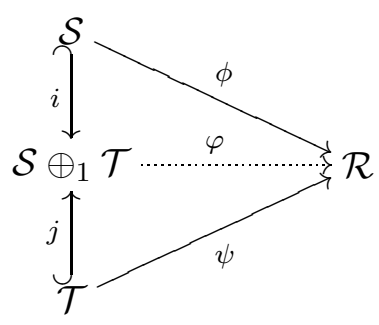

One way to construct this object can be described as follows: Consider $\mathrm{C}^{*}$-algebra free product of $C_{u}^{*}(\mathcal{S}) *_{1} C_{u}^{*}(\mathcal{T})$ amalgamated over the identity. Define $\mathcal{S} \oplus_{1} \mathcal{T}$ as the operator system generated by $\mathcal{S}$ and $\mathcal{T}$ in $C_{u}^{*}(\mathcal{S}) *_{1} C_{u}^{*}(\mathcal{T})$. We leave the verification that this span has the above universal property as an exercise. We also refer to [13, Sec. 3] for a different construction of the coproducts. Below we will obtain coproducts in terms of operator system quotients.

Consider $\mathcal{S} \oplus \mathcal{T}$. Since $(e,-e)$ is a selfadjoint element which is neither positive nor negative, by Theorem [2.3, $J=\operatorname{span}\{(e,-e)\}$ is a kernel in $\mathcal{S} \oplus \mathcal{T}$ (in fact it is a null subspace and hence a completely proximinal kernel by Proposition 2.4). So we have a quotient operator system $(\mathcal{S} \oplus \mathcal{T}) / J$. Note that in the quotient we have

$$
(e, e)+J=(2 e, 0)+J=(0,2 e)+J .
$$

Consider $i: \mathcal{S} \rightarrow \mathcal{S} \oplus \mathcal{T} / J$ by $s \mapsto(2 s, 0)+J$. We claim that $i$ is a unital complete order isomorphism. Clearly it is unital and completely positivity follows from the fact that it can be written as a composition of cp maps, namely $\mathcal{S} \rightarrow \mathcal{S} \oplus \mathcal{T}, s \mapsto(2 s, 0)$ and the quotient map. Now suppose that the image of $\left(s_{i j}\right) \in M_{n}(\mathcal{S})$ is positive. That is, $\left(\left(2\left(s_{i j}, 0\right)+J\right)\right)$ is positive in $M_{n}(\mathcal{S} \oplus \mathcal{T} / J)$. Since $J$ is completely proximinal there are scalars $\alpha_{i j}$ such that $\left(\left(2 s_{i j}+\alpha_{i j} e,-\alpha_{i j} e\right)\right)$ is positive in $M_{n}(\mathcal{S} \oplus \mathcal{T})$. Note that this forces $\left(-\alpha_{i j} e\right)$ to be positive in $M_{n}(\mathcal{T})$. So we have that $\left(2 s_{i j}+\alpha_{i j} e\right)+\left(-\alpha_{i j} e\right)=2\left(s_{i j}\right)$ must be positive in $M_{n}(\mathcal{S})$. Hence $\left(s_{i j}\right)$ is positive and it follows that $i$ is a complete order isomorphism.

Similarly $j: \mathcal{T} \rightarrow \mathcal{S} \oplus \mathcal{T} / J, t \mapsto(0,2 t)+J$ is also a unital complete order isomorphism. Finally let $\phi: \mathcal{S} \rightarrow \mathcal{R}$ and $\psi: \mathcal{T} \rightarrow \mathcal{R}$ be ucp maps. Consider $\varphi: \mathcal{S} \oplus \mathcal{T} / J \rightarrow R$ given by $\varphi((s, t)+J)=(\phi(s)+\psi(t)) / 2$. It is elementary to check $\varphi$ is ucp, $\varphi(i(\cdot))=\phi$ and $\varphi(j(\cdot))=\psi$. Consequently with the above mentioned inclusions we have

$$
\mathcal{S} \oplus_{1} \mathcal{T}=\mathcal{S} \oplus \mathcal{T} / \operatorname{span}\{(e,-e)\} .
$$

We also remark that $C_{u}^{*}\left(\mathcal{S} \oplus_{1} \mathcal{T}\right)=C_{u}^{*}(\mathcal{S}) *_{1} C_{u}^{*}(\mathcal{T})$, which in fact follows from the universal property of the coproduct of operator systems and unital free products of $\mathrm{C}^{*}$-algebras. It is also clear that when $\mathcal{S}$ and $\mathcal{T}$ are finite dimensional then $\operatorname{dim}\left(\mathcal{S} \oplus_{1} \mathcal{T}\right)=\operatorname{dim}(\mathcal{S})+\operatorname{dim}(\mathcal{T})-1$.

The lifting property is preserved under coproducts:

Proposition 8.1. The following are equivalent for finite dimensional operator systems $\mathcal{S}$ and $\mathcal{T}:$

(1) $\mathcal{S}$ and $\mathcal{T}$ have the lifting property.

(2) $\mathcal{S} \oplus_{1} \mathcal{T}$ has the lifting property.

Proof. Suppose $\mathcal{S} \oplus_{1} \mathcal{T}$ has the lifting property. Let $\phi: \mathcal{S} \rightarrow \mathcal{A} / I$ be a ucp map where $I \subset \mathcal{A}$ is a $\mathrm{C}^{*}$-algebra, ideal couple. Suppose $f$ is a state on $\mathcal{T}$ and set $\psi: \mathcal{T} \rightarrow \mathcal{A} / I$ by $\psi=f(\cdot)(e+I)$. By using the universal property of $\mathcal{S} \oplus_{1} \mathcal{T}$ we obtain a ucp map $\varphi: \mathcal{S} * \mathcal{T} \rightarrow \mathcal{A} / I$. For simplicity 
we will identify the $\mathcal{S}$ and $\mathcal{T}$ with their canonical images in $\mathcal{S} \oplus_{1} \mathcal{T}$. Clearly a ucp lift of $\varphi$ on $\mathcal{A}$ is a ucp lift of $\phi$ when restricted to $\mathcal{S}$. Thus $\mathcal{S}$ has osLLP. A similar argument shows that $\mathcal{T}$ has the same property.

Conversely suppose $\mathcal{S}$ and $\mathcal{T}$ have the lifting property. Let $\varphi: \mathcal{S} \oplus_{1} \mathcal{T} \rightarrow \mathcal{A} / I$ be a ucp map. Again we will identify the $\mathcal{S}$ and $\mathcal{T}$ with their canonical images in $\mathcal{S} \oplus_{1} \mathcal{T}$. Let $\phi: \mathcal{S} \rightarrow \mathcal{A}$ be a ucp lift of $\left.\varphi\right|_{\mathcal{S}}$, the restriction of $\varphi$ on $\mathcal{S}$. Similarly let $\psi$ be the ucp lift of $\left.\varphi\right|_{\mathcal{T}}$. Finally by using the universal property of $\mathcal{S} \oplus_{1} \mathcal{T}$ let $\tilde{\varphi}$ be the ucp map from $\mathcal{S} \oplus_{1} \mathcal{T}$ into $\mathcal{A}$ associated with $\phi$ and $\psi$. It is elementary to see that $\tilde{\varphi}$ is a lift of $\varphi$. This finishes the proof.

Recall that we define $\mathcal{S}_{n}$ as the operator system generated by the unitary generator of $C^{*}\left(\mathbb{F}_{n}\right)$, that is,

$$
\mathcal{S}_{n}=\operatorname{span}\left\{g_{1}, \ldots, g_{n}, e, g_{1}^{*}, \ldots, g_{n}^{*}\right\} \subset C^{*}\left(F_{n}\right) .
$$

We remind the reader that $\mathcal{S}_{n}$ can also be considered as the universal operator system generated by $n$ contractions as it satisfies the following universal property: Every function $f:\left\{g_{i}\right\}_{i=1}^{n} \rightarrow \mathcal{T}$ with $\left\|f\left(g_{i}\right)\right\| \leq 1$ extends uniquely to a ucp map $\varphi_{f}: S_{n} \rightarrow \mathcal{T}$ (in an obvious way).

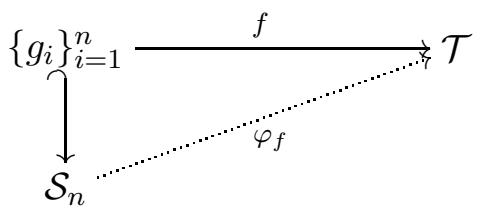

It is easy to see that $\mathcal{S}_{n}$ is naturally included in $\mathcal{S}_{n+k}$ where the inclusion is given by the map $g_{i} \mapsto g_{i}$ for $i=1, \ldots, n$. In a similar way, $\mathcal{S}_{k}$ can also be represented in $\mathcal{S}_{n+k}$ via the map $g_{i} \mapsto g_{n+i}$ for $i=1, \ldots, k$. Thus, there is a map from $\mathcal{S}_{n} \oplus_{1} \mathcal{S}_{k}$ to $\mathcal{S}_{n+k}$. The following result states that this natural map is a complete order isomorphism. We skip its elementary proof. In fact, it is easy to show that $\mathcal{S}_{n+k}$ satisfies the universal property that $\mathcal{S}_{n} \oplus_{1} \mathcal{S}_{k}$ has.

Lemma 8.2. $\mathcal{S}_{n} \oplus_{1} \mathcal{S}_{k}=\mathcal{S}_{n+k}$.

Example. We wish to show that $\mathcal{S}_{1}=\operatorname{span}\left\{g, e, g^{*}\right\} \subset C^{*}\left(\mathbb{F}_{1}\right)$ is $\mathrm{C}^{*}$-nuclear. This is based on Sz.-Nagy's dilation theorem (see [33, Thm. 1.1], e.g.): If $T \in B(H)$ is a contraction then there is a Hilbert space $K$ containing $H$ as a subspace and a unitary operator $U$ in $B(K)$ such that $T^{n}=\left.P_{H} U^{n}\right|_{H}$ for every positive $n$. Of course, by taking the adjoint, we also have that $\left(T^{*}\right)^{n}=\left.P_{H}\left(U^{*}\right)^{n}\right|_{H}$ for every positive $n$. This means that there is a ucp map defined from $C^{*}\left(\mathbb{F}_{1}\right)$ into $C^{*}\left\{I, T, T^{*}\right\}$, the $\mathrm{C}^{*}$-algebra generated by $T$ in $B(H)$, which is given by the compression of the unital $*$-homomorphism extending the representation $g \mapsto U$. That is, the map $\gamma_{T}$ defined from $C^{*}\left(\mathbb{F}_{1}\right)$ into $B(H)$ given by $g^{n} \mapsto T^{n}, e \mapsto I$ and $g^{-n} \mapsto\left(T^{*}\right)^{n}$ is ucp. Now we wish to show that $\mathcal{S}_{1} \otimes_{\max } \mathcal{A} \subset C^{*}\left(\mathbb{F}_{1}\right) \otimes_{\max } \mathcal{A}$ for every $\mathcal{A}$. Let $\varphi: \mathcal{S}_{1} \otimes_{\max } \mathcal{A} \rightarrow B(K)$ be a ucp map. Then by Proposition [3.5, There is a Hilbert space $K_{1}$ containing $K$ as a subspace and ucp maps $\phi: \mathcal{S}_{1} \rightarrow B\left(K_{1}\right)$ and $\psi: \mathcal{A} \rightarrow B\left(K_{1}\right)$ with commuting ranges such that $\varphi=\left.P_{K} \phi \cdot \psi\right|_{K}$. Note that $\phi(g)$ must be a contraction. The map $\gamma_{\phi(g)}$ is a ucp extension of $\phi$ on $C^{*}\left(\mathbb{F}_{1}\right)$. Clearly $\gamma_{\phi(g)}$ and $\psi$ have commuting ranges. Thus $\left.P_{K} \gamma_{\phi(g)} \cdot \psi\right|_{K}$ is a ucp extension of $\varphi$ on $C^{*}\left(\mathbb{F}_{1}\right) \otimes_{\max } \mathcal{A}$. In conclusion we have that every ucp map defined from $\mathcal{S}_{1} \otimes_{\max } \mathcal{A}$ into a $B(K)$ extends to a ucp map on $C^{*}\left(\mathbb{F}_{1}\right) \otimes_{\max } \mathcal{A}$. This is enough to conclude that $\mathcal{S}_{1} \otimes_{\max } \mathcal{A} \subset C^{*}\left(\mathbb{F}_{1}\right) \otimes_{\max } \mathcal{A}$. It is well known that $C^{*}\left(\mathbb{F}_{1}\right)=C^{*}(\mathbb{Z})=C(\mathbb{T})$ (see [40], e.g.) and the $\mathrm{C}^{*}$-algebra of continuous functions on a compact set is nuclear (see [33], e.g.). Since $\mathcal{S}_{1} \otimes_{\min } \mathcal{A} \subset C^{*}\left(\mathbb{F}_{1}\right) \otimes_{\min } \mathcal{A}$ and $C^{*}\left(\mathbb{F}_{1}\right)$ is nuclear we conclude that $\mathcal{S}_{1}$ is $\mathrm{C}^{*}$-nuclear.

Question 8.3. In the previous example we have shown that the three dimensional operator system $\operatorname{span}\left\{1, z, z^{*}\right\} \subset C(\mathbb{T})$, where $z$ is the coordinate function, is $\mathrm{C}^{*}$-nuclear. In general, if $X$ is a compact set then is every three dimensional operator subsystem $\operatorname{span}\left\{1, f, f^{*}\right\} \subset C(X)$ 
$\mathrm{C}^{*}$-nuclear? In fact by using spectral theorem it is enough to consider the case when $X$ is subset of $\{z:|z| \leq 1\}$. So, when this subset is the unit circle then the answer is affirmative.

Since the Kirchberg Conjecture (KC) is equivalent to the statement that $S_{2}$ has DCEP it is natural to raise the following questions:

Question 8.4. Suppose $\mathcal{S}$ and $\mathcal{T}$ are two finite dimensional operator systems with DCEP. Does $\mathcal{S} \oplus_{1} \mathcal{T}$ have DCEP?

Question 8.5. Suppose $\mathcal{S}$ and $\mathcal{T}$ are two finite dimensional $\mathrm{C}^{*}$-nuclear operator systems. Does $\mathcal{S} \oplus_{1} \mathcal{T}$ have DCEP?

Results: An affirmative answer to the Question 8.4 implies an affirmative answer to the KC. This follows from the fact that $\mathcal{S}_{2}=\mathcal{S}_{1} \oplus_{1} \mathcal{S}_{1}$ and $\mathcal{S}_{1}$ is $\mathrm{C}^{*}$-nuclear, in particular it has DCEP. On the other hand Question 8.5 is equivalent to the KC. First suppose that KC is true. If $\mathcal{S}$ and $\mathcal{T}$ are $\mathrm{C}^{*}$-nuclear operator systems then, in particular, they have the lifting property and so $\mathcal{S} \oplus_{1} \mathcal{T}$ has the lifting property. Since we assumed KC, by using Theorem 5.10, $\mathcal{S} \oplus_{1} \mathcal{T}$ must have DCEP. Conversely if we suppose that Question 8.5 is true then in particular $\mathcal{S}_{2}=\mathcal{S}_{1} \oplus_{1} \mathcal{S}_{1}$ has DCEP.

\section{K-MINIMALITY AND K-MAXIMALITY}

In this section we review k-minimality and k-maximality in the category of operator systems introduced by Xhabli in [44]. This theory and a similar construction in the category of operator spaces are used extensively in the understanding of entanglement breaking maps and separability problems in quantum information theory (44], [45] and [19], e.g.). Our interest in k-minimality and k-maximality arises from their compatiblity with exactness and the lifting property which will be apparent in this section. We start with the following observation:

Proposition 9.1. Let $\varphi: \mathcal{S} \rightarrow B(H)$ be a linear map. Then $\varphi$ is k-positive if and only if there is a unital $k$-positive map $\psi: \mathcal{S} \rightarrow B(H)$ and $R \geq 0$ in $B(H)$ such that $\varphi=R \psi(\cdot) R$.

Proof. We will show only the non-trivial direction. Let $\varphi: \mathcal{S} \rightarrow B(H)$ be a $k$-positive map. We assume that $\varphi(e)=A$ satisfies $0 \leq A \leq I$, where $I$ is the identity in $B(H)$. For any $\epsilon>0$ let $\varphi_{\epsilon}: \mathcal{S} \rightarrow B(H)$ be the map defined by $\varphi_{\epsilon}=(A+\epsilon I)^{-1 / 2} \varphi(\cdot)(A+\epsilon I)^{-1 / 2}$. Since $B(\mathcal{S}, B(H))$ is a dual object, which arises from the fact that $B(H)$ is dual of a Banach space, the net $\left\{\varphi_{\epsilon}\right\}$ has a $w^{*}$-limit, say $\psi$. First note that $\psi$ is unital. Indeed, $\varphi_{\epsilon}(e)=A(A+\epsilon I)^{-1}$ converges to the identity $I$ in the $w^{*}$-topology of $B(H)$. Consequently $\psi$ is unital. We also claim that $\psi$ is $k$-positive. To see this let $\left(s_{i j}\right)$ be positive in $M_{k}(\mathcal{S})$. Since $\varphi_{\epsilon}$ is $k$-positive we have that $\left(\varphi_{\epsilon}\left(s_{i j}\right)\right)$ is positive in $M_{k}(B(H))$. The weak convergence $\varphi_{\epsilon} \rightarrow \psi$ ensures that, for fixed $i, j, \varphi_{\epsilon}\left(s_{i j}\right)$ has a limit in the $w^{*}$-topology of $B(H)$ which is necessarily $\psi\left(s_{i j}\right)$. Now the result follows from the fact that positives cones are closed in the $w^{*}$-topology of $B(H)$. Finally we claim that $\varphi=A^{1 / 2} \psi(\cdot) A^{1 / 2}$. Indeed this follows from the uniqueness of the $w^{*}$ limit in $B(\mathcal{S}, B(H))$. In fact we have that $A^{1 / 2} \varphi_{\epsilon}(\cdot) A^{1 / 2}$ converges to $A^{1 / 2} \psi(\cdot) A^{1 / 2}$. On the other hand for fixed $s$ in $\mathcal{S}, A^{1 / 2} \varphi_{\epsilon}(s) A^{1 / 2}$ converges to $\varphi(s)$ (in the $w^{*}$-topology of $B(H)$ ). So the proof is done.

Corollary 9.2. The following properties of an operator system $\mathcal{S}$ are equivalent:

(1) Every k-positive map defined from $\mathcal{S}$ into an operator system is $\mathrm{cp}$.

(2) Every unital k-positive map defined from $\mathcal{S}$ into an operator system is cp. 
Before getting started with the k-minimality and k-maximality we also recall the following result (see Thm. 6.1 of [33]).

Lemma 9.3. Suppose $\phi: \mathcal{S} \rightarrow M_{k}$ is a linear map. Then $\phi$ is k-positive if and only if it is completely positive.

Following Xhabli [4], for an operator system $\mathcal{S}$ we define the k-minimal cone structure as follows:

$$
C_{n}^{k-m i n}=\left\{\left(s_{i j}\right) \in M_{n}(\mathcal{S}):\left(\phi\left(s_{i j}\right)\right) \geq 0 \text { for every ucp } \phi: \mathcal{S} \rightarrow M_{k}\right\} .
$$

By considering Proposition 9.1 one can replace ucp by cp in this definition. Now, the $*-$ vector space $\mathcal{S}$ together with the matricial cone structure $\left\{C_{n}^{k-\min }\right\}_{n=1}^{\infty}$ and the unit $e$ form an operator system which is called the $k$-minimal operator system structure generated by $\mathcal{S}$ and denoted by $\mathrm{OMIN}_{k}(\mathcal{S})$. We refer [44, Section 2.3] for the proof of these results and we remark that $\mathrm{OMIN}_{k}(\mathcal{S})$ is named as super $k$-minimal structure so we drop the term "super" in this paper. Roughly speaking $\operatorname{OMIN}_{k}(\mathcal{S})$ is (possibly) a new operator system whose positive cones coincide with the positive cones of $\mathcal{S}$ up to the $k^{t h}$ level and after the $k^{\text {th }}$ level they are the largest cones so that the total matricial cone structure is still an operator system. Note that larger cones generate smaller canonical operator space structure so this construction is named the k-minimal structure. We list a couple of remarkable results from [44]:

Theorem 9.4 (Xhabli). Suppose $\mathcal{S}$ is an operator system and $k$ is a fixed number. Then:

(1) $\mathrm{OMIN}_{k}(\mathcal{S})$ can be represented in $M_{k}(C(X))$ for some compact space $X$.

(2) If $\varphi: \mathcal{T} \rightarrow \operatorname{OMIN}_{k}(\mathcal{S})$ is a k-positive map then $\varphi$ is completely positive.

(3) The identity id: $\operatorname{OMIN}_{k}(\mathcal{S}) \rightarrow \mathcal{S}$ is k-positive.

(4) For any $m \leq k$ the identities $\mathcal{S} \rightarrow \operatorname{OMIN}_{k}(\mathcal{S}) \rightarrow \operatorname{OMIN}_{m}(\mathcal{S})$ are completely positive.

Lemma 9.5. Let $\mathcal{S}$ be an operator system. Then $\mathcal{S}=\operatorname{OMIN}_{k}(\mathcal{S})$ if and only if every $k$ positive map defined from an operator system into $\mathcal{S}$ is completely positive.

Proof. One direction follows from the above result of Xhabli. Conversely, suppose that every k-positive map defined into $\mathcal{S}$ is cp. This, in particular, implies that the identity $i d: \operatorname{OMIN}_{k}(\mathcal{S}) \rightarrow \mathcal{S}$, which is k-positive, is cp. Since the inverse of this map is also cp it follows that $\mathcal{S}=\mathrm{OMIN}_{k}(\mathcal{S})$.

Let $\mathcal{S}$ be an operator system and $k$ be a fixed natural number. To define the k-maximal structure we first consider the following cones:

$$
\begin{array}{r}
D_{n}^{k-\max }=\left\{A^{*} D A: A \in M_{m k, n} \text { and } D=\operatorname{diagonal}\left(D_{1}, \ldots, D_{m}\right)\right. \\
\text { where } \left.D_{i} \in M_{k}(\mathcal{S})^{+} \text {for } i=1, \ldots, m\right\} .
\end{array}
$$

$\left\{D_{n}^{k-\max }\right\}$ forms a strict compatible matricial order structure on $\mathcal{S}$ and $e$ is an matricial order unit. However, $e$ fails to be Archimedean and to resolve this problem we use the Archimedeanization process (see [37]):

$$
C_{n}^{k-\max }=\left\{\left(s_{i j}\right) \in M_{n}(\mathcal{S}):\left(s_{i j}\right)+\epsilon e_{n} \in D_{n}^{k-\max } \text { for every } \epsilon>0\right\} .
$$

Note that $D_{n}^{k-\max } \subset C_{n}^{k-\max }$. The $*$-vector space $\mathcal{S}$ together with the matricial cone structure $\left\{C_{n}^{k-\max }\right\}_{n=1}^{\infty}$ and the unit $e$ form an operator system which is called $k$-maximal operator system structure generated by $\mathcal{S}$ and denoted by $\operatorname{OMAX}_{k}(\mathcal{S})$. For related proof we refer [44, Sec. 2.3.]. (We again drop the term "super".) The $\operatorname{OMAX}_{k}(\mathcal{S})$ is (possibly) a new operator system structure on the $*$-vector space $\mathcal{S}$ such that the matricial cones coincide with the matricial cones of the operator system $\mathcal{S}$ up to $k^{t h}$-level and after $k$, the cones are the smallest possible cones such a way that the total structure makes $\mathcal{S}$ an operator system with unit $e$. 
Theorem 9.6 (Xhabli). Let $\mathcal{S}$ be an operator system and $k$ be a fixed number. Then:

(1) Every $k$-positive map defined from $\operatorname{OMAX}_{k}(\mathcal{S})$ into an operator system is completely positive.

(2) The identity id $: \mathcal{S} \rightarrow \operatorname{OMAX}_{k}(\mathcal{S})$ is $k$-positive.

(3) For any $m \leq k$ the identities $\operatorname{OMAX}_{m}(\mathcal{S}) \rightarrow \operatorname{OMAX}_{k}(\mathcal{S}) \rightarrow \mathcal{S}$ are completely positive.

The proof of the following lemma is similar to Lemma 9.5 so we skip it.

Lemma 9.7. Let $\mathcal{S}$ be an operator system. Then $\mathcal{S}=\operatorname{OMAX}_{k}(\mathcal{S})$ if and only if every $k$-positive map defined from $\mathcal{S}$ into another operator system is completely positive.

After these preliminary results we are ready to examine the role of k-minimality and the k-maximality in the nuclearity theory. We start with the following easy observation:

Lemma 9.8. $\operatorname{OMIN}_{k}(\mathcal{S})$ is exact for any operator system $\mathcal{S}$ and $k$.

Proof. Recall that $\mathrm{OMIN}_{k}(\mathcal{S})$ can be represented in $M_{k}(C(X))$ for some compact space $X$. Note that $M_{k}(C(X))$ is a nuclear $\mathrm{C}^{*}$-algebra and consequently it is (min,max)-nuclear operator system. Clearly ( $\min , \max )$-nuclearity implies (min,el)-nuclearity (equivalently exactness) and, by Proposition 4.10, exactness passes to operator subsystems so we have that $\operatorname{OMIN}_{k}(\mathcal{S})$ is exact.

Note that if $\mathcal{S}$ is a finite dimensional operator system then a faithful state on $\mathcal{S}$ still has the same property when $\mathcal{S}$ is equipped with $\mathrm{OMIN}_{k}$ or $\mathrm{OMAX}_{k}$ structure. Keeping this observation in mind we are ready to state:

Theorem 9.9. Let $\mathcal{S}$ be a finite dimensional operator system. Then we have the unital complete order isomorphisms

$$
\mathrm{OMIN}_{k}(\mathcal{S})^{d}=\operatorname{OMAX}_{k}\left(\mathcal{S}^{d}\right) \quad \text { and } \operatorname{OMAX}_{k}(\mathcal{S})^{d}=\operatorname{OMIN}_{k}\left(\mathcal{S}^{d}\right) .
$$

Proof. We only prove the fist equality. The second equality follows from the first one if we replace $\mathcal{S}$ by $\mathcal{S}^{d}$ and take the dual of both side. To show the first one we set $\mathcal{R}=\operatorname{OMIN}_{k}(\mathcal{S})$ and we will first prove the following: Whenever $\varphi: \mathcal{R}^{d} \rightarrow \mathcal{T}$ is a k-positive map then $\varphi$ is cp. So by using Lemma 9.7 we conclude that $\mathcal{R}^{d}=\operatorname{OMAX}_{k}\left(\mathcal{R}^{d}\right)$. Assume for a contradiction that there is a k-positive map $\varphi: \mathcal{R}^{d} \rightarrow \mathcal{T}$ which is not cp. Clearly we may assume that $\mathcal{T}$ is finite dimensional. (If not we can consider an operator subsystem of $\mathcal{T}$ containing the image of $\varphi$.) Now by using Lemma 1.4 we have that $\varphi^{d}: \mathcal{T}^{d} \rightarrow \mathcal{R}$ is a k-positive map but it is not cp. This is a contradiction as Lemma 9.5 requires that $\varphi$ is a cp map. Thus $\mathcal{R}^{d}=\operatorname{OMAX}_{k}\left(\mathcal{R}^{d}\right)$. Next we show that $\operatorname{OMAX}_{k}\left(\mathcal{R}^{d}\right)=\operatorname{OMAX}_{k}\left(\mathcal{S}^{d}\right)$ which finishes the proof. To see this note that the identity $i d: \mathcal{S} \rightarrow \mathcal{R}$ is cp and its inverse is $k$-positive. This implies that $i d^{d}: \mathcal{R}^{d} \rightarrow \mathcal{S}$ is cp and its inverse is $k$-positive. (We skip the elementary proof of the fact that $\left(\varphi^{d}\right)^{-1}=\left(\varphi^{-1}\right)^{d}$.) Thus up to $k^{\text {th }}$ level $\mathcal{R}^{d}$ and $\mathcal{S}^{d}$ are order isomorphic. Hence $\operatorname{OMAX}_{k}\left(\mathcal{R}^{d}\right)=\operatorname{OMAX}_{k}\left(\mathcal{S}^{d}\right)$. Finally by using the observation that we mentioned before the theorem we may assume that this identification is also unital.

Lemma 9.10. Suppose $\mathcal{S}$ is a finite dimensional operator system. Then $\operatorname{OMAX}_{k}(\mathcal{S})$ has the lifting property for any natural number $k$.

Proof. Lemma 9.8 states that $\operatorname{OMIN}_{k}\left(\mathcal{S}^{d}\right)$ is exact. By the above theorem we see that $\operatorname{OMIN}_{k}\left(\mathcal{S}^{d}\right)^{d}=\operatorname{OMAX}_{k}(\mathcal{S})$ and by using Theorem 6.6 we conclude that this dual has the lifting property.

We are now ready to establish a weaker lifting property: 
Theorem 9.11. Every finite dimensional operator system $\mathcal{S}$ has the $k$-lifting property (for every $k$ ) in the sense that whenever $I$ is an ideal in a unital $C^{*}$-algebra $\mathcal{A}$ and $\varphi: \mathcal{S} \rightarrow \mathcal{A} / I$ is a ucp map then, for every $k$, there exists a unital k-positive map $\tilde{\varphi}: \mathcal{S} \rightarrow \mathcal{A}$ such that $q \circ \tilde{\varphi}=\varphi$ where $q: \mathcal{A} \rightarrow \mathcal{A} / I$ is the quotient map.

Proof. Let $\varphi: \mathcal{S} \rightarrow \mathcal{A} / I$ be a ucp map. When $\mathcal{S}$ is equipped with $\mathrm{OMAX}_{k}$ structure $\varphi$ remains to be a ucp map. By Lemma $9.10 \operatorname{OMAX}_{k}(\mathcal{S})$ has the lifting property so $\varphi$ lifts to a cp map $\tilde{\varphi}$ on $\mathcal{A}$ which can taken to be unital. Now when we consider $\tilde{\varphi}$ as a map defined from $\mathcal{S}$ it is $k$-positive. This completes the proof.

We want to remark that if $\mathcal{S}$ is a finite dimensional operator system then the $k$-lifting property, which $\mathcal{S}$ has for every $k$, does not imply the lifting property. In [35, Theorem 3.3.] it was shown that there is a five dimensional operator subsystem of the Calkin algebra $\mathbb{B} / \mathbb{K}$ such that the inclusion does not have a ucp lift (or cp lift) on $\mathbb{B}$. In the next section we will see that even $M_{2} \oplus M_{2}$ has a five dimensional operator system that does not have the lifting property. For three dimensional operator systems a similar problem turns out to be equivalent to the Smith Ward problem which we will study in Section [11.

Corollary 9.12. Let $\mathcal{S}$ be a finite dimensional operator system, $\mathcal{A}$ be a $C^{*}$-algebra and $I$ be an ideal. Then every $k$-positive map $\mathcal{S} \rightarrow \mathcal{A} / I$ has a $k$-positive lifting to $\mathcal{A}$. If $\varphi$ is unital one can take the lift unital too.

Proof. If we equip $\mathcal{S}$ with $\operatorname{OMAX}_{k}$ structure then $\varphi$ is completely positive. Since $\operatorname{OMAX}_{k}(\mathcal{S})$ has the lifting property, by using Remark 4.5. $\varphi$ can be lifted as a completely positive map on $\mathcal{A}$. If $\varphi$ is unital one can pick the lift unital as well. Now when $\mathcal{S}$ is considered with its initial structure this lift is $k$-positive.

Corollary 9.13. Let $X$ be a finite dimensional operator space, $\mathcal{A}$ be a unital $C^{*}$-algebra and $I$ be an ideal in $\mathcal{A}$. Then every completely contractive (cc) map $\phi: X \rightarrow \mathcal{A} / I$ has a $k$-contractive lift on $\mathcal{A}$ for every $k$.

Proof. Recall that the universal operator system $\mathcal{R}_{X} \supset X$ has the property that every cc map defined from $X$ into an operator system extends uniquely to a ucp map. Now, $\phi$ extends to a ucp map $\varphi: \mathcal{R}_{X} \rightarrow \mathcal{A} / I$. By the above corollary this map has a unital $2 k$-positive lift on $\mathcal{A}$. Since a unital $2 k$-positive map is $k$-contractive, the restriction of this lift on $X$ has the desired property.

\section{Quotients of the Matrix Algebra $M_{n}$}

In this section we obtain new proofs of some of the results of [10] and discuss some new formulations of the Kirchberg Conjecture (KC) in terms of operator system quotients of the matrix algebras. The duality and the quotient theory when applied to some special operator subsystems of $M_{n}$ raise difficult stability problems which will be apparent in this section. We will also consider the problem about the minimal and the maximal tensor product of three copies of $C^{*}\left(\mathbb{F}_{\infty}\right)$ from an operator system perspective.

Recall from Example 6.9 that we define $J_{n} \subset M_{n}$ as the diagonal matrices with 0 trace. As we pointed out, $J_{n}$ is a null subspace of $M_{n}$ and consequently, by Proposition 2.4, it is a completely proximinal kernel. (Also recall that $M_{n} / J_{n}$ has the lifting property.) However, with the following result of Farenick and Paulsen we directly see that $J_{n}$ is a kernel and, moreover, we obtain an identification of $M_{n} / J_{n}$ as well as its enveloping $\mathrm{C}^{*}$-algebra. 
As usual $C^{*}\left(\mathbb{F}_{n}\right)$ stands for the full $\mathrm{C}^{*}$-algebra of the free group $\mathbb{F}_{n}$ on $n$ generators, say $g_{1}, \ldots, g_{n}$. Let $\mathcal{W}_{n}$ be the operator subsystem of $C^{*}\left(\mathbb{F}_{n}\right)$ given by

$$
\mathcal{W}_{n}=\left\{g_{i} g_{j}^{*}: 1 \leq i, j \leq n\right\} .
$$

We are now ready to establish the connection between these operator systems given in [10]. As usual $\left\{E_{i j}\right\}$ denotes the standard matrix units for $M_{n}$. Consider $\varphi: M_{n} \rightarrow \mathcal{W}_{n}$ given by $\varphi\left(E_{i j}\right)=g_{i} g_{j}^{*} / n$. Then

Theorem 10.1 (Farenick, Paulsen). The above map $\varphi: M_{n} \rightarrow \mathcal{W}_{n}$ is a quotient map with kernel $J_{n}$. That is, the induced map $\bar{\varphi}: M_{n} / J_{n} \rightarrow \mathcal{W}_{n}$ is a bijective unital complete order isomorphism. Moreover, $C_{e}^{*}\left(M_{n} / J_{n}\right)=C^{*}\left(\mathbb{F}_{n-1}\right)$.

Now we are ready to state:

Theorem 10.2. The following are equivalent:

(1) KC has an affirmative answer.

(2) $M_{3} / J_{3}$ has DCEP.

(3) $M_{3} / J_{3} \otimes_{\min } M_{3} / J_{3}=M_{3} / J_{3} \otimes_{c} M_{3} / J_{3}$.

Proof. Example 6.9 states that $M_{3} / J_{3}$ has the lifting property. So if we assume (1) then, by Theorem 5.10, $M_{3} / J_{3}$ has DCEP. This proves that (1) implies (2). To see that (2) implies (3) we recall that the lifting property is characterized by (min,er)-nuclearity. Thus we readily have that $M_{3} / J_{3} \otimes_{\min } M_{3} / J_{3}=M_{3} / J_{3} \otimes_{e r} M_{3} / J_{3}$. Now, by our assumption, $M_{3} / J_{3}$ has DCEP, equivalently, (el,c)-nuclearity. Now, applying this to $M_{3} / J_{3}$ on the right hand side, we have that $M_{3} / J_{3} \otimes_{e r} M_{3} / J_{3}=M_{3} / J_{3} \otimes_{c} M_{3} / J_{3}$. Thus, (2) implies (3). We finally show that (3) implies (1). In fact, $M_{3} / J_{3}$ contains enough unitaries in its enveloping $\mathrm{C}^{*}$-algebra, namely, $C^{*}\left(\mathbb{F}_{2}\right)$ (see Section 5 for the related definition). This simply follows from the fact that $\mathcal{W}_{3}$ is linear span of unitaries, thus, it contains enough unitaries in the $\mathrm{C}^{*}$-algebra generated by itself (in $C^{*}\left(\mathbb{F}_{3}\right)$ ). So, by Proposition $\left[5.6\right.$, this $\mathrm{C}^{*}$-algebra must be coincides with its enveloping $\mathrm{C}^{*}$-algebra. Now, by identifying $M_{n} / J_{n}$ with $\mathcal{W}_{n}$, we conclude that $M_{3} / J_{3}$ contains enough unitaries in its enveloping $\mathrm{C}^{*}$-algebra, namely, $C^{*}\left(\mathbb{F}_{2}\right)$. Thus assuming (3), by Corollary [5.8, we have that $C^{*}\left(\mathbb{F}_{2}\right) \otimes_{\min } C^{*}\left(\mathbb{F}_{2}\right)=C^{*}\left(\mathbb{F}_{2}\right) \otimes_{\max } C^{*}\left(\mathbb{F}_{2}\right)$. Thus (3) implies (1).

We remark that Theorem 5.2. of [10] states that if $M_{n} / J_{n} \otimes_{\min } M_{n} / J_{n}=M_{n} / J_{n} \otimes_{\max }$ $M_{n} / J_{n}$ for every $n$ then it follows that KC has an affirmative answer.

Question 10.3. Is $M_{n} / J_{n} \otimes_{c} M_{n} / J_{n}=M_{n} / J_{n} \otimes_{\max } M_{n} / J_{n}$ for every $n$ ? What about $n=3$ ?

Recall that we define $S_{n}$ as the operator subsystem of $C^{*}\left(\mathbb{F}_{n}\right)$ which contains the unitary generators. More precisely, $\mathcal{S}_{n}=\left\{g_{1}, \ldots, g_{n}, e, g_{1}^{*}, \ldots, g_{n}^{*}\right\}$. Another important operator subsystem of $M_{n}$, which is related to $\mathcal{S}_{n}$, is the tridiagonal matrices $T_{n}$. We define

$$
T_{n}=\left\{A=\left(a_{i j}\right) \in M_{n}: a_{i j}=0 \text { if }|i-j| \geq 1\right\} .
$$

The study on the nuclearity properties of these operator systems goes back to [22]. In Theorem 5.16 it was shown that $T_{3}$ is $\mathrm{C}^{*}$-nuclear (i.e. (min,c)-nuclear). In general, Proposition 6.11 states that if $\mathcal{S}$ is an operator subsystem of $M_{n}$ associated with a chordal graph $G$ then $\mathcal{S}$ is $\mathrm{C}^{*}$-nuclear. We refer to Section 5 of $[22$ for related definitions and discussions. Since $T_{n}$ is associated with the chordal graph (over vertices $\{1,2, \ldots, n\}$ )

$$
\{(1,1),(1,2),(2,1),(2,2),(2,3),(3,2),(3,3),(3,4), \ldots,(n, n)\}
$$

we have that

Proposition 10.4. $T_{n}$ is $C^{*}$-nuclear for every $n$. 
As we mentioned at the end of Section 3, a finite dimensional operator system is (c,max)nuclear if and only if it completely order isomorphic to a $\mathrm{C}^{*}$-algebra. Consequently, for an operator system which is not a $\mathrm{C}^{*}$-algebra, such as $T_{n}, \mathrm{C}^{*}$-nuclearity is the highest nuclearity that one should expect.

Since $J_{n}$, the diagonal $n \times n$ matrices with 0 trace, is a null subspace of $T_{n}$, by Proposition 2.4. it is a completely proximinal kernel. Also note that $\mathrm{C}^{*}$-nuclearity clearly implies the lifting property and so, by Theorem 6.8, we have that $T_{n} / J_{n}$ has lifting property. The following is from [10]:

Theorem 10.5. $T_{n} / J_{n}$ is unitally completely order isomorphic to $\mathcal{S}_{n-1}$. More precisely, the uсp map $\gamma: T_{n} \rightarrow \mathcal{S}_{n-1}$ given by

$$
\begin{array}{ll}
E_{i, i} & \mapsto e / n \text { for } i=1, \ldots, n \\
E_{i, i+1} & \mapsto g_{i} / n \text { for } i=1, \ldots, n-1 \\
E_{i+1, i} & \mapsto g_{i}^{*} / n \text { for } i=1, \ldots, n-1
\end{array}
$$

is a quotient map with kernel $J_{n}$.

This again brings difficult stability problems we have considered in the last section:

Corollary 10.6. The following are equivalent:

(1) KC has an affirmative answer.

(2) For any finite dimensional $C^{*}$-nuclear operator system $\mathcal{S}$ and null subspace $J$ of $\mathcal{S}$ one has $\mathcal{S} / J$ has DCEP.

(3) $T_{n} / J_{n}$ has DCEP for every $n$.

(4) $T_{3} / J_{3}$ has DCEP.

Proof. Since $T_{3} / J_{3}=\mathcal{S}_{2},(1)$ and (4) are equivalent by Theorem 5.10, Also, as we mentioned, $\mathcal{T}_{n} / J_{n}$ has lifting property. So if we assume (1) we must have that $\mathcal{T}_{n} / J_{n}$ has DCEP. (3) implies (4) is clear. Now we need to show that (2) is equivalent to remaining. Clearly (2) implies (4) (or (3)). On the other hand if $\mathcal{S}$ is $\mathrm{C}^{*}$-nuclear then, in particular, it has the lifting property and so, by Theorem 6.8, $\mathcal{S} / J$ has the lifting property. So assuming (1) we must have that this quotient has DCEP.

This corollary indicates that $\mathrm{KC}$ is indeed an operator system quotient problem. DCEP is one of the extensions of WEP from unital $\mathrm{C}^{*}$-algebras to general operator systems. In addition to being equivalent to (el,c)-nuclearity we have seen that it is an important property in the understanding of KC. However, the following definition will allow us to relax DCEP to another property:

Definition 10.7. We say that an operator system $\mathcal{S}$ has property $\mathbb{S}_{2}$ if $\mathcal{S} \otimes_{\min } \mathcal{S}_{2}=\mathcal{S} \otimes_{c} \mathcal{S}_{2}$.

We remark that, for unital $\mathrm{C}^{*}$-algebras, property $\mathbb{S}_{2}$ coincides with WEP. That is, a unital $\mathrm{C}^{*}$-algebra has WEP if and only if it has property $\mathbb{S}_{2}$. This directly follows from Theorem [5.9. It is also worth mentioning that, again for unital $\mathrm{C}^{*}$-algebras, property $\mathbb{S}_{2}$ coincides with property $\mathfrak{W}$ and property $\mathfrak{S}$ in [10]. We refer the reader to Section 3 and 6 in [10] for related definitions. For the operator systems we have that

$$
W E P \quad \Longrightarrow \quad D C E P \quad \Longrightarrow \quad \text { property } \mathbb{S}_{2} \text {. }
$$

We know that DCEP, in general, does not imply WEP. For example if $\mathcal{S}$ is a finite dimensional operator system then WEP is equivalent to $\mathcal{S}$ having the structure of a $\mathrm{C}^{*}$-algebra (which follows from the fact that (el,max)-nuclearity implies (c,max)-nuclearity). On the other hand $T_{n}$ is a $\mathrm{C}^{*}$-nuclear operator system for every $n$ and in particular it has DCEP. So this family 
forms an example that DCEP is weaker than WEP. To see that DCEP implies property $\mathbb{S}_{2}$, let $\mathcal{S}$ be an operator system with DCEP (equivalently (el,c)-nuclearity). Since $\mathcal{S}_{2}$ has the lifting property (i.e. (min,er)-nuclearity) (and keeping in mind that it is written on the right hand side) we have

$$
\mathcal{S} \otimes_{\min } \mathcal{S}_{2}=\mathcal{S} \otimes_{e l} \mathcal{S}_{2}=\mathcal{S} \otimes_{c} \mathcal{S}_{2}
$$

Thus, $\mathcal{S}$ has property $\mathbb{S}_{2}$. However we don't know whether property $\mathbb{S}_{2}$ implies DCEP.

Question 10.8. Does property $\mathbb{S}_{2}$ imply DCEP?

Proposition 10.9. Suppose $\mathcal{S} \otimes_{\tau} \mathcal{T}$ has property $\mathbb{S}_{2}$ (resp. has DCEP) where $\tau$ is any functorial tensor product. Then both $\mathcal{S}$ and $\mathcal{T}$ have property $\mathbb{S}_{2}$ (resp. have DCEP).

Proof. This follows from a very basic principle: The identity on $\mathcal{S}$ factors via ucp maps through $\mathcal{S} \otimes_{\tau} \mathcal{T}$. More precisely, the inclusion $i: \mathcal{S} \rightarrow \mathcal{S} \otimes_{\tau} \mathcal{T}$ given by $s \mapsto s \otimes_{\mathcal{T}}$ is a ucp map. Conversely, if $g$ is a state on $\mathcal{T}$ then $i d \otimes g: \mathcal{S} \otimes_{\tau} \mathcal{T} \rightarrow \mathcal{S} \otimes \mathbb{C} \cong \mathcal{S}$ is again a ucp map such that $(i d \otimes g) \circ i$ is the identity on $\mathcal{S}$. This shows that if $\mathcal{S} \otimes_{\tau} \mathcal{T}$ has DCEP (equivalently (el,c)-nuclearity) then by Lemma $5.2 \mathcal{S}$ has DCEP. Clearly a similar argument shows that $\mathcal{T}$ has the same property. Now suppose that $\mathcal{S} \otimes_{\tau} \mathcal{T}$ has property $\mathbb{S}_{2}$. By using the functoriality of min and c tensor products we have that

$$
\mathcal{S} \otimes_{\min } \mathcal{S}_{2} \stackrel{i \otimes i d}{\longrightarrow}\left(\mathcal{S} \otimes_{\tau} \mathcal{T}\right) \otimes_{\text {min }} \mathcal{S}_{2}=\left(\mathcal{S} \otimes_{\tau} \mathcal{T}\right) \otimes_{c} \mathcal{S}_{2} \stackrel{(i d \otimes g) \otimes i d}{\longrightarrow} \mathcal{S} \otimes_{c} \mathcal{S}_{2}
$$

is a sequence of ucp maps such that their composition is the identity on $\mathcal{S} \otimes \mathcal{S}_{2}$. Since min $\leq$ c we obtain that $\mathcal{S}$ has property $\mathbb{S}_{2}$. The proof for $\mathcal{T}$ is similar.

The fact that $\mathcal{T}_{3} / J_{3}=\mathcal{S}_{2}$ together with Theorem 5.9 allow us characterize WEP as follows (we refer the reader to [11] for further applications of this result):

Theorem 10.10. A unital $C^{*}$-algebra $\mathcal{A}$ has $W E P$ if and only if the associated map $\mathcal{T}_{3} \otimes_{\text {min }}$ $\mathcal{A} \rightarrow\left(\mathcal{T}_{3} / J_{3}\right) \otimes_{\min } \mathcal{A}$ is a quotient map. In other words we have the complete order isomorphism

$$
\left(\mathcal{T}_{3} \otimes_{\min } \mathcal{A}\right) /\left(J_{3} \otimes \mathcal{A}\right)=\left(\mathcal{T}_{3} / J_{3}\right) \otimes_{\min } \mathcal{A}
$$

Proof. By using the projectivity of the maximal tensor product and $\mathrm{C}^{*}$-nuclearity of $T_{3}$ we have that

$$
\mathcal{S}_{2} \otimes_{\max } \mathcal{A}=\mathcal{T}_{3} / J_{3} \otimes_{\max } \mathcal{A}=\left(\mathcal{T}_{3} \otimes_{\max } \mathcal{A}\right) /\left(J_{3} \otimes \mathcal{A}\right)=\left(\mathcal{T}_{3} \otimes_{\min } \mathcal{A}\right) /\left(J_{3} \otimes \mathcal{A}\right)
$$

Now if $\mathcal{A}$ has WEP then it has property $\mathbb{S}_{2}$ and the equality in the theorem satisfies. Conversely if the equality is satisfied then $\mathcal{A}$ must have property $\mathbb{S}_{2}$, equivalently, WEP.

We now discuss some duality results from [10. Recall that we write $\mathcal{S}_{n}$ in the following basis form: $\mathcal{S}_{n}=\operatorname{span}\left\{g_{1}, \ldots, g_{n}, e, g_{1}^{*}, \ldots, g_{n}^{*}\right\}$. When we pass to dual basis we have that

$$
\mathcal{S}_{n}^{d}=\operatorname{span}\left\{\delta_{1}, \ldots, \delta_{n}, \delta, \delta_{1}^{*}, \ldots, \delta_{n}^{*}\right\} .
$$

We leave the elementary proof of the fact that $\delta_{g_{i}^{*}}=\delta_{i}^{*}$ to the reader. We also remind that $\delta$ is a faithful state and we consider it as the Archimedean matrix order unit for the dual operator system. We now see that $\mathcal{S}_{n}^{d}$ can be identified with an operator subsystem of $M_{2} \oplus M_{2} \oplus \cdots \oplus M_{2}$ (the direct sum of $n$ copies of $M_{2}$ ). To avoid the excessive notation we use the following:

$$
e=\left(I_{2}, \ldots, I_{2}\right), \quad e_{1}=\left(E_{12}, 0, \ldots, 0\right), \quad e_{2}=\left(0, E_{12}, 0, \ldots, 0\right), \ldots e_{n}=\left(0, \ldots, 0, E_{12}\right) .
$$

Consider the following map:

$$
\gamma: \mathcal{S}_{n}^{d} \rightarrow \oplus_{i=1}^{n} M_{2} \text { given by } \delta \mapsto e, \quad \delta_{i} \mapsto e_{i} \text { and } \delta_{i}^{*} \mapsto e_{i}^{*} \text { for } i=1, \ldots, n .
$$

Now we are ready to state: 
Theorem 10.11 (Farenick, Paulsen). The above map $\gamma: \mathcal{S}_{n}^{d} \rightarrow \oplus_{i=1}^{n} M_{2}$ is a unital complete order embedding.

By using the diagonal identification of $M_{2} \oplus M_{2}$ in $M_{4}$, in particular, we have that

$$
\mathcal{S}_{2}^{d}=\left\{\left(\begin{array}{cccc}
a & b & 0 & 0 \\
c & a & 0 & 0 \\
0 & 0 & a & d \\
0 & 0 & e & a
\end{array}\right) \quad: a, b, c, d, e \in \mathbb{C}\right\}
$$

In [43] it was shown by Wasserman that $C^{*}\left(F_{n}\right)$ is not exact for any $n \geq 2$. Clearly $\mathcal{S}_{n}$ contains enough unitaries in $C^{*}\left(F_{n}\right)$. The following is Corollary 9.6 in [21]:

Proposition 10.12. Suppose that $\mathcal{S} \subset \mathcal{A}$ contains enough unitaries. If $\mathcal{S}$ is exact then $\mathcal{A}$ is exact.

Corollary 10.13. $\mathcal{S}_{n}$ is not exact for any $n \geq 2$.

Exactness is stable under $\mathrm{C}^{*}$-algebra ideal quotients, that is, if a $\mathrm{C}^{*}$-algebra is exact then any of its quotient by an ideal has the same property (see [24] and [42]). This stability property is not valid for general operator system quotients even under the favorable conditions: The dimension of the operator system is finite and the kernel is a null subspace. In fact since $\mathcal{T}_{n}$ is $\mathrm{C}^{*}$-nuclear (i.e. (min,c)-nuclear) then in particular it is exact (equivalently (min,el)-nuclear). However, its quotient by the null subspace $J_{n}$, namely $\mathcal{S}_{n}=T_{n} / J_{n}$, is not exact.

Corollary 10.14. $M_{2} \oplus M_{2}$ (or $M_{4}$ ) has a five dimensional operator subsystem (namely $\mathcal{S}_{2}^{d}$ ) which does not possess the lifting property.

Proof. Since $\mathcal{S}_{2}$ is not exact then, by Theorem 6.6, its dual can not have the lifting property.

The following result is perhaps well known but we are unable to provide a reference.

Corollary 10.15. The Calkin algebra $\mathbb{B} / \mathbb{K}$ does not have WEP.

Proof. Assume for a contradiction that $\mathbb{B} / \mathbb{K}$ has WEP. This means that $\mathcal{S}_{2} \otimes_{\min } \mathbb{B} / \mathbb{K}=$ $\mathcal{S}_{2} \otimes_{c=\max } \mathbb{B} / \mathbb{K}$. Since $\mathcal{S}_{2}$ has the lifting property we also have that $\mathcal{S}_{2} \otimes_{\min } \mathbb{B}=\mathcal{S}_{2} \otimes_{\max } \mathbb{B}$. Thus,

$$
\mathcal{S}_{2} \otimes_{\max } \mathbb{B} / \mathbb{K}=\left(\mathcal{S}_{2} \otimes_{\max } \mathbb{B}\right) /\left(\mathcal{S}_{2} \otimes \mathbb{K}\right)=\left(\mathcal{S}_{2} \otimes_{\min } \mathbb{B}\right) /\left(\mathcal{S}_{2} \otimes \mathbb{K}\right)=\mathcal{S}_{2} \otimes_{\text {min }} \mathbb{B} / \mathbb{K} .
$$

This means that, by Theorem $7.2, \mathcal{S}_{2}$ is exact which is a contradiction.

Corollary 10.16. $\mathcal{S}_{2} \otimes_{\max } \mathcal{S}_{2}$ has the lifting property.

Proof. Note that $\left(\mathcal{S}_{2} \otimes_{\max } \mathcal{S}_{2}\right)^{d}=\mathcal{S}_{2}^{d} \otimes_{\min } \mathcal{S}_{2}^{d} \subset M_{4} \otimes_{\min } M_{4}$. Since exactness passes to operator subsystems we have that $\left(\mathcal{S}_{2} \otimes_{\max } \mathcal{S}_{2}\right)^{d}$ is exact. Thus, by Theorem 6.6, $\mathcal{S}_{2} \otimes_{\max } \mathcal{S}_{2}$ has the lifting property.

Remark: We don't know whether the lifting property is preserved under the maximal tensor product. For finite dimensional operator systems, by using Theorem 6.6 and 6.3, the same question can be reformulated as follows: Is exactness preserved under the minimal tensor product? If $\mathcal{S}$ and $\mathcal{T}$ contain enough unitaries in their enveloping $\mathrm{C}^{*}$-algebras (also under the assumption that both $\mathcal{S}$ and $\mathcal{T}$ are separable) the answer is affirmative. In fact, by Proposition 10.12, both $C_{e}^{*}(\mathcal{S})$ and $C_{e}^{*}(\mathcal{T})$ must be exact. Also note that both of these $\mathrm{C}^{*}$ algebras are separable. We know that every separable exact $\mathrm{C}^{*}$-algebra can be represented in a nuclear $\mathrm{C}^{*}$-algebra [26]. So $\mathcal{S}$ and $\mathcal{T}$ can be represented in nuclear $\mathrm{C}^{*}$-algebras, say $\mathcal{A}$ and $\mathcal{B}$, respectively. Note that $\mathcal{S} \otimes_{\min } \mathcal{T} \subset \mathcal{A} \otimes_{\min } \mathcal{B}$ and it is elementary to show that 
$\mathcal{A} \otimes_{\min } \mathcal{B}$ is again nuclear. Thus, $\mathcal{S} \otimes_{\min } \mathcal{T}$ embeds in a nuclear $\mathrm{C}^{*}$-algebra. Since nuclearity implies exactness and exactness passes to operator subsystems it follows that $\mathcal{S} \otimes_{\min } \mathcal{T}$ is exact. However, in general, the exactness of $\mathcal{S}$ may not pass to $C_{e}^{*}(\mathcal{S})$. In [27, Kirchberg and Wassermann construct a separable, (min,max)-nuclear operator system $\mathcal{S}$ with the property that $C_{u}^{*}(\mathcal{S})=C_{e}^{*}(\mathcal{S})$. Clearly $\mathcal{S}$ is exact. However, since $\operatorname{dim}(\mathcal{S}) \geq 3, C_{u}^{*}(\mathcal{S})$ equivalently $C_{e}^{*}(\mathcal{S})$, is not exact.

After these results we also relate the property $\mathbb{S}_{2}$ and the $\mathrm{KC}$.

Theorem 10.17. The following are equivalent:

(1) KC has an affirmative answer.

(2) $\mathcal{S}_{2}$ has property $\mathbb{S}_{2}$.

(3) Every finite dimensional operator system with the lifting property has property $\mathbb{S}_{2}$.

(4) If $\mathcal{S}$ is a finite dimensional exact operator system then $\mathcal{S}^{d}$ has property $\mathbb{S}_{2}$.

(5) If $\mathcal{S}$ is a finite dimensional $C^{*}$-nuclear operator system and $J$ is a null subspace of $\mathcal{S}$ then $\mathcal{S} / J$ has property $\mathbb{S}_{2}$.

(6) $\mathcal{S}_{2} \otimes_{\max } \mathcal{S}_{2}$ has property $\mathbb{S}_{2}$.

Proof. The equivalence of (1) and (2) is simply a restatement of Theorem 5.10. If we assume (1) then it follows that every finite dimensional operator system with lifting property has DCEP, in particular, property $\mathbb{S}_{2}$. This proves that (1) implies (3). Clearly (3) implies (6). If we assume (6) then Proposition 10.9 implies that $\mathcal{S}_{2}$ has property $\mathbb{S}_{2}$. Thus, $(2)$ is true. So we need to show that these are all equivalent to (4) and (5).

$(1) \Rightarrow(4)$ : Let $\mathcal{S}$ be an exact operator system. By Theorem 6.6, $\mathcal{S}^{d}$ has the lifting property and consequently, it has DCEP. DCEP implies property $\mathbb{S}_{2}$ thus (1) implies (4).

$(4) \Rightarrow(2)$ : In fact $\mathcal{S}_{2}^{d}$ is exact so its dual, namely $\mathcal{S}_{2}$, has property $\mathbb{S}_{2}$.

$(1) \Rightarrow(5)$ : If $\mathcal{S}$ is $\mathrm{C}^{*}$-nuclear, in particular, it has the lifting property. Thus, by Theorem 6.8, $\mathcal{S} / J$ has the lifting property. By using Theorem 5.10, $\mathcal{S} / J$ must have DCEP and, thus, it must have property $\mathbb{S}_{2}$.

$(5) \Rightarrow(2)$ : So, in particular, $T_{3} / J_{3}=\mathcal{S}_{2}$ has property $\mathbb{S}_{2}$. This finishes the proof.

In quantum mechanics, one of the basic problems in modeling an experiment is determining whether by using the classical probabilistic approach we can approximate all outcomes arising from the non-commutative setting. More precisely, Tsirelson's problem asks whether the nonrelativistic behaviors in a quantum experiment can be described by relativistic approach. The proper definitions and basic result in this question are beyond the scope of this paper and we refer the reader to [38], [20], [14]. In [20] it was shown that when the actors are Alice and Bob (that is, in the bipartite scenario) the question is reduced to whether

$$
C^{*}\left(\mathbb{F}_{\infty}\right) \otimes_{\min } C^{*}\left(\mathbb{F}_{\infty}\right)=C^{*}\left(\mathbb{F}_{\infty}\right) \otimes_{\max } C^{*}\left(\mathbb{F}_{\infty}\right),
$$

in other words, the Kirchberg Conjecture. When Charlie is also included, i.e. with three actors, Tsirelson's problem is known to be related to whether the minimal and the maximal tensor products of three copies of $C^{*}\left(\mathbb{F}_{\infty}\right)$ coincide. So we want to close this section with a discussion on this topic from an operator system perspective.

\section{Conjecture 10.18.}

$$
\bigotimes_{i=1}^{3}{ }_{\min } C^{*}\left(\mathbb{F}_{\infty}\right)=\bigotimes_{i=1}^{3} \max C^{*}\left(\mathbb{F}_{\infty}\right)
$$


This should be considered as an extended version of the Kirchberg Conjecture. An affirmative answer of Conjecture 10.18 implies that the Kirchberg Conjecture is true. In fact this follows from the fact that for any functorial tensor product $\tau$ and operator systems $\mathcal{S}$ and $\mathcal{T}$ we have that $\mathcal{S} \cong \mathcal{S} \otimes \mathbb{C} \subset \mathcal{S} \otimes_{\tau} \mathcal{T}$. So if we put $C=C^{*}\left(\mathbb{F}_{\infty}\right)$ then

$$
C \otimes_{\min } C \subset\left(C \otimes_{\min } C\right) \otimes_{\min } C \text { and } C \otimes_{\max } C \subset\left(C \otimes_{\max } C\right) \otimes_{\max } C .
$$

Thus, if Conjecture 10.18 is true then $\mathrm{KC}$ is also true. On the other hand even if we assume that $\mathrm{KC}$ has an affirmative answer it is still unknown whether Conjecture 10.18 is true or not. We want to start with the following observations which are perhaps well known and will be more convenient when we express this problem in terms of lower dimensional operator systems.

Theorem 10.19. The following are equivalent:

(1) Conjecture 10.18 has an affirmative answer.

(2) $C^{*}\left(\mathbb{F}_{\infty}\right) \otimes_{\max } C^{*}\left(\mathbb{F}_{\infty}\right)$ has WEP.

(3) We have that

$$
\bigotimes_{i=1}^{3}{ }_{\min } C^{*}\left(\mathbb{F}_{2}\right)=\bigotimes_{i=1}^{3} \max C^{*}\left(\mathbb{F}_{2}\right)
$$

(4) $C^{*}\left(\mathbb{F}_{2}\right) \otimes_{\max } C^{*}\left(\mathbb{F}_{2}\right)$ has WEP.

Proof. Since the identity on $C^{*}\left(\mathbb{F}_{\infty}\right)$ factors via ucp maps through $C^{*}\left(\mathbb{F}_{2}\right)$, by using the functoriality of the max tensor product, it follows that the identity on $C^{*}\left(\mathbb{F}_{\infty}\right) \otimes_{\max } C^{*}\left(\mathbb{F}_{\infty}\right)$ factors via ucp maps through $C^{*}\left(\mathbb{F}_{2}\right) \otimes_{\max } C^{*}\left(\mathbb{F}_{2}\right)$. So by using Lemma 5.2 we obtain that (4) implies (2). Since the identity on $C^{*}\left(\mathbb{F}_{2}\right)$ factors via ucp maps through $C^{*}\left(\mathbb{F}_{\infty}\right)$, we similarly obtain that (2) implies (4). The proof of the equivalence of (1) and (3) is based on the same fact. In general, if the identity on $\mathcal{S}$ decomposes into ucp maps through $\mathcal{T}$ (say $i d=\psi \circ \phi$ ), also assuming that $\mathcal{T} \otimes_{\text {min }} \mathcal{T} \otimes_{\text {min }} \mathcal{T}=\mathcal{T} \otimes_{\max } \mathcal{T} \otimes_{\text {max }} \mathcal{T}$, we have that the maps

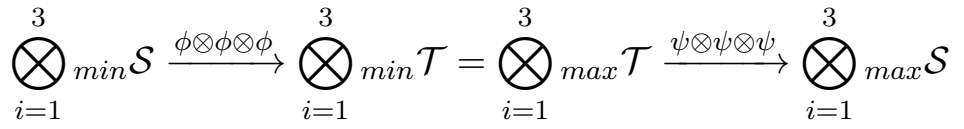

are ucp and their composition is the identity from triple minimal tensor product of $\mathcal{S}$ to maximal tensor product of $\mathcal{S}$. Thus these two tensor products coincide. This proves that (1) and (3) are equivalent. Now let $C$ stand for $C^{*}\left(\mathbb{F}_{\infty}\right)$. We will show that (2) implies (1). Since $C \otimes_{\max } C$ has WEP then in particular, by Lemma 5.2, this implies that $C$ has WEP, equivalently $C \otimes_{\min } C=C \otimes_{\max } C$. (Recall: These are all equivalent arguments in Kirchberg's theorem that we mentioned at the beginning of Section 5.) By using Kirchberg's WEP characterization we readily have that $\left(C \otimes_{\min } C\right) \otimes_{\min } C=\left(C \otimes_{\min } C\right) \otimes_{\max } C$. If we replace the min by max on the right hand side of this equation we obtain (1). Conversely suppose (1) is true. As we pointed out earlier, this, in particular, implies KC. Thus $C \otimes_{\min } C=C \otimes_{\max } C$. Since we assumed that the triple minimal and the maximal tensor product of $C$ coincide, by replacing a max by min (as seen below)

$$
\left(C \otimes_{\max } C\right) \otimes_{\max } C=\left(C \otimes_{\min =\max } C\right) \otimes_{\min } C
$$

we have that , $C \otimes_{\max } C$ satisfies Kirchberg's WEP characterization. So we obtain (2).

Theorem 10.20. The following implications hold:

$\mathcal{S}_{2} \otimes_{\min } \mathcal{S}_{2}$ has DCEP $\Rightarrow \mathcal{S}_{2} \otimes_{\min } \mathcal{S}_{2}$ has property $\mathbb{S}_{2} \Rightarrow$ Conjecture 10.18 is true.

Proof. Clearly DCEP implies property $\mathbb{S}_{2}$. Now, suppose that $\mathcal{S}_{2} \otimes_{\min } \mathcal{S}_{2}$ has property $\mathbb{S}_{2}$. It is not hard to see that $\mathcal{S}_{2} \otimes_{\text {min }} \mathcal{S}_{2}$ contains enough unitaries in $C^{*}\left(\mathbb{F}_{2}\right) \otimes_{\min } C^{*}\left(\mathbb{F}_{2}\right)$. We also remark that our assumption implies KC, that is, if $\mathcal{S}_{2} \otimes_{\min } \mathcal{S}_{2}$ has property $\mathbb{S}_{2}$ then, in 
particular, $\mathcal{S}_{2}$ has property $\mathbb{S}_{2}$ and by the above result $\mathrm{KC}$ has an affirmative answer. So we also have that $C^{*}\left(\mathbb{F}_{2}\right) \otimes_{\min } C^{*}\left(\mathbb{F}_{2}\right)=C^{*}\left(\mathbb{F}_{2}\right) \otimes_{\max } C^{*}\left(\mathbb{F}_{2}\right)$. Now by using Proposition 5.7, $\left(\mathcal{S}_{2} \otimes_{\min } \mathcal{S}_{2}\right) \otimes_{\min } \mathcal{S}_{2}=\left(\mathcal{S}_{2} \otimes_{\min } \mathcal{S}_{2}\right) \otimes_{c} \mathcal{S}_{2}$ implies that $\left(C^{*}\left(\mathbb{F}_{2}\right) \otimes_{\min } C^{*}\left(\mathbb{F}_{2}\right)\right) \otimes_{\min } C^{*}\left(\mathbb{F}_{2}\right)=$ $\left(C^{*}\left(\mathbb{F}_{2}\right) \otimes_{\min } C^{*}\left(\mathbb{F}_{2}\right)\right) \otimes_{\max } C^{*}\left(\mathbb{F}_{2}\right)$. Since the min on the right hand side can be replaced by max it follows that Conjecture 10.18 has an affirmative answer.

We don't know whether any of the converse implications in the above theorem hold or not.

Question 10.21. Is $\mathcal{S}_{2} \otimes_{c} \mathcal{S}_{2}=\mathcal{S}_{2} \otimes_{\max } \mathcal{S}_{2}$ ?

Question 10.22. Are DCEP or property $\mathbb{S}_{2}$ preserved under commuting tensor product? That is, if $\mathcal{S}$ and $\mathcal{T}$ are operator systems with DCEP (or having property $\mathbb{S}_{2}$ ) then does $\mathcal{S} \otimes_{c} \mathcal{T}$ have the same property?

An affirmative answer to any of these questions implies that $\mathrm{KC}$ is equivalent to Conjecture 10.18. We first remark that in the above theorem the min can be replaced by c, this follows from the fact that any of the arguments implies KC is true and, thus, $\mathcal{S}_{2} \otimes_{\min } \mathcal{S}_{2}=\mathcal{S}_{2} \otimes_{c} \mathcal{S}_{2}$. Now if we suppose that the first question has an affirmative answer then Theorem 10.17 (6) and the second argument in the above theorem gives this equivalence. Now suppose that the second question is true. If we suppose $\mathrm{KC}$ has an affirmative answer (so that $\mathcal{S}_{2}$ has DCEP) then $\mathcal{S}_{2} \otimes_{\min } \mathcal{S}_{2}=\mathcal{S}_{2} \otimes_{c} \mathcal{S}_{2}$ and this tensor product has DCEP (or property $\mathbb{S}_{2}$ ), thus, Conjecture 10.18 is also true.

In 30, Ozawa proved that $B(H) \otimes_{\min } B(H)$ does not have WEP where $H=l^{2}$. Since WEP and DCEP coincide for $\mathrm{C}^{*}$-algebras and $B\left(l^{2}\right)$ has WEP we see that DCEP, in general, does not preserved under the minimal tensor product.

Let $\mathcal{T}=\operatorname{span}\left\{I, E_{12}, E_{34}, E_{21}, E_{43}\right\} \subset M_{4}$. Recall that $\mathcal{S}_{2}^{d}$ and $\mathcal{T}$ are unitally completely order isomorphic. Thus we have that

$$
\mathcal{S}_{2} \otimes_{\text {min }} \mathcal{S}_{2}=\mathcal{S}_{2} \otimes_{\max } \mathcal{S}_{2} \Longleftrightarrow \mathcal{T} \otimes_{\min } \mathcal{T}=\mathcal{T} \otimes_{\max } \mathcal{T}
$$

which follows from the duality result in Theorem 6.3.

Question 10.23. Is $\mathcal{T} \otimes_{\min } \mathcal{T}=\mathcal{T} \otimes_{\max } \mathcal{T}$ ? Equivalently, is $\mathcal{S}_{2} \otimes_{\min } \mathcal{S}_{2}=\mathcal{S}_{2} \otimes_{\max } \mathcal{S}_{2}$ ?

Since $\mathrm{KC}$ is equivalent to the statement that $\mathcal{S}_{2} \otimes_{\min } \mathcal{S}_{2}=\mathcal{S}_{2} \otimes_{c} \mathcal{S}_{2}$ a positive answer to this question provides an affirmative answer to KC. In addition to this it also proves that Conjecture 10.18 is true since the condition in the Question 10.21 is satisfied and thus, by the previous paragraph, $\mathrm{KC}$ and Conjecture 10.18 are equivalent.

\section{Matricial Numerical Range of an Operator}

Let $\mathcal{S}$ be an operator system. For $x \in \mathcal{S}$ we define the $n^{\text {th }}$ matricial numerical range of $x$ by $w_{n}(x)=\left\{\varphi(x): \varphi: \mathcal{S} \rightarrow M_{n}\right.$ is ucp $\}$. Note that if we consider the operator subsystem $\mathcal{S}_{x}=\operatorname{span}\left\{e, x, x^{*}\right\}$ of $\mathcal{S}$ then, by using Arveson's extension theorem, the matricial ranges of $x$ remain same when it is considered as an element of $\mathcal{S}_{x}$. We also remark that if $T$ is an operator in $B(H)$ then its numerical range $W(T)=\{\langle T x, x\rangle:\|x\| \leq 1\}$ has the property that $\overline{W(T)}=w_{1}(T)$ (see [2], e.g.). For several properties and results regarding matricial ranges we refer the reader to [2], 35] and [39]. We include some of these results in the sequel. We start with the following well known fact (see [22, Lem. 4.1] e.g.).

Lemma 11.1. Let $\mathcal{S}$ be an operator system and $A \in M_{n}(\mathcal{S})$. Then $A$ is positive if and only if for every $k$ and for every ucp map $\varphi: \mathcal{S} \rightarrow M_{k}$ one has $\varphi^{(n)}(A)$ is positive in $M_{n}\left(M_{k}\right)$. 
This lemma indicates that the matricial ranges of an element $x$ in an operator system carry all the information of the operator subsystem $\mathcal{S}_{x}=\operatorname{span}\left\{e, x, x^{*}\right\}$ as $A \in M_{n}$ belongs to $w_{n}(x)$ if and only if there is a ucp map $\varphi: \mathcal{S}_{x} \rightarrow M_{n}$ such that $\varphi(x)=A$. Since $\varphi$ is ucp the image of any element in $\mathcal{S}_{x}$ can be determined by the value $\varphi(x)$. We can also state this as follows:

Proposition 11.2. Let $\mathcal{S}=\operatorname{span}\left\{e, x, x^{*}\right\}$ and $\mathcal{T}=\operatorname{span}\left\{e, y, y^{*}\right\}$ be two operator systems. Then the linear map $\varphi: \mathcal{S} \rightarrow \mathcal{T}$ given by $\varphi(e)=e, \varphi(x)=y$ and $\varphi\left(x^{*}\right)=y^{*}$, provided it is well-defined, is ucp if and only if $w_{n}(y) \subseteq w_{n}(x)$ for every $n$. Consequently, $\varphi$ is a complete order isomorphism if and only if $w_{n}(x)=w_{n}(y)$ for every $n$.

Proof. First suppose that $\varphi$ is ucp and let $A \in w_{n}(y)$. So there is ucp map $\psi: \mathcal{T} \rightarrow M_{n}$ such that $\psi(y)=A$. Clearly $\psi \circ \varphi$ is a ucp map from $\mathcal{S}$ into $M_{n}$ which maps $x$ to $A$. Thus, $A$ belongs to $w_{n}(x)$. Since $n$ was arbitrary this completes the proof of one direction. Now suppose that $w_{n}(y) \subseteq w_{n}(x)$ for every $n$. We will show that $\varphi$ is a cp map. The above lemma states that if $u$ is in $M_{n}(\mathcal{R})$, where $\mathcal{R}$ is any operator system, then $u$ is positive if and only if for every $k$ and for every ucp map $\phi: \mathcal{R} \rightarrow M_{k}$ one has $\phi^{(n)}(u)$ is positive. From this we deduce that an element of the form $u=e \otimes A+x \otimes B+x^{*} \otimes C$ in $M_{n}(\mathcal{S})$ is positive if and only if for every ucp map map $\phi: \mathcal{R} \rightarrow M_{k}$ one has $\phi^{(n)}(u)=I_{k} \otimes A+\varphi(x) \otimes B+\varphi(x)^{*} \otimes C$ is positive in $M_{k} \otimes M_{n}$ for every $k$, equivalently, $I_{k} \otimes A+X \otimes B+X^{*} \otimes C$ is positive in $M_{k} \otimes M_{n}$ for every $k$ and for every $X$ in $w_{k}(x)$. Of course, same property holds $M_{n}(\mathcal{T})$ in when $x$ is replaced by $y$. Now, by using the assumption $w_{k}(y) \subseteq w_{k}(x)$ for every $k$, it is easy to see that $\varphi$ is a cp map. The final part follows from the fact that $\varphi^{-1}$ is ucp if and only if $w_{n}(x) \subseteq w_{n}(y)$ for every $n$.

In this section we again use the notations $\mathbb{B}$ for $B\left(l^{2}\right)$ and $\mathbb{K}$ for the ideal of compact operators. A dot over an element will represents its image under the quotient map. We start with the following result given in [39].

Theorem 11.3 (Smith, Ward). Let $\dot{T} \in \mathbb{B} / \mathbb{K}$ and $n$ be an integer. Then there is a compact operator $K$ such that $w_{n}(T+K)=w_{n}(\dot{T})$.

Remark: In fact this theorem follows by using the $k$-lifting property of a finite dimensional operator system (Theorem 9.11). Moreover, we can deduce a more general form of this result: If $\mathcal{A}$ is a unital $\mathrm{C}^{*}$-algebra and $I \subset \mathcal{A}$ is an ideal then for any $\dot{a}$ in $\mathcal{A} / I$, and for any $k$ there is an element $x$ in $I$ such that $w_{k}(a+x)=w_{k}(\dot{a})$. This directly follows from the $k$-lifting property of the operator system $\mathcal{S}_{\dot{a}}=\left\{\dot{e}, \dot{a}, \dot{a}^{*}\right\}$ and the fact that every $k$-positive map defined from an operator system into $M_{k}$ is completely positive.

Turning back to the above result, we see that for a fixed $n$, an operator $T \in \mathbb{B}$ can be compactly perturbed such that the resulting operator and its its residue under the quotient map have the same $n^{t h}$ matricial range. Then the authors stated the following conjecture which is currently still open.

Smith Ward Problem (SWP): For every $T$ in $B(H)$ there is a compact operator $K$ such that $w_{n}(T+K)=w_{n}(\dot{T})$ for every $n$.

This question is also considered in [35] and several equivalent formulations have been given. In particular it was shown that it is enough to consider block diagonal operators, and for this case, the problem reduces to a certain distance question [35, Thm. 3.16]. However, the 
following remark which depends on an observation in [2] will be more relevant to us. We include the proof of this for the completeness of the paper.

Proposition 11.4 (Paulsen). The following are equivalent:

(1) SWP has an affirmative answer.

(2) For every operator subsystem of the form $\mathcal{S}_{\dot{T}}=\left\{\dot{I}, \dot{T}, \dot{T}^{*}\right\}$ in the Calkin algebra $\mathbb{B} / \mathbb{K}$, the inclusion $\mathcal{S}_{\dot{T}} \hookrightarrow \mathbb{B} / \mathbb{K}$ has a ucp lift on $\mathbb{B}$.

Proof. First suppose that $\mathcal{S}_{\dot{T}}$ has a ucp lift $\varphi$ on $\mathbb{B}$. Since $q(\varphi(\dot{T}))=T$, where $q$ is the quotient map from $\mathbb{B}$ into $\mathbb{B} / \mathbb{K}, \varphi(\dot{T})=T+K$ for some compact operator $K$. It is not hard to show that $w_{n}(T+K)=w_{n}(\dot{T})$ for every $n$. In fact, if $A \in w_{n}(\dot{T})$, say $A$ is the image $\phi(\dot{T})$ of some ucp map $\phi: \mathbb{B} / \mathbb{K} \rightarrow M_{n}$, then the composition $\phi \circ q$ is a ucp map from $\mathbb{B}$ into $M_{n}$ which maps $T$ to $A$. Conversely if $B$ is in $w_{n}(T)$, say $\psi(T)=B$ where $\psi: \mathbb{B} \rightarrow M_{n}$ is ucp, then $\psi \circ \varphi: \mathcal{S}_{\dot{T}} \rightarrow M_{n}$ is ucp that maps $\dot{T}$ to $B$. Since $T$ was arbitrary it follows that (1) is true. Conversely suppose that (1) holds. So for $\dot{T}$ in $\mathbb{B} / \mathbb{K}$ we can find $K$ in $\mathbb{K}$ such that $w_{n}(\dot{T})=w_{n}(T+K)$ for every $n$. Now, by using Proposition 11.2, $\mathcal{S}_{\dot{T}}$ and $\mathcal{S}_{T+K} \subset \mathbb{B}$ are unitally completely order isomorphic via $\dot{T} \mapsto T+K$. This map is ucp and a lift of the inclusion $\mathcal{S}_{\dot{T}} \hookrightarrow \mathbb{B} / \mathbb{K}$. So proof is done.

Depending on Proposition 7.4 and Theorem 6.6 we obtain the following formulations of the SWP:

Theorem 11.5. The following are equivalent:

(1) SWP has an affirmative answer.

(2) Every three dimensional operator system has the lifting property.

(3) Every three dimensional operator system is exact.

Proof. Equivalence of (2) and (3) follows from Theorem 6.6. If every three dimensional operator system is exact then then their duals, which covers all three dimensional operator systems, must have the lifting property and vice versa. Now suppose (2). This in particular implies that every operator subsystem of the form $\mathcal{S}_{\dot{T}}=\left\{\dot{I}, \dot{T}, \dot{T}^{*}\right\}$ in the Calkin algebra $\mathbb{B} / \mathbb{K}$, the inclusion $\mathcal{S}_{\dot{T}} \hookrightarrow \mathbb{B} / \mathbb{K}$ has a ucp lift on $\mathbb{B}$. Hence by the above result of Paulsen, we conclude that SWP has an affirmative answer. Now suppose (1) holds. Let $\mathcal{S}$ be a three dimensional operator system. We will show that $\mathcal{S}$ has the lifting property. Let $\varphi: \mathcal{S} \rightarrow \mathbb{B} / \mathbb{K}$ be a ucp map. Clearly the image $\varphi(\mathcal{S})$ is of the form $\mathcal{S}_{\dot{T}}=\left\{\dot{I}, \dot{T}, \dot{T}^{*}\right\}$ for some $T$ in $\mathbb{B}$. Since we assumed SWP, the above result of Paulsen ensures that $\mathcal{S}_{\dot{T}}$ has a ucp lift on $\mathbb{B}$, say $\psi$. Now $\psi \circ \varphi$ is a ucp lift of $\varphi$ on $\mathbb{B}$. Finally by using Proposition 7.4 we conclude that $\mathcal{S}$ has the lifting property.

Recall from Proposition 6.13 that every two dimensional operator system is $\mathrm{C}^{*}$-nuclear and consequently they are all exact and have the lifting property. On the other hand there is a five dimensional operator system, namely $\mathcal{S}_{2}$, which is not exact and, by Theorem 6.6. its dual $\mathcal{S}_{2}^{d}$, which embeds in $M_{2} \oplus M_{2}$, does not posses the lifting property.

Remark 11.6. There is a four dimensional operator system which is not exact and consequently, by Theorem 6.6, its dual does not have the lifting property.

Proof. It is well known that $\mathbb{F}_{2}$ embeds in $\mathbb{Z}_{2} * \mathbb{Z}_{3}$ (see [18, pg. 24] ,e.g.). So by using Proposition 8.8 of [41], $C^{*}\left(\mathbb{F}_{2}\right)$ embeds in $C^{*}\left(\mathbb{Z}_{2} * \mathbb{Z}_{3}\right)$ with a ucp inverse. Thus, the identity on $C^{*}\left(\mathbb{F}_{2}\right)$ decomposes via ucp maps through $C^{*}\left(\mathbb{Z}_{2} * \mathbb{Z}_{3}\right)$. This means that, by Lemma 5.2, any nuclearity property of $C^{*}\left(\mathbb{Z}_{2} * \mathbb{Z}_{3}\right)$ passes to $C^{*}\left(\mathbb{F}_{2}\right)$. Since $C^{*}\left(\mathbb{F}_{2}\right)$ is not exact we obtain that $C^{*}\left(\mathbb{Z}_{2} * \mathbb{Z}_{3}\right)$ cannot be exact. Note that $\mathbb{Z}_{2} * \mathbb{Z}_{3}$ can be described by $\left\langle a, b: a^{2}=b^{3}=e\right\rangle$ 
so, necessarily, a must be a self adjoint unitary in $C^{*}\left(\mathbb{Z}_{2} * \mathbb{Z}_{3}\right)$. Let $\mathcal{S}=\operatorname{span}\left\{e, a, b, b^{*}\right\} . \mathcal{S}$ is a four dimensional operator subsystem of $C^{*}\left(\mathbb{Z}_{3} * \mathbb{Z}_{2}\right)$ that contains enough unitaries. By Corollary 9.6 of [21] $\mathcal{S}$ cannot be exact. The remaining part follows from Theorem 6.6.

Now we turn back to the Kirchberg Conjecture (KC). Before we establish a connection between SWP and KC we recall that an operator system is (min,c)-nuclear if and only if it is $\mathrm{C}^{*}$-nuclear. We refer back to Section 4 for related discussion. We also remind the reader that $\mathrm{KC}$ is equivalent to the statement that every finite dimensional operator system that has the lifting property has the double commutant expectation property (DCEP). Now if we assume that both SWP and KC have affirmative answers then it follows that every operator system with dimension three is exact and has the lifting property, equivalently, they are all (min,el)nuclear and (min,er)-nuclear. Since we assumed KC it follows that all three dimensional operator systems must have DCEP, equivalently (el,c)-nuclearity. Finally, (min,el)-nuclearity and (el,c)-nuclearity implies (min,c)-nuclearity, that is, $\mathrm{C}^{*}$-nuclearity. Conversely if every operator system of dimension three is $\mathrm{C}^{*}$-nuclear this in particular implies they are all exact, (or have lifting property). Hence we obtain that

$$
\mathrm{KC}+\mathrm{SWP} \Longrightarrow \begin{gathered}
\text { every three dimensional } \\
\text { operator system is } \mathrm{C}^{*} \text {-nuclear }
\end{gathered} \Longrightarrow \mathrm{SWP}
$$

Consequently forming an example of a three dimensional operator system which is not $\mathrm{C}^{*}$ nuclear shows that both KC and SWP cannot be true. Showing indeed that they are all $\mathrm{C}^{*}$-nuclear provides an affirmative answer to SWP.

Question 11.7. We repeat a question we considered before: If $X$ is a compact subset of $\{z:|z| \leq 1\}$ then is $\mathcal{S}=\left\{1, z, z^{*}\right\}$, where $z$ is the coordinate function, $\mathrm{C}^{*}$-nuclear? When $X$ is the unit circle $\mathbb{T}$ then $\mathcal{S}$ coincides with $\mathcal{S}_{1}$ and for this case we know that $\mathcal{S}$ is $\mathrm{C}^{*}$-nuclear.

Acknowledgment: This paper is based on my Ph.D. Dissertation "Tensor Products of Operator Systems and Applications" under the supervision of Dr. Vern I. Paulsen. I would like to express my sincere gratitude to him for his guidance at every stage of this work.

\section{REFERENCES}

[1] W. B. Arveson, Subalgebras of $C^{*}$-algebras I, Acta Math. 123 (1969) 141-224.

[2] W. B. Arveson, Subalgebras of $C^{*}$-algebras II, Acta Math. 128 (1972) 271-308.

[3] D. P. Blecher and B. L. Duncan, Nuclearity-related properties for nonselfadjoint algebras, J. Operator Theory 65 (2011) 47-70.

[4] D. P. Blecher and V. I. Paulsen, Tensor products of operator spaces, J. Funct. Anal. 99 (1991), $262-292$.

[5] M. D. Choi, A Schwarz inequality for positive linear maps on $C^{*}$-algebras, Illinois J. Math. 18 (1974) 565-574.

[6] M. D. Choi and E. G. Effros, Injectivity and operator spaces, J. Funct. Anal. 24 (1977) 156-209.

[7] M. D. Choi and E. G. Effros, Nuclear $C^{*}$-algebras and the approximation property, Amer. J. Math. 100 (1978) 61-79.

[8] M. D. Choi and E. G. Effros, Nuclear $C^{*}$-algebras and injectivity: The general case, Indiana. Univ. Math. J. 26 (1977) 443-446.

[9] A. Connes, Classification of injective factors, cases $I I_{1}, I I_{\infty}, I I I_{\lambda}, \lambda \neq 1$, Ann. Math. 104 (1976) 73-116.

[10] D. Farenick and V. I. Paulsen, Operator system quotients of matrix algebras and their tensor products, arXiv:1101.0790.

[11] D. Farenick, A. S. Kavruk and V. I. Paulsen, $C^{*}$-algebras with the weak expectation property and a multivariable analogue of Ando's theorem on the numerical radius, arXiv:1107.0418.

[12] E. G. Effros, Injectives and tensor products for convex sets and $C^{*}$-algebras, NATO Advanced Study Institute, University College of Swansea, 1972.

[13] T. Fritz, Operator system structures on the unital direct sum of $C^{*}$-algebras, 2010, arXiv:1011.1247.

[14] T. Fritz, Tsirelson's problem and Kirchberg's conjecture, arXiv:1008.1168

[15] M. Hamana, Injective envelopes of operator systems, Publ. RIMS Kyote Univ. 15 (1979) 773-785. 
[16] K. H. Han, On maximal tensor products and quotient maps of operator systems, arXiv:1010.0380v2

[17] K. H. Han and V. Paulsen, An approximation theorem for nuclear operator systems, J. Funct. Anal. 261 (2011), 999-1009.

[18] P. de La Harpe, Topics in geometric group theory, The University of Chicago Press, (2000)

[19] N. Johnston, D. W. Kribs, V. I. Paulsen and R. Pereira, Minimal and maximal operator spaces and operator systems in entanglement theory arXiv:1010.1432.

[20] M. Junge, M. Navascues, C. Palazuelos, D. Perez-Garcia, V. B. Scholz and R. F. Werner, Connes' embedding problem and Tsirelson's problem, J. Math. Phys. 52, 012102 (2011)

[21] A. S. Kavruk, V. I. Paulsen, I. G. Todorov and M. Tomforde, Quotients, exactness and nuclearity in the operator system category, preprint.

[22] A. S. Kavruk, V. I. Paulsen, I. G. Todorov and M. Tomforde, Tensor products of operator systems, J. Funct. Anal., 261 (2011) 267-299.

[23] D. Kerr and H. Li On Gromov-Hausdorff convergence for operator metric spaces, J. Operator Theory, 62 (2009) 83-109.

[24] E. Kirchberg, On subalgebras of the CAR-algebra, J. Functional Anal. 129 (1995), 35-63.

[25] E. Kirchberg, On non-semisplit extensions, tensor products and exactness of group $C^{*}$-algebras, Invent. Math. 112 (1993) 449 - 489.

[26] E. Kirchberg and N.C Philips, Embedding of exact $C^{*}$-algebras in the Cuntz algebra $\mathcal{O}_{2}$, J. Reine Angew. Math. 525 (2000) 17-53.

[27] E. Kirchberg and S. Wassermann, $C^{*}$-algebras generated by operator systems, J. Funct. Anal., 155 (1998), $324-351$.

[28] C. Lance, Tensor products and nuclear $C^{*}$-algebras, Proceedings of Symposia in Pure Mathematics, Vol. 38 (1982) 379-399.

[29] C. Lance, On nuclear $C^{*}$-algebras, J. Funct. Anal. 12 (1973) 157-176.

[30] N. Ozawa, An application of expanders to $B\left(l^{2}\right) \otimes B\left(l^{2}\right)$, J. Funct. Anal. 198 (2003) 499-510.

[31] N. Ozawa, Local theory and local reflexivity for operator spaces, Ph.D. thesis, Texas A\&M Univ., 2000.

[32] N. Ozawa, On the lifting property for universal $C^{*}$-algebras of operator spaces, J. Operator Theory 46 (2001) 579-591.

[33] V. I. Paulsen, Completely bounded maps and operator algebras, Cambridge Studies in Advanced Mathematics 78, Cambridge University Press, 2002.

[34] V. I. Paulsen, The maximal operator space of a normed space, Proc. Edinburgh Math. Soc. (2) 39 (1996), no. 2, 309-323.

[35] V. I. Paulsen, Preservation of essential matrix ranges by compact perturbations, J. Operator Theory 8 (1982) 299-317.

[36] V.I. Paulsen, I.G. Todorov and M. Tomforde, Operator system structures on ordered spaces, preprint.

[37] V. I. Paulsen and M. Tomforde, Vector spaces with an order unit, Indiana Univ. Math. J., to appear.

[38] V. B. Scholz and R. F. Werner, Tsirelsons Problem, arXiv/0812.4305, 2008

[39] R. R. Smith and J. D. Ward, Matrix ranges for Hilbert space operators, Amer. J. Math., 102 (1980) 1031-1081.

[40] G. K. Pedersen, C*-Algebras and their automorphism groups, Academic Press, London, 1979.

[41] G. Pisier, Introduction to operator space theory, London Mathematical Society Lecture Note Series 294, Cambridge University Press, 2003.

[42] S. Wassermann, Subquotients of UHF C $C^{*}$-algebras, Math. Proc. Cambridge Phil. Soc. 115 (1994) $489-500$.

[43] S. Wassermann, The slice map problem for $C^{*}$-algebras, Proc. London Math. Soc. 32 (1976) 537-559.

[44] B. Xhabli, Universal operator systems on ordered spaces and their applications, Ph.D. thesis, University of Houston, 2009.

[45] B. Xhabli, The super operator system Structures and their applications in quantum entanglement theory, arXiv:1107.2366. 